\title{
Uracil Nucleosides with Reactive Group at C5 position: 5-(1-Halo-2- sulfonylvinyl)uridine Analogues
}

Yong Liang, Sazzad H. Suzol, Zhiwei Wen, Alain G. Artiles, Logesh Mathivathanan, Raphael G. Raptis and Stanislaw F. Wnuk*

Department of Chemistry and Biochemistry, Florida International University, Miami, Florida 33199, USA

wnuk@,fiu.edu

\section{SUPPORTING INFORMATION}

\section{CONTENTS}

1. Experimental Procedures and Characterization Data for Compounds: Pages S2-S10

2. Kinetics for the reactions between $\beta$-halovinylsulfone 19a-19c and PrSH: Page S11

3. Single-crystal X-ray crystallography for $\mathbf{1 9 a}$ and $\mathbf{2 4}$ :

Pages S12-S14

4. Additional references:

Page S15

5. ${ }^{1} \mathrm{H}$ and ${ }^{13} \mathrm{C}$ NMR Spectra for Compounds 3, 5, 6, 8, 9, 16a-b, 17a, 18a, 19a-d, 20a, 21a-c, 22-29: 
General Information. ${ }^{1} \mathrm{H}$ NMR spectra at $400 \mathrm{MHz}$ and ${ }^{13} \mathrm{C} \mathrm{NMR}$ at $100.6 \mathrm{MHz}$ were recorded in $\mathrm{CDCl}_{3}$ unless otherwise noted. All chemical shift values are reported in parts per million (ppm) and referenced to the residual solvent peaks $\left[\mathrm{CDCl}_{3}(7.26 \mathrm{ppm})\right.$ or DMSO- $d_{6}(2.54$ ppm)] for ${ }^{1} \mathrm{H}$ NMR and the $\mathrm{CDCl}_{3}$ (77.16 ppm) or DMSO- $d_{6}(39.52 \mathrm{ppm})$ for ${ }^{13} \mathrm{C}$ NMR spectra, with coupling constant $(J)$ values reported in Hz. HRMS were obtained in TOF (ESI) mode. TLC was performed on Merck kieselgel $60-\mathrm{F}_{254}$, and products were detected with $254 \mathrm{~nm}$ light. Merck kieselgel 60 (230-400 mesh) was used for column chromatography. Purity, yields and ratio of the products (crude and/or purified) were established via NMR with calibrated standards. All reagents and solvents were purchased from commercial suppliers and used without further purification. The 5-ethynyl substrates 3, 10-15 were prepared by standard methodology. ${ }^{\mathrm{S} 1,2}$

(E)-1- $\mathrm{N}$-benzyl-5-(1-chloro-2-tosylvinyl)uracil (3). Method A. The $\mathrm{FeCl}_{3} \cdot 6 \mathrm{H}_{2} \mathrm{O}(108 \mathrm{mg}$, $0.4 \mathrm{mmol})$, TBHP ( $82 \mu \mathrm{L}, 0.6 \mathrm{mmol} ; 70 \%$ TBHP in water) and $p$-toluenesulfonyl hydrazide 4 (74.5 mg, $0.4 \mathrm{mmol})$ was added to a stirring solution of 1- $N$-benzyl-5-ethynyluracil ${ }^{\mathrm{S} 3,4} \mathbf{1}(45.2 \mathrm{mg}$, $0.2 \mathrm{mmol})$ in $\mathrm{CH}_{3} \mathrm{CN}(2 \mathrm{~mL})$ at ambient temperature. The resulting solution was stirred at $80{ }^{\circ} \mathrm{C}$ (oil bath) for $4 \mathrm{~h}$. After cooling to ambient temperature, the volatiles were removed under the reduced pressure and the oily residue was column chromatographed (hexane/EtOAc, 1:1) to give 3 (71 mg, 85\%): ${ }^{1} \mathrm{H}$ NMR $\delta 2.23(\mathrm{~s}, 3 \mathrm{H}), 4.82(\mathrm{~s}, 2 \mathrm{H}), 6.70(\mathrm{~s}, 1 \mathrm{H}), 7.09(\mathrm{~d}, J=8.1 \mathrm{~Hz}, 2 \mathrm{H})$, 7.32-7.18 (m, 5H), $7.41(\mathrm{~d}, J=8.4 \mathrm{~Hz}, 2 \mathrm{H}), 7.42(\mathrm{~s}, 1 \mathrm{H}), 8.99(\mathrm{~s}, 1 \mathrm{H}) ;{ }^{13} \mathrm{C}$ NMR $\delta 21.8,52.4$, 108.9, 128.0, 128.7, 129.1, 129.4, 130.1, 134.2, 134.5, 136.7, 139.7, 145.4, 145.8, 150.1, 159.6; HRMS calcd for $\mathrm{C}_{20} \mathrm{H}_{18}{ }^{35} \mathrm{ClN}_{2} \mathrm{O}_{4} \mathrm{~S}[\mathrm{M}+\mathrm{H}]^{+} 417.0670$, found 417.0664 .

Alternative Procedure: The $\mathrm{Fe}(\mathrm{acac})_{3}(3 \mathrm{mg}, 0.01 \mathrm{mmol}), \mathrm{Ph}_{3} \mathrm{P}(3 \mathrm{mg}, 0.01 \mathrm{mmol})$ and tosyl chloride 2 (29 mg, $0.15 \mathrm{mmol})$ was added to a stirring solution of 1 (22.6 mg, $0.1 \mathrm{mmol})$ in toluene $(2 \mathrm{~mL})$ at ambient temperature. The resulting solution was stirred at $100{ }^{\circ} \mathrm{C}$ (oil bath) for $16 \mathrm{~h}$. After cooling to ambient temperature, the volatiles were removed under the reduced pressure and the oily residue was column chromatographed (hexane/EtOAc, 1:1) to give 3 (25 mg, $60 \%$ ) with the same spectroscopic data as described above.

(E)-1- $N$-benzyl-5-(1-bromo-2-tosylvinyl)uracil (5). Treatment of 1 - $N$-benzyl-5ethynyluracil 1 (45.2 mg, $0.2 \mathrm{mmol})$ with $p$-toluenesulfonyl hydrazide 4 (149.1 mg, $0.8 \mathrm{mmol})$ in the presence of $\mathrm{FeBr}_{3}(118.2 \mathrm{mg}, 0.4 \mathrm{mmol})$ by Method A gave 5 (56 mg, 61\%) and 1- $N$-benzyl5-(1,2-dibromovinyl)uracil 6 (26 mg, 33\%). Compound 5 had: ${ }^{1} \mathrm{H}$ NMR $\delta 2.40$ (s, 3H), 4.99 (s, 2H), $7.09(\mathrm{~s}, 1 \mathrm{H}), 7.27$ (d, $J=8.0 \mathrm{~Hz}, 2 \mathrm{H}), 7.40-7.45(\mathrm{~m}, 5 \mathrm{H}), 7.58(\mathrm{~s}, 1 \mathrm{H}), 7.58(\mathrm{~d}, J=8.2 \mathrm{~Hz}$, 2H), 9.29 (s, 1H); ${ }^{13} \mathrm{C}$ NMR $\delta 21.8,52.5,110.4,128.0,128.6,128.7,129.1,129.4,130.1,134.5$, 
136.6, 137.8, 145.0, 145.5, 149.9, 159.0. HRMS calcd for $\mathrm{C}_{20} \mathrm{H}_{17}{ }^{79} \mathrm{BrN}_{2} \mathrm{NaO}_{4} \mathrm{~S}[\mathrm{M}+\mathrm{Na}]^{+}$ 482.9985, found 482.9984 .

1-N-Benzyl-5-(1,2-dibromovinyl)uracil 6 had: ${ }^{1} \mathrm{H}$ NMR $\delta 4.69$ (s, 2H), 6.54 (s, 1H), 6.99 (s, 1H), 7.04-7.13 (m, 5H), 8.93 (s, 1H); ${ }^{13} \mathrm{C}$ NMR $\delta 51.9,109.0,112.1,112.5,128.3,129.0,129.4$, 134.6, 145.3, 150.4, 159.7. HRMS calcd for $\mathrm{C}_{13} \mathrm{H}_{10}{ }^{79} \mathrm{Br}_{2} \mathrm{~N}_{2} \mathrm{NaO}_{2}[\mathrm{M}+\mathrm{Na}]^{+} 406.9001$, found 406.9020 .

Method B. Sodium p-toluenesulfinate $7(71 \mathrm{mg}, 0.4 \mathrm{mmol})$ and NBS (36 $\mathrm{mg}, 0.2 \mathrm{mmol}$ ) were added to a stirring solution of $1(45.2 \mathrm{mg}, 0.2 \mathrm{mmol})$ in toluene $(2 \mathrm{~mL})$ at ambient temperature. The resulting solution was stirred at $80{ }^{\circ} \mathrm{C}$ (oil bath) for $5 \mathrm{~h}$. After cooling to ambient temperature, the volatiles were removed under the reduced pressure and the oily residue was chromatographed (hexane/EtOAc, 1:1) to give 5 (26 mg, 28\%).

(E)-1- $\mathrm{N}$-benzyl-5-(1-iodo-2-tosylvinyl)uracil (8). Treatment of $1(45.2 \mathrm{mg}, 0.2 \mathrm{mmol})$ with sodium $p$-toluenesulfinate $7(71 \mathrm{mg}, 0.4 \mathrm{mmol})$ in the presence of NIS (45 $\mathrm{mg}, 0.2 \mathrm{mmol})$ by Method B gave 8 (31 mg, 30\%) and 1- $N$-benzyl-5-(1,2-diiodovinyl)uracil 9 (9 mg, 9\%). Compound 8 had: ${ }^{1} \mathrm{H}$ NMR $\delta 2.35$ (s, 3H), 4.90 (s, 2H), 7.19 (d, $J=7.9$ Hz, 2H), 7.21 (s, 1H), 7.35-7.42 (m, 5H), $7.47(\mathrm{~s}, 1 \mathrm{H}), 7.48(\mathrm{~d}, J=8.5 \mathrm{~Hz}, 2 \mathrm{H}), 8.46(\mathrm{~s}, 1 \mathrm{H}) ;{ }^{13} \mathrm{C}$ NMR $\delta 21.8,52.5$, $101.6,113.0,128.0,128.7,129.1,129.4,130.2$, 134.5, 136.5, 143.2, 144.6, 145.4, 149.9, 159.1 . HRMS calcd for $\mathrm{C}_{20} \mathrm{H}_{17} \mathrm{IN}_{2} \mathrm{NaO}_{4} \mathrm{~S}[\mathrm{M}+\mathrm{Na}]^{+}$530.9846, found 530.9832 .

1-N-benzyl-5-(1,2-diiodovinyl)uracil 9 had: ${ }^{1} \mathrm{H}$ NMR $\delta 4.96$ (s, 2H), 7.30 (s, 1H), 7.33 (s, 1H), 7.34 - $7.42(\mathrm{~m}, 5 \mathrm{H}), 8.43(\mathrm{~s}, 1 \mathrm{H}) ;{ }^{13} \mathrm{C}$ NMR $\delta 51.9,85.2,87.2,117.5,128.4,129.0,129.4$, 134.6, 144.0, 150.1, 159.2. HRMS calcd for $\mathrm{C}_{13} \mathrm{H}_{10} \mathrm{I}_{2} \mathrm{~N}_{2} \mathrm{NaO}_{2}[\mathrm{M}+\mathrm{Na}]^{+}$502.8724, found 502.8749.

Method C. Iodine (25.3 mg, $0.1 \mathrm{mmol})$, TBHP (55 $\mu \mathrm{L}, 0.4 \mathrm{mmol} ; 70 \%$ TBHP in water) and p-toluenesulfonyl hydrazide $4(112 \mathrm{mg}, 0.6 \mathrm{mmol})$ were added to a stirring solution of $1(45.2 \mathrm{mg}$, $0.2 \mathrm{mmol})$ in $\mathrm{CH}_{3} \mathrm{CN}(2 \mathrm{~mL})$ at ambient temperature. The resulting solution was stirred at $80{ }^{\circ} \mathrm{C}$ (oil bath) for $4 \mathrm{~h}$. After cooling to ambient temperature, the volatiles were removed under the reduced pressure and the residue was chromatographed (hexane/EtOAc, 1:1) to give 8 (79 $\mathrm{mg}$, $78 \%$ ) with identical spectroscopic properties as described above.

(E)-2',3',5'-Tri-O-acetyl-5-(1-chloro-2-tosylvinyl)uridine (16a). Treatment of 2',3',5'-tri-Oacetyl-5-ethynyluridine $\mathbf{1 0}^{\mathrm{S} 5}(79 \mathrm{mg}, 0.2 \mathrm{mmol})$ with $p$-toluenesulfonyl hydrazide $4(52.2 \mathrm{mg}$, $0.28 \mathrm{mmol})$ in the presence of $\mathrm{FeCl}_{3} \cdot 6 \mathrm{H}_{2} \mathrm{O}(108 \mathrm{mg}, 0.4 \mathrm{mmol})$ by Method A gave 16a $(80 \mathrm{mg}$, 68\%): ${ }^{1} \mathrm{H}$ NMR $\delta 2.12$ (s, 3H), 2.14 (s, 3H), 2.18 (s, 3H), 2.42 (s, 3H), 4.37-4.47 (m, 3H), 5.36$5.41(\mathrm{~m}, 1 \mathrm{H}), 5.44(\mathrm{t}, J=5.5 \mathrm{~Hz}, 1 \mathrm{H}), 6.10(\mathrm{~d}, J=5.3 \mathrm{~Hz}, 1 \mathrm{H}), 6.94(\mathrm{~s}, 1 \mathrm{H}), 7.32$ (d, $J=8.1 \mathrm{~Hz}$, 2H), $7.71(\mathrm{~d}, J=8.2 \mathrm{~Hz}, 2 \mathrm{H}), 7.82(\mathrm{~s}, 1 \mathrm{H}), 8.63(\mathrm{~s}, 1 \mathrm{H}) ;{ }^{13} \mathrm{C}$ NMR $\delta 20.6,20.7,21.0,21.8,63.2$, 
70.4, 73.4, 80.7, 88.0, 109.4, 128.1, 130.2, 135.3, 136.7, 139.6, 141.6, 145.5, 149.2, 159.6, 169.6, 169.7, 170.5; HRMS calcd for $\mathrm{C}_{24} \mathrm{H}_{25}{ }^{35} \mathrm{ClN}_{2} \mathrm{NaO}_{11} \mathrm{~S}[\mathrm{M}+\mathrm{Na}]^{+} 607.0760$, found 607.0755.

(E)-2',3',5'-Tri-O-acetyl-5-(1-bromo-2-tosylvinyl)uracil (16b). Treatment of 10 (60 $\mathrm{mg}$, $0.15 \mathrm{mmol})$ with $p$-toluenesulfonyl hydrazide $4(90 \mathrm{mg}, 0.48 \mathrm{mmol})$ in the presence of $\mathrm{FeBr}_{3}(89$ $\mathrm{mg}, 0.3 \mathrm{mmol}$ ) by Method A gave 16b (46 mg, 53\%): ${ }^{1} \mathrm{H}$ NMR $\delta 2.12(\mathrm{~s}, 2 \mathrm{H}), 2.13(\mathrm{~s}, 3 \mathrm{H}), 2.18$ (s, 3H), $2.41(\mathrm{~s}, 3 \mathrm{H}), 4.36-4.4 .47(\mathrm{~m}, 3 \mathrm{H}), 5.38(\mathrm{t}, J=4.1 \mathrm{~Hz} 1 \mathrm{H}), 5.44(\mathrm{t}, J=5.4 \mathrm{~Hz} 1 \mathrm{H}), 6.1(\mathrm{~d}, J$ $=5.3 \mathrm{~Hz}, 1 \mathrm{H}), 7.15(\mathrm{~s}, 1 \mathrm{H}), 7.32(\mathrm{~d}, J=8.2 \mathrm{~Hz}, 2 \mathrm{H}), 7.7(\mathrm{~d}, J=8.3 \mathrm{~Hz}, 2 \mathrm{H}), 7.78(\mathrm{~s}, 1 \mathrm{H}), 8.96(\mathrm{~s}$, $1 \mathrm{H}) ;{ }^{13} \mathrm{C}$ NMR $\delta 20.5,20.7,21.0,21.8,63.2,70.4,73.4,81.6,88.0,110.8,128.1,128.4,130.2$, 136.5, 138.6, 140.7, 145.6, 149.2, 158.7, 169.6, 169.7, 170.4; HRMS calculated for $\mathrm{C}_{24} \mathrm{H}_{25}{ }^{81} \mathrm{BrN}_{2} \mathrm{NaO}_{11} \mathrm{~S}[\mathrm{M}+\mathrm{Na}]^{+}$653.0234, found 653.0216 .

Treatment of $10(60 \mathrm{mg}, 0.15 \mathrm{mmol})$ with $4(90 \mathrm{mg}, 0.48 \mathrm{mmol})$ in the presence of NBS (27 $\mathrm{mg}, 0.15 \mathrm{mmol}$ ) by Method B gave 16b (29 mg, 31\%) with identical spectroscopic data as described above.

$(E)-1-\left(2^{\prime}, 3^{\prime}, 5^{\prime}\right.$-Tri- $O$-acetyl- $\beta$-D-arabinofuranosyl)-5-(1-chloro-2-tosylvinyl)uracil (17a). Treatment of 1-(2',3',5'-tri- $O$-acetyl- $\beta$-D-arabinofuranosyl)-5-ethynyluracil ${ }^{\mathrm{S} 2,6} \mathbf{1 1}(80 \mathrm{mg}, 0.2$ mmol) with $p$-toluenesulfonyl hydrazide $4(52.2 \mathrm{mg}, 0.28 \mathrm{mmol})$ in the presence of $\mathrm{FeCl}_{3} \cdot 6 \mathrm{H}_{2} \mathrm{O}$ (108 mg, $0.4 \mathrm{mmol}$ ) by Method A (column chromatography; hexane/EtOAc, 4:6) gave 17a (77 mg, 66\%): ${ }^{1} \mathrm{H}$ NMR $\delta 2.11$ (s, 3H), 2.15 (s, 6H), 2.41 (s, 3H), 4.22-4.28 (m, 1H), 4.41 (dd, $J=$ 12.0, $4.2 \mathrm{~Hz}, 1 \mathrm{H}), 4.48$ (dd, $J=12.0,6.3 \mathrm{~Hz}, 1 \mathrm{H}), 5.13$ (dd, $J=2.7,1.3 \mathrm{~Hz}, 1 \mathrm{H}), 5.45$ (dd, $J=4.0$, $1.5 \mathrm{~Hz}, 1 \mathrm{H}), 6.30(\mathrm{~d}, J=4.0 \mathrm{~Hz}, 1 \mathrm{H}), 6.84(\mathrm{~s}, 1 \mathrm{H}), 7.33$ (d, $J=8.1 \mathrm{~Hz}, 2 \mathrm{H}), 7.75$ (d, $J=8.3 \mathrm{~Hz}$, 2H), $7.80(\mathrm{~s}, 1 \mathrm{H}), 9.36(\mathrm{~s}, 1 \mathrm{H}) ;{ }^{13} \mathrm{C}$ NMR $\delta 20.8,20.9,21.8,62.6,74.3,76.5,81.3,85.0,109.8$, 128.1, 130.2, 134.2, 136.7, 138.5, 141.5, 145.5, 149.1, 159.3, 169.2, 169.7, 170.8. HRMS calcd for $\mathrm{C}_{24} \mathrm{H}_{25}{ }^{35} \mathrm{ClN}_{2} \mathrm{NaO}_{11} \mathrm{~S}[\mathrm{M}+\mathrm{Na}]^{+}$607.0760, found 607.0760.

(E)-3',5'-Di-O-acetyl-5-(1-chloro-2-tosylvinyl)-2'-deoxyuridine (18a). Treatment of 3',5'di- $O$-acetyl-2'-deoxy-5-ethynyluridine $\mathbf{1 2}^{\mathrm{S} 1}(336 \mathrm{mg}, 1 \mathrm{mmol})$ with $p$-toluenesulfonyl hydrazide 4 (372 mg, $2 \mathrm{mmol}$ ) and $\mathrm{FeCl}_{3} \cdot 6 \mathrm{H}_{2} \mathrm{O}(540 \mathrm{mg}, 2 \mathrm{mmol})$ by Method A (column chromatography; hexane/EtOAc, $1: 1 \rightarrow 4: 6)$ gave 18a (400 mg, 76\%): ${ }^{1} \mathrm{H}$ NMR $\delta 2.12(\mathrm{~s}, 3 \mathrm{H}), 2.16(\mathrm{~s}, 3 \mathrm{H}), 2.27-$ $2.37(\mathrm{~m}, 1 \mathrm{H}), 2.42(\mathrm{~s}, 3 \mathrm{H}), 2.58(\mathrm{ddd}, J=1.7,5.5,14.3 \mathrm{~Hz}, 1 \mathrm{H}), 4.30-4.35$ (m, 2H), 4.46 (td, $J=$ 2.3, 5.0 Hz, 1H), 5.27 (d, $J=6.4 \mathrm{~Hz}, 1 \mathrm{H}), 6.35(\mathrm{dd}, J=5.5,8.5 \mathrm{~Hz}, 1 \mathrm{H}), 6.89$ (s, 1H), 7.32 (d, $J=$ $8.0 \mathrm{~Hz}, 1 \mathrm{H}), 7.69$ (d, $J=8.3 \mathrm{~Hz}, 1 \mathrm{H}), 7.88(\mathrm{~s}, 1 \mathrm{H}), 8.83(\mathrm{~s}, 1 \mathrm{H}) ;{ }^{13} \mathrm{C}$ NMR $\delta 21.02,21.04,21.8$, 38.2, 64.0, 74.4, 83.1, 85.7, 109.2, 127.9, 130.2, 134.7, 136.7, 139.9, 141.5, 145.6, 149.3, 158.9, 170.5, 170.6; HRMS calcd for $\mathrm{C}_{22} \mathrm{H}_{23}{ }^{35} \mathrm{ClN}_{2} \mathrm{NaO}_{9} \mathrm{~S}[\mathrm{M}+\mathrm{Na}]^{+} 549.0705$, found 549.0708. 
(E)-5-(1-Chloro-2-tosylvinyl)uridine (19a). Treatment of 5-ethynyluridine $\mathbf{1 3}^{\mathrm{S7}}$ (54 $\mathrm{mg}, 0.2$ mmol) with $p$-toluenesulfonyl hydrazide $4(80 \mathrm{mg}, 0.4 \mathrm{mmol})$ in the presence of $\mathrm{FeCl}_{3} \cdot 6 \mathrm{H}_{2} \mathrm{O}(108$ mg, $0.4 \mathrm{mmol}$ ) by Method A (column chromatography; $\mathrm{CHCl}_{3} / \mathrm{MeOH}$; 95:5 $\rightarrow$ 90:10) gave 19a (54 mg, 59\%): UV (MeOH) $\lambda_{\max } 242,276 \mathrm{~nm}$ ( $\varepsilon$ 14100, 10800), $\lambda_{\min } 230,261 \mathrm{~nm}(\varepsilon 13200$, 10200); ${ }^{1} \mathrm{H}$ NMR (DMSO- $\left.d_{6}\right) \delta 2.41(\mathrm{~s}, 3 \mathrm{H}), 3.58(\mathrm{ddd}, J=12.0,5.0,3.1 \mathrm{~Hz}, 1 \mathrm{H}), 3.67$ (ddd, $J=$ $11.9,4.9,3.1 \mathrm{~Hz}, 1 \mathrm{H}), 3.90(\mathrm{q}, J=3.2 \mathrm{~Hz}, 1 \mathrm{H}), 4.01(\mathrm{q}, J=4.9 \mathrm{~Hz}, 1 \mathrm{H}), 4.07$ (q, $J=5.0 \mathrm{~Hz}, 1 \mathrm{H})$, $5.14(\mathrm{~d}, J=5.0 \mathrm{~Hz}, 1 \mathrm{H}), 5.47(\mathrm{~d}, J=5.6 \mathrm{~Hz}, 1 \mathrm{H}), 5.80(\mathrm{~d}, J=4.8 \mathrm{~Hz}, 1 \mathrm{H}), 7.42(\mathrm{~d}, J=8.1 \mathrm{~Hz}$, $2 \mathrm{H}), 7.53(\mathrm{~s}, 1 \mathrm{H}), 7.57-7.79(\mathrm{~m}, 2 \mathrm{H}), 8.25(\mathrm{~s}, 1 \mathrm{H}), 11.67(\mathrm{~s}, 1 \mathrm{H}) ;{ }^{13} \mathrm{C}$ NMR (DMSO-d $\left.d_{6}\right) \delta 21.1$, 60.5, 69.5, 74.1, 79.1, 85.0, 88.5, 108.8, 127.6, 129.8, 134.0, 136.9, 139.6, 141.5, 144.8, 149.8, 159.4; HRMS calcd for: $\mathrm{C}_{18} \mathrm{H}_{20}{ }^{35} \mathrm{ClN}_{2} \mathrm{O}_{8} \mathrm{~S}[\mathrm{M}+\mathrm{H}]^{+}$for 459.0623 , found 459.0620 .

(E)-5-(1-Bromo-2-tosylvinyl)uridine (19b). Treatment of 13 (54 mg, $0.2 \mathrm{mmol})$ with $p$ toluenesulfonyl hydrazide $4(80 \mathrm{mg}, 0.4 \mathrm{mmol})$ in the presence of NBS (36 $\mathrm{mg}, 0.2 \mathrm{mmol})$ by Method A (column chromatography; $\mathrm{CHCl}_{3} / \mathrm{MeOH}, 95: 5 \rightarrow$ 90:10) gave 19b (41.3 mg, 41\%): UV (MeOH) $\lambda_{\max } 239,277 \mathrm{~nm}(\varepsilon 13500,10200), \lambda_{\min } 232,264 \mathrm{~nm}(\varepsilon 12800,9600) ;{ }^{1} \mathrm{H}$ NMR (DMSO- $\left.d_{6}\right) \delta 2.4(\mathrm{~s}, 3 \mathrm{H}), 3.54-3.72(\mathrm{~m}, 2 \mathrm{H}), 3.88-3.93(\mathrm{~m}, 1 \mathrm{H}), 3.96-4.1(\mathrm{~m}, 2 \mathrm{H}), 5.1-5.2(\mathrm{~m}, 2 \mathrm{H})$, $5.48(\mathrm{~d}, J=5.6 \mathrm{~Hz}, 1 \mathrm{H}), 5.8(\mathrm{~d}, J=4.6 \mathrm{~Hz}, 1 \mathrm{H}), 7.43(\mathrm{~d}, J=7.9 \mathrm{~Hz}, 2 \mathrm{H}), 7.65(\mathrm{~S}, 1 \mathrm{H}), 7.68(\mathrm{~d}, J$ $=7.5 \mathrm{~Hz}, 2 \mathrm{H}), 8.22(\mathrm{~s}, 1 \mathrm{H}), 11.68(\mathrm{~s}, 1 \mathrm{H}) ;{ }^{13} \mathrm{C} \mathrm{NMR}\left(\mathrm{DMSO}-d_{6}\right) \delta 21.1,60.5,69.6,74.2,85.0$, $88.5,110.4,127.7,129.3,129.8,136.7,136.9,140.7,144.8,149.7,159.3$; HRMS calcd for $\mathrm{C}_{18} \mathrm{H}_{19}{ }^{81} \mathrm{BrN}_{2} \mathrm{NaO}_{8} \mathrm{~S}[\mathrm{M}+\mathrm{Na}]^{+}$526.9917, found 526.9916.

(E)-5-(1-Iodo-2-tosylvinyl)uridine (19c). Treatment of 13 (26.8 $\mathrm{mg}, 0.1 \mathrm{mmol})$ with sodium p-toluenesulfinate 7 (35.6 mg, $0.2 \mathrm{mmol})$ in the presence of NIS (23 mg, $0.1 \mathrm{mmol}$ ) by Method B (column chromatography; $\mathrm{CHCl}_{3} / \mathrm{MeOH} ; 95: 5 \rightarrow$ 90:10) gave 19c $(21.5 \mathrm{mg}, 42 \%): \mathrm{UV}(\mathrm{MeOH})$ $\lambda_{\max } 235,262,291$ (sh) nm (E 14150, 12650), $\lambda_{\min } 220,252 \mathrm{~nm}(\varepsilon 13100,12400) ;{ }^{1} \mathrm{H}$ NMR $\left(\mathrm{DMSO}-d_{6}\right) \delta 2.4(\mathrm{~s}, 3 \mathrm{H}), 3.54-3.70(\mathrm{~m}, 2 \mathrm{H}), 3.87-3.92(\mathrm{~m}, 1 \mathrm{H}), 3.97-4.09(\mathrm{~m}, 2 \mathrm{H}), 5.8(\mathrm{~d}, J=$ $4.9 \mathrm{~Hz}, 1 \mathrm{H}), 7.4(\mathrm{~d}, J=8.1 \mathrm{~Hz}, 2 \mathrm{H}), 7.65$ (d, $J=8.3 \mathrm{~Hz}, 2 \mathrm{H}), 7.72(\mathrm{~s}, 1 \mathrm{H}), 8.07$ (s, 1H), 11.6 (s, $1 \mathrm{H}$ ); ${ }^{13} \mathrm{C}$ NMR (DMSO- $d_{6}$ ) $\delta 21.1,60.7,69.7,74.1,85.1,88.4,99.5,111.4,127.6,129.8,136.7$, 138.3, 142.6, 144.6, 149.8, 159.2; HRMS calculated for $\mathrm{C}_{18} \mathrm{H}_{19} \mathrm{IN}_{2} \mathrm{NaO}_{8} \mathrm{~S}[\mathrm{M}+\mathrm{Na}]^{+}$572.9799, found 572.9798 .

Treatment of $13(26.8 \mathrm{mg}, 0.1 \mathrm{mmol})$ with iodine $(14 \mathrm{mg}, 0.055 \mathrm{mmol})$ and $4(56 \mathrm{mg}, 0.3$ mmol) by Method C gave 19c (11 mg, 20\%) with the spectroscopic data as described above.

(E)-5-(2-Tosylvinyl)uridine (19d). Step $a$. The $p$-toluenesulfonic acid (62 $\mathrm{mg}, 0.4 \mathrm{mmol})$ was added to a solution of 2',3',5'-tri- $O$-acetyl-5-ethynyluridine $10(68 \mathrm{mg}, 0.17 \mathrm{mmol})$ in dry THF $(3 \mathrm{~mL})$ containing $\mathrm{Cu}(\mathrm{OAc})_{2} \cdot 2 \mathrm{H}_{2} \mathrm{O}(3 \mathrm{mg}, 0.015 \mathrm{mmol})$ under nitrogen at ambient 
temperature. The resulting solution was stirred at $60{ }^{\circ} \mathrm{C}$ (oil bath) for $7 \mathrm{~h}$. After cooling to ambient temperature, the volatiles were evaporated under the reduced pressure and the residue was column chromatographed (hexane/EtOAc, $1: 1 \rightarrow 4: 6)$ to give (E)-2',3',5'-tri-O-acetyl-5-(2tosylvinyl)uridine (31.5 mg, 35\%): ${ }^{1} \mathrm{H}$ NMR $\delta 2.10(\mathrm{~s}, 3 \mathrm{H}), 2.12(\mathrm{~s}, 2 \mathrm{H}), 2.21(\mathrm{~s}, 3 \mathrm{H}), 2.42(\mathrm{~s}, 3 \mathrm{H})$, $4.30-4.51(\mathrm{~m}, 3 \mathrm{H}), 5.31(\mathrm{t}, J=5.3 \mathrm{~Hz}, 1 \mathrm{H}), 5.36(\mathrm{t}, J=5.2 \mathrm{~Hz}, 1 \mathrm{H}), 6.00(\mathrm{~d}, J=4.8 \mathrm{~Hz}, 1 \mathrm{H})$, $7.19(\mathrm{~d}, J=15.0 \mathrm{~Hz}, 1 \mathrm{H}), 7.32(\mathrm{~d}, J=8.0 \mathrm{~Hz}, 2 \mathrm{H}), 7.62$ (d, $J=15.0 \mathrm{~Hz}, 1 \mathrm{H}), 7.76$ (d, $J=8.2 \mathrm{~Hz}$, $2 \mathrm{H}), 7.79(\mathrm{~s}, 1 \mathrm{H}), 8.70(\mathrm{~s}, 1 \mathrm{H})$. Step $b$. Methanolic ammonia $(1.5 \mathrm{~mL})$ was added to the solution of the material from step a $(25.5 \mathrm{mg}, 0.045 \mathrm{mmol})$ in $\mathrm{MeOH}(1.5 \mathrm{~mL})$ at $0{ }^{\circ} \mathrm{C}$ and the resulting solution was stirred for overnight. Volatiles were evaporated and the residue was column chromatographed $\left(\mathrm{CHCl}_{3} / \mathrm{MeOH}, 9: 1\right)$ to give 19d (12 mg, 63\%): ${ }^{1} \mathrm{H}$ NMR (MeOD- $\left.d_{4}\right) \delta 2.42$ (s, $3 \mathrm{H}), 3.77(\mathrm{dd}, J=12.4,2.6 \mathrm{~Hz}, 1 \mathrm{H}), 3.93(\mathrm{dd}, J=12.4,2.6 \mathrm{~Hz}, 1 \mathrm{H}), 4.02(\mathrm{dt}, J=5.2,2.6 \mathrm{~Hz}, 1 \mathrm{H})$, $4.12-4.21(\mathrm{~m}, 2 \mathrm{H}), 5.87(\mathrm{~d}, J=3.1 \mathrm{~Hz}, 1 \mathrm{H}), 7.30(\mathrm{~d}, J=15.0 \mathrm{~Hz}, 1 \mathrm{H}), 7.39(\mathrm{~d}, J=8.0 \mathrm{~Hz}, 2 \mathrm{H})$, $7.48(\mathrm{~d}, J=15.0 \mathrm{~Hz}, 1 \mathrm{H}), 7.74(\mathrm{~d}, J=8.4 \mathrm{~Hz}, 2 \mathrm{H}), 8.61(\mathrm{~s}, 1 \mathrm{H}) ;{ }^{13} \mathrm{C} \mathrm{NMR}\left(\mathrm{MeOD}-d_{4}\right) \delta 21.5$, 61.5, 70.4, 76.2, 86.2, 91.3, 108.7, 128.2, 128.5, 131.1, 136.2, 139.6, 145.8, 146.6, 151.1, 163.3. HRMS calculated for $\mathrm{C}_{18} \mathrm{H}_{20} \mathrm{~N}_{2} \mathrm{NaO}_{8} \mathrm{~S}[\mathrm{M}+\mathrm{Na}]^{+} 447.0833$, found 447.0802.

(E)-1-( $\beta$-D-Arabinofuranosyl)-5-(1-chloro-2-tosylvinyl)uracil (20a). Treatment of 1-( $\beta$-Darabinofuranosyl)-5-ethynyluracil ${ }^{\mathrm{S} 8} 14(27 \mathrm{mg}, 0.1 \mathrm{mmol})$ with $p$-toluenesulfonyl hydrazide 4 (40 $\mathrm{mg}, 0.2 \mathrm{mmol}$ ) in the presence of $\mathrm{FeCl}_{3} \cdot 6 \mathrm{H}_{2} \mathrm{O}(54 \mathrm{mg}, 0.2 \mathrm{mmol})$ by Method A (column chromatography; $\left.\mathrm{CHCl}_{3} / \mathrm{MeOH}, 95: 5 \rightarrow 90: 10\right)$ gave 20a $(28 \mathrm{mg}, 61 \%): \mathrm{UV}(\mathrm{MeOH}) \lambda_{\max } 241$, $282 \mathrm{~nm}(\varepsilon 12900,9800), \lambda_{\min } 262 \mathrm{~nm} \varepsilon$ 9700); ${ }^{1} \mathrm{H}$ NMR $\left(\mathrm{MeOH}-d_{4}\right) \delta 2.44(\mathrm{~s}, 3 \mathrm{H}), 3.76-3.93(\mathrm{~m}$, 2H), $3.98(\mathrm{dt}, J=4.9,3.8 \mathrm{~Hz}, 1 \mathrm{H}), 4.16(\mathrm{t}, J=3.5 \mathrm{~Hz}, 1 \mathrm{H}), 4.21(\mathrm{dd}, J=4.3,3.1 \mathrm{~Hz}, 1 \mathrm{H}), 6.17$ (d, $J=4.3 \mathrm{~Hz}, 1 \mathrm{H}), 7.22(\mathrm{~s}, 1 \mathrm{H}), 7.31-7.45(\mathrm{~m}, 2 \mathrm{H}), 7.66-7.81(\mathrm{~m}, 2 \mathrm{H}), 8.07$ (s, 1H); ${ }^{13} \mathrm{C}$ NMR $(100$ MHz, DMSO- $\left.d_{6}\right) \delta 21.1,60.5,75.1,75.2,85.1,85.7,107.7,127.8,129.8,133.7,136.9,139.7$, 142.1, 144.7, 149.5, 159.6; HRMS calcd for: $\mathrm{C}_{18} \mathrm{H}_{19}{ }^{35} \mathrm{ClN}_{2} \mathrm{NaO}_{8} \mathrm{~S}[\mathrm{M}+\mathrm{Na}]^{+}$for 481.0443, found 481.0443 .

(E)-5-(1-Chloro-2-tosylvinyl)-2'-deoxyuridine (21a). Treatment of 5-ethynyl-2'deoxyuridine ${ }^{1,7} 15$ (50.5 mg, $\left.0.2 \mathrm{mmol}\right)$ with $p$-toluenesulfonyl hydrazide 4 (112 mg, $0.6 \mathrm{mmol}$ ) in the presence of $\mathrm{FeCl}_{3} \cdot 6 \mathrm{H}_{2} \mathrm{O}(108 \mathrm{mg}, 0.4 \mathrm{mmol})$ and TBHP $(0.11 \mathrm{~mL}, 0.8 \mathrm{mmol} ; 70 \%$ TBHP in water) by Method A (column chromatography; $\left.\mathrm{CHCl}_{3} / \mathrm{MeOH} ; 9: 1 \rightarrow 85: 15\right)$ gave 21a (80 mg, 90\%) as white solid: UV (MeOH) $\left.\lambda_{\max } 241,276 \mathrm{~nm}(\varepsilon 13100,10150), \lambda_{\min } 262 \mathrm{~nm} \varepsilon 9600\right) ;{ }^{1} \mathrm{H}$ NMR (MeOD- $\left.d_{4}\right) \delta 2.25-2.34(\mathrm{~m}, 1 \mathrm{H}), 2.38(\mathrm{ddd}, J=13.4,6.0,4.1 \mathrm{~Hz}, 1 \mathrm{H}), 2.45(\mathrm{~s}, 3 \mathrm{H}), 3.78$ (dd, $J=12.0,3.5 \mathrm{~Hz}, 1 \mathrm{H}), 3.84(\mathrm{dd}, J=12.0,2.9 \mathrm{~Hz}, 1 \mathrm{H}), 3.99$ (dd, $J=6.3,3.0 \mathrm{~Hz}, 1 \mathrm{H}), 4.37-$ 4.53(m, 1H), 6.31 (t, $J=6.4 \mathrm{~Hz}, 1 \mathrm{H}), 7.23(\mathrm{~s}, 1 \mathrm{H}), 7.39$ (d, $J=7.8 \mathrm{~Hz}, 2 \mathrm{H}), 7.69$ (d, $J=7.8 \mathrm{~Hz}$, 
2H), $8.31(\mathrm{~s}, 1 \mathrm{H}) ;{ }^{13} \mathrm{C}$ NMR (MeOD- $\left.d_{4}\right) \delta 21.6,41.8,62.7,72.0,87.0,89.3,109.7,129.1,131.0$, 136.0, 138.3, 142.0, 143.9, 146.8, 151.3, 161.6; HRMS calcd for $\mathrm{C}_{18} \mathrm{H}_{19}{ }^{35} \mathrm{ClN}_{2} \mathrm{NaO}_{7} \mathrm{~S}[\mathrm{M}+\mathrm{Na}]^{+}$ 465.0494 , found 465.0493 .

(E)-5-(1-Bromo-2-tosylvinyl)-2'-deoxyuridine (21b). Treatment of 15 (50 $\mathrm{mg}, 0.2 \mathrm{mmol})$ with $p$-toluenesulfonyl hydrazide 4 ( $80 \mathrm{mg}, 0.4 \mathrm{mmol})$ in the presence of NBS (36 $\mathrm{mg}, 0.2 \mathrm{mmol})$ by Method $\mathrm{C}$ (column chromatography; $\mathrm{CHCl}_{3} / \mathrm{MeOH}, 95: 5$ ) gave $21 \mathbf{b}$ (39 mg, 44\%). UV $(\mathrm{MeOH}) \lambda_{\max } 236,276 \mathrm{~nm}\left(\varepsilon\right.$ 14500, 10500), $\lambda_{\min } 265 \mathrm{~nm}(\varepsilon 10350) ;{ }^{1} \mathrm{H}$ NMR (DMSO- $\left.d_{6}\right) \delta$ 2.07-2.16 (m, 1H), $2.20(\mathrm{ddd}, J=3.5,6.0,13.2 \mathrm{~Hz}, 1 \mathrm{H}), 2.40$ (s, 3H), 3.57 (dd, $J=3.7,11.9 \mathrm{~Hz}$, $2 \mathrm{H}), 3.62(\mathrm{dd}, J=3.5,11.9 \mathrm{~Hz}, 1 \mathrm{H}), 3.84(\mathrm{dd}, J=3.2,6.5 \mathrm{~Hz}, 1 \mathrm{H}), 4.27(\mathrm{dd}, J=2.9,5.7 \mathrm{~Hz}, 1 \mathrm{H})$, $6.17(\mathrm{t}, J=6.6 \mathrm{~Hz}, 1 \mathrm{H}), 7.42(\mathrm{~d}, J=8.0 \mathrm{~Hz}, 2 \mathrm{H}), 7.66$ (d, $J=7.3 \mathrm{~Hz}, 2 \mathrm{H}), 7.67(\mathrm{~s}, 3 \mathrm{H}), 8.16$ (s, $1 \mathrm{H}), 11.66(\mathrm{~s}, 1 \mathrm{H}) ;{ }^{13} \mathrm{C}$ NMR (DMSO- $\left.d_{6}\right) \delta 21.1,40.2,61.1,70.2,84.9,87.8,110.2,127.6,129.9$, $136.77,136.82,140.6,144.9,149.5,159.3$; HRMS calcd for $\mathrm{C}_{18} \mathrm{H}_{20}{ }^{81} \mathrm{BrN}_{2} \mathrm{O}_{7} \mathrm{~S}[\mathrm{M}+\mathrm{H}]^{+}$ 487.0169 , found 487.0168 .

(E)-5-(1-Iodo-2-tosylvinyl)-2'-deoxyuridine (21c). Treatment of 15 (50 mg, $0.2 \mathrm{mmol})$ with sodium $p$-toluenesulfinate $7(71 \mathrm{mg}, 0.4 \mathrm{mmol})$ in the presence of NIS (45 $\mathrm{mg}, 0.2 \mathrm{mmol})$ by Method B (column chromatography; $\left.\mathrm{CHCl}_{3} / \mathrm{MeOH}, 95: 5\right)$ gave 21c (32 mg, 30\%): UV (MeOH) $\lambda_{\max } 233,263,287(\mathrm{sh}) \mathrm{nm}(\varepsilon 14200,12650), \lambda_{\min } 221,252 \mathrm{~nm}(\varepsilon 13400,12300) ;{ }^{1} \mathrm{H}$ NMR $\left(\mathrm{DMSO}-d_{6}\right) \delta 2.10(\mathrm{ddd}, J=13.2,7.2,5.8 \mathrm{~Hz}, 1 \mathrm{H}), 2.19(\mathrm{dt}, J=13.3,4.5 \mathrm{~Hz}, 1 \mathrm{H}), 2.40(\mathrm{~s}, 3 \mathrm{H})$, 3.52-3.71 (m, 2H), $3.83(\mathrm{t}, J=3.4 \mathrm{~Hz}, 1 \mathrm{H}), 4.26(\mathrm{~d}, J=5.2 \mathrm{~Hz}, 1 \mathrm{H}), 5.05(\mathrm{t}, J=5.0 \mathrm{~Hz}, 1 \mathrm{H}), 5.30$ $(\mathrm{d}, J=4.2 \mathrm{~Hz}, 1 \mathrm{H}), 6.16(\mathrm{t}, J=6.6 \mathrm{~Hz}, 1 \mathrm{H}), 7.41(\mathrm{~d}, J=8.0 \mathrm{~Hz}, 2 \mathrm{H}), 7.64(\mathrm{~d}, J=8.4 \mathrm{~Hz}, 2 \mathrm{H})$, $7.72(\mathrm{~s}, 1 \mathrm{H}), 8.01(\mathrm{~s}, 1 \mathrm{H}), 11.58(\mathrm{~s}, 1 \mathrm{H}) ;{ }^{13} \mathrm{C}$ NMR (DMSO- $\left.d_{6}\right) \delta 21.1,40.1,61.2,70.3,84.7,87.7$, 99.5, 113.3; 127.6, 129.9, 136.8, 138.2, 142.5, 144.7, 149.5, 159.2; HRMS calcd for $\mathrm{C}_{18} \mathrm{H}_{19} \mathrm{IN}_{2} \mathrm{NaO}_{7} \mathrm{~S}[\mathrm{M}+\mathrm{Na}]^{+}$556.9850, found 556.9841.

(Z)-1-N-Benzyl-5-(1-amino-2-tosylvinyl)uracil (22). Procedure A. (E)-1- $N$-Benzyl-5-(1chloro-2-tosylvinyl)uracil $3(41.5 \mathrm{mg}, 0.1 \mathrm{mmol})$ was dissolved in $\mathrm{NH}_{3} / \mathrm{MeOH}(3 \mathrm{~mL})$ at $0{ }^{\circ} \mathrm{C}$ (ice bath). After stirring for $2 \mathrm{~h}$, volatiles were evaporated and the residue was column chromatographed (hexane/EtOAc, 6:4 $\rightarrow 1: 1)$ to give $22\left(29 \mathrm{mg}, 71 \%\right.$ ) as white solid. ${ }^{1} \mathrm{H}$ NMR $\left(\mathrm{DMSO}-d_{6}\right) \delta 2.37(\mathrm{~s}, 3 \mathrm{H}), 4.92(\mathrm{~s}, 2 \mathrm{H}), 5.26(\mathrm{~s}, 1 \mathrm{H}), 7.08(\mathrm{~s}, 2 \mathrm{H}), 7.28-7.36(\mathrm{~m}, 7 \mathrm{H}), 7.75(\mathrm{~d}, J=$ $8.2 \mathrm{~Hz}, 2 \mathrm{H}), 8.35(\mathrm{~s}, 1 \mathrm{H}) ;{ }^{1} \mathrm{H}$ NMR (DMSO- $\left.d_{6}\right) \delta 2.35(\mathrm{~s}, 3 \mathrm{H}), 4.90(\mathrm{~s}, 2 \mathrm{H}), 5.24(\mathrm{~s}, 1 \mathrm{H}), 7.28-$ $7.36(\mathrm{~m}, 7 \mathrm{H}), 7.73(\mathrm{~d}, J=8.2 \mathrm{~Hz}, 2 \mathrm{H}), 8.28(\mathrm{~s}, 1 \mathrm{H}) ;{ }^{13} \mathrm{C}$ NMR (DMSO- $\left.d_{6}\right) \delta 21.2,51.6,88.6$ 106.9, 125.7, 127.6, 128.1, 128.9, 129.8, 136.5, 142.3, 142.9, 147.1, 150.0, 150.7, 162.3; HRMS calcd for $\mathrm{C}_{20} \mathrm{H}_{19} \mathrm{~N}_{3} \mathrm{NaO}_{4} \mathrm{~S}[\mathrm{M}+\mathrm{Na}]^{+} 420.0994$, found 420.0939 . 
Analogues treatment of bromo 5 and iodo 8 with $\mathrm{NH}_{3} / \mathrm{MeOH}$ by Procedure $\mathrm{A}(1 \mathrm{~h})$ also gave Z-22 (70\%).

Note: The vinylic proton in 22 undergoes slow exchange $(4 \mathrm{~h})$ with deuterium when $\mathrm{D}_{2} \mathrm{O}$ was added into the solution of 22 in DMSO- $d_{6}$ as the intensity of the vinylic proton peak at $5.24 \mathrm{ppm}$ gradually decreased.

(E/Z)-1- $N$-benzyl-5-[1-(n-butylamino)-2-tosylvinyl]uracil $\quad$ (23). Procedure $\quad$ B. $n$ Butylamine ( $33 \mu \mathrm{L}, 0.3 \mathrm{mmol}$ ) was added to a stirred solution of $3(41.5 \mathrm{mg}, 0.1 \mathrm{mmol})$ in $\mathrm{MeOH}$ $(3 \mathrm{~mL})$ at ambient temperature. After $2 \mathrm{~h}$, the precipitated white solid was collected by vacuum filtration, washed with cold $\mathrm{MeOH}$ and dried under the vacuum to give $23(E / Z, 15: 85 ; 32 \mathrm{mg}$, 71\%). ${ }^{1} \mathrm{H}$ NMR (DMSO- $\left.d_{6}\right) \delta 0.75(\mathrm{t}, J=7.3 \mathrm{~Hz}, 0.45 \mathrm{H}), 0.85(\mathrm{~d}, J=7.3 \mathrm{~Hz}, 2.55 \mathrm{H}), 1.08-1.14$ (m, 0.3H), 1.26-1.36 (m, 2H), 1.42-1.47 (m, 1.7H), $2.33(\mathrm{~s}, 2.55 \mathrm{H}), 2.33(\mathrm{~s}, .45 \mathrm{H}), 2.89$ (dd, $J=$ 6.8,12.0 Hz, 1.7H), 2.96 (dd, $J=6.6,13.1 \mathrm{~Hz}, 0.3 \mathrm{H}), 4.87$ (s, 1.7H), 4.91 (s, 0.3H), 7.19 (d, $J=$ $8.0 \mathrm{~Hz}, 2 \mathrm{H}), 7.24-7.42(\mathrm{~m}, 6), 7.47(\mathrm{~d}, J=8.2 \mathrm{~Hz}, 2 \mathrm{H}), 7.67(\mathrm{~s}, 1 \mathrm{H}), 11.41(\mathrm{~s}, 1 \mathrm{H}) ;{ }^{13} \mathrm{C}$ NMR $\left(\mathrm{DMSO}-d_{6}\right) \delta 13.7,19.7,20.9,42.8,50.7,93.0,109.0,125.6,125.8,127.3,127.5,127.7,128.5$, 128.6, 129.1, 129.5, 136.7, 141.4, 143.2, 150.6, 161.4; HRMS calcd for $\mathrm{C}_{24} \mathrm{H}_{28} \mathrm{~N}_{3} \mathrm{O}_{4} \mathrm{~S}[\mathrm{M}+\mathrm{H}]^{+}$ 454.1795 , found 454.1793 .

(E)-1-N-Benzyl-5-(1-propylthio-2-tosylvinyl)uracil (24). Procedure C. $n$-Propylthiol (11 $\mu \mathrm{L}, 9 \mathrm{mg} 0.12 \mathrm{mmol})$ and $\mathrm{Et}_{3} \mathrm{~N}(17 \mu \mathrm{L}, 12 \mathrm{mg} 0.12 \mathrm{mmol})$ were added to a stirred solution of 3 (41.5 mg, $0.1 \mathrm{mmol})$ in $\mathrm{MeOH}(3 \mathrm{~mL})$ at ambient temperature. After $2 \mathrm{~h}$, the precipitated white solid was collected by vacuum filtration, washed with cold $\mathrm{MeOH}$ and dried under vacuum to give 24 (28 mg, 62\%) as white solid: ${ }^{1} \mathrm{H}$ NMR (DMSO- $\left.d_{6}\right) \delta 0.92(\mathrm{t}, J=7.3 \mathrm{~Hz}, 3 \mathrm{H}), 1.50-1.59$ (m, 2H), 2.38 (s, 3H), 2.80 (t, J=7.2 Hz, 3H), 4.92 (s, 2H), 6.48 (s, 1H), 7.31-7.41 (m, 7H), 7.60 $(\mathrm{d}, J=8.3 \mathrm{~Hz}, 2 \mathrm{H}), 7.86(\mathrm{~s}, 1 \mathrm{H}), 11.56(\mathrm{~s}, 1 \mathrm{H}) ;{ }^{13} \mathrm{C}$ NMR $\left(\mathrm{DMSO}-d_{6}\right) \delta 13.2,20.6,21.1,33.6$, 50.6, 108.9, 122.0, 127.1, 127.4, 127.8, 128.6, 129.5, 136.7, 138.8, 143.68, 143.73, 147.6, 150.4, 160.7; HRMS calcd for $\mathrm{C}_{23} \mathrm{H}_{25} \mathrm{~N}_{2} \mathrm{O}_{4} \mathrm{~S}_{2}[\mathrm{M}+\mathrm{H}]^{+} 457.1250$, found 457.1229 .

(Z)-5-(1-Amino-2-tosylvinyl)uridine (25). Treatment $(2 \mathrm{~h})$ of 19a $(25 \mathrm{mg}, 0.054 \mathrm{mmol})$ with $\mathrm{NH}_{3} / \mathrm{MeOH}\left(3 \mathrm{~mL}\right.$ ) by Procedure A (column chromatography; $\left.\mathrm{CHCl}_{3} / \mathrm{MeOH}, 9: 1\right)$ gave 25 (20 mg, 84\%): ${ }^{1} \mathrm{H}$ NMR (DMSO- $\left.d_{6}\right) \delta 2.37$ (s, 3H), 3.59 (ddd, $\left.J=2.8,5.3,12.3 \mathrm{~Hz}, 1 \mathrm{H}\right), 3.68$ (ddd, $J=3.0,5.0,12.3 \mathrm{~Hz}, 1 \mathrm{H}), 3.86-3.8(\mathrm{~m}, 1 \mathrm{H}), 4.00$ (q, $J=5.19 \mathrm{~Hz}, 1 \mathrm{H}), 4.10-4.14(\mathrm{~m}, 2 \mathrm{H})$, $5.11(\mathrm{~d}, J=5.6 \mathrm{~Hz}, 1 \mathrm{H}), 5.21(\mathrm{~s}, 1 \mathrm{H}), 5.29(\mathrm{t}, J=5.1 \mathrm{~Hz}, 1 \mathrm{H}), 5.44(\mathrm{~d}, J=5.4 \mathrm{~Hz}, 1 \mathrm{H}), 5.72(\mathrm{~d}, J$ $=4.2 \mathrm{~Hz}, 1 \mathrm{H}), 6.99(\mathrm{~s}, 2 \mathrm{H}), 7.35(\mathrm{~d}, J=8.1 \mathrm{~Hz}, 2 \mathrm{H}), 7.76(\mathrm{~d}, J=8.2 \mathrm{~Hz}, 2 \mathrm{H}), 8.33(\mathrm{~s}, 1 \mathrm{H}), 11.66$ $(\mathrm{s}, 1 \mathrm{H}) ;{ }^{13} \mathrm{C}$ NMR $\left(\mathrm{DMSO}-d_{6}\right) \delta 20.9,60.2,69.1,73.9,84.8,88.2,89.1,107.2,125.5,129.4$, 
141.5, 142.2, 142.3, 149.4, 150.7, 161.6; HRMS calculated for $\mathrm{C}_{18} \mathrm{H}_{22} \mathrm{~N}_{3} \mathrm{O}_{8} \mathrm{~S}[\mathrm{M}+\mathrm{H}]^{+} 440.1122$, found 440.1100

Note: The vinylic proton undergoes slow exchange $(2 \mathrm{~h})$ with deuterium when $\mathrm{D}_{2} \mathrm{O}$ was added into the solution of compound 25 in DMSO- $d_{6}$ as the intensity of the vinylic proton peak at $5.21 \mathrm{ppm}$ gradually decreased. Please see spectra of $\mathbf{2 5}$ recorded after $30 \mathrm{~min}$ ( $\sim 50 \%$ exchange) and $2 \mathrm{~h}\left(\sim 100 \%\right.$ exchange) after addition of $\mathrm{D}_{2} \mathrm{O}$ to DMSO- $d_{6}$ solution of $\mathbf{2 5}$. The deuterium exchange for the vinylic proton occurred in $1 \mathrm{~h}$ when 25 was dissolved in $\mathrm{MeOH}-d_{4}$.

Treatment of 16a $(70 \mathrm{mg}, 0.12 \mathrm{mmol})$ with $\mathrm{NH}_{3} / \mathrm{MeOH}(3 \mathrm{~mL})$ by Procedure A (14 h, column chromatography; $\left.\mathrm{CHCl}_{3} / \mathrm{MeOH}, 9: 1\right)$ also gave 25 (32 mg, 60\%) with the same spectroscopic data as described above.

$S$-[(E)-1-(Uridin-5-yl)-2-tosylvinyl]-L-cysteine ethyl ester (26). Treatment of 19a (19 mg, $0.04 \mathrm{mmol})$ with L-cysteine ethyl ester hydrogen chloride $(22.8 \mathrm{mg}, 0.12 \mathrm{mmol})$ in the presence of $\mathrm{Et}_{3} \mathrm{~N}\left(45.7 \mu \mathrm{L}, 33.2 \mathrm{mg}, 0.32 \mathrm{mmol}\right.$ ) by Procedure $\mathrm{C}$ (column chromatography; $\mathrm{CHCl}_{3} / \mathrm{MeOH}$, $95: 5 \rightarrow 9: 1)$ gave $26(E / Z, 85: 15 ; 13 \mathrm{mg}, 56 \%)$ as white solid. The $E$-isomer had: ${ }^{1} \mathrm{H}$ NMR $\left(\mathrm{DMSO}-d_{6}\right) \delta 1.20(\mathrm{t}, J=7.2 \mathrm{~Hz}, 3 \mathrm{H}), 2.40(\mathrm{~s}, 3 \mathrm{H}), 2.95(\mathrm{dd}, J=10.5,7.8 \mathrm{~Hz}, 1 \mathrm{H}), 3.09$ (dd, $J=$ $10.5,5.6 \mathrm{~Hz}, 1 \mathrm{H}), 3.53-3.58(\mathrm{~m}, 2 \mathrm{H}), 3.61-3.63(\mathrm{~m}, 1 \mathrm{H}), 3.87-3.90(\mathrm{~m}, 1 \mathrm{H}), 3.95-4.00(\mathrm{~m}, 2 \mathrm{H})$, 4.04-4.12 (m, 4H), 5.00-5.20 (m, 2H), $5.45(\mathrm{~d}, J=5.7 \mathrm{~Hz}, 1 \mathrm{H}), 5.80(\mathrm{~d}, J=5.1 \mathrm{~Hz}, 1 \mathrm{H}), 6.58(\mathrm{~s}$, $1 \mathrm{H}), 7.48(\mathrm{~d}, J=8.1 \mathrm{~Hz}, 2 \mathrm{H}), 7.68(\mathrm{~d}, J=8.3 \mathrm{~Hz}, 2 \mathrm{H}), 7.96(\mathrm{~s}, 1 \mathrm{H}), 11.50(\mathrm{~s}, 1 \mathrm{H}) ;{ }^{13} \mathrm{C} \mathrm{NMR}$ (DMSO- $\left.d_{6}\right) \delta 14.0,21.0,37.0,53.5,61.5,61.6,69.5,74.0,85.0,88.5,96.0,116.0,122.5,127.5$, $128.5,129.5,138.5,143.5,150.5,160.0,173.0$; HRMS calculated for $\mathrm{C}_{23} \mathrm{H}_{29} \mathrm{~N}_{3} \mathrm{NaO}_{10} \mathrm{~S} \mathrm{~S}_{2}[\mathrm{M}+\mathrm{Na}]^{+}$ 594.1187 , found 594.1173 .

(E)-5-(1-Propylthio-2-tosylvinyl)uridine (27). Treatment of 19a (48 mg, $0.1 \mathrm{mmol})$ with $n$ propanethiol $(11 \mu \mathrm{L}, 9 \mathrm{mg} 0.12 \mathrm{mmol})$ in the presence of $\mathrm{Et}_{3} \mathrm{~N}(17 \mu \mathrm{L}, 12 \mathrm{mg}, 0.12 \mathrm{mmol})$ by the Procedure $\mathrm{C}$ (column chromatography; $\mathrm{CHCl}_{3} / \mathrm{MeOH}, 95: 5 \rightarrow$ 9:1) gave 27 (38 mg, 75\%) as white solid: ${ }^{1} \mathrm{H}$ NMR (DMSO- $\left.d_{6}\right) \delta 0.95(\mathrm{t}, J=7.8 \mathrm{~Hz}, 3 \mathrm{H}), 1.55(\mathrm{~m}, 2 \mathrm{H}), 2.40(\mathrm{~s}, 3 \mathrm{H}), 2.80$ (t, $J$ $=7.8 \mathrm{~Hz}, 2 \mathrm{H}), 3.52-3.68(\mathrm{~m}, 2 \mathrm{H}), 3.90(\mathrm{~m}, 1 \mathrm{H}), 3.95-4.07(\mathrm{~m}, 2 \mathrm{H}), 5.04$ (brs, $1 \mathrm{H}) 5.15(\mathrm{brs}, 1 \mathrm{H})$, $5.45(\mathrm{~d}, J=5.8 \mathrm{~Hz}, 1 \mathrm{H}), 5.80(\mathrm{~d}, J=5.1 \mathrm{~Hz}, 1 \mathrm{H}), 7.38(\mathrm{~d}, J=8.1 \mathrm{~Hz}, 2 \mathrm{H}), 7.68(\mathrm{~d}, J=8.2 \mathrm{~Hz}$, 2H), $7.96(\mathrm{~s}, 1 \mathrm{H}), 8.32(\mathrm{~s}, 1 \mathrm{H}), 11.45(\mathrm{~s}, 1 \mathrm{H}) ;{ }^{13} \mathrm{C}$ NMR (DMSO- $\left.d_{6}\right) \delta$ 13.2, 20.6, 21.1, 33.6, 60.7, 69.8, 74.0, 85.0, 88.2, 109.2, 122.1, 127.1, 128.4, 129.5, 138.2, 138.8, 143.6, 150.1, 160.1; HRMS calculated for $\mathrm{C}_{21} \mathrm{H}_{26} \mathrm{~N}_{2} \mathrm{NaO}_{8} \mathrm{~S}_{2}[\mathrm{M}+\mathrm{Na}]^{+}$521.1023, found 521.1019.

Note: The vinylic proton undergoes exchange when 19a was treated with $n$-propanethiol/Et ${ }_{3} \mathrm{~N}$, in $\mathrm{MeOH}-d_{4}$ (instead of $\mathrm{MeOH}$ ) as the peak for the vinylic proton at $6.45 \mathrm{ppm}$ disappeared to give 
the deuterium labeled 27: HRMS calculated for $\mathrm{C}_{21} \mathrm{H}_{25} \mathrm{DN}_{2} \mathrm{NaO}_{8} \mathrm{~S}_{2}[\mathrm{M}+\mathrm{Na}]^{+}$522.1086, found 522.1061 .

Treatment of 19b (8 mg, $0.016 \mathrm{mmol})$ with $n$-propanethiol $(1.7 \mu \mathrm{L}, 1.5 \mathrm{mg}, 0.019 \mathrm{mmol})$ in the presence of $\operatorname{Et}_{3} \mathrm{~N}(2.6 \mu \mathrm{L}, 1.9 \mathrm{mg}, 0.019 \mathrm{mmol})$, as described above, also gave $E-27(7.2 \mathrm{mg}$, $91 \%$ ) with the identical spectroscopic data.

Treatment of 19c $(8 \mathrm{mg}, 0.015 \mathrm{mmol})$ with $n$-propanethiol $(1.6 \mu \mathrm{L}, 1.3 \mathrm{mg}, 0.017 \mathrm{mmol})$ in the presence of $\operatorname{Et}_{3} \mathrm{~N}(2.4 \mu \mathrm{L}, 1.8 \mathrm{mg}, 0.017 \mathrm{mmol})$, as described above, also gave $E-27(6.2 \mathrm{mg}$, $85 \%$ ). with the identical spectroscopic data.

(E)-5-(1-Propylthio-2-tosylvinyl)-2'-deoxyuridine (28). Treatment of 21a (30 mg, 0.07 mmol) with $n$-propanethiol $(7.4 \mu \mathrm{L}, 6.2 \mathrm{mg}, 0.08 \mathrm{mmol})$ in the presence of $\mathrm{Et}_{3} \mathrm{~N}(11.3 \mu \mathrm{L}, 8.2 \mathrm{mg}$, $0.08 \mathrm{mmol}$ ) by Procedure $\mathrm{C}$ (column chromatography; $\mathrm{CHCl}_{3} / \mathrm{MeOH}, 95: 5 \rightarrow$ 9:1) gave 28 (22 mg, 68\%): ${ }^{1} \mathrm{H}$ NMR (DMSO- $\left.d_{6}\right) \delta 0.93(\mathrm{t}, J=7.3 \mathrm{~Hz}, 3 \mathrm{H}) 1.47-1.64(\mathrm{~m}, 2 \mathrm{H}), 2.00-2.11(\mathrm{~m}, 1 \mathrm{H})$, 2.14-2.19 (m, 1H), 2.39 (s, 3H), $2.80(\mathrm{t}, J=7.2 \mathrm{~Hz}, 2 \mathrm{H}), 3.55(\mathrm{dd}, J=3.5,7.9 \mathrm{~Hz}, 2 \mathrm{H}), 3.82$ (dd, $J=3.04,6.04 \mathrm{~Hz}, 1 \mathrm{H}), 4.22-4.26(\mathrm{~m}, 1 \mathrm{H}), 5.00(\mathrm{t}, J=4.9 \mathrm{~Hz}, 1 \mathrm{H}), 5.27(\mathrm{~d}, J=4.2 \mathrm{~Hz}, 1 \mathrm{H}), 6.17$ (t, $J=6.7 \mathrm{~Hz}, 1 \mathrm{H}), 6.46(\mathrm{~s}, 1 \mathrm{H}), 7.38(\mathrm{~d}, J=8.1 \mathrm{~Hz}, 2 \mathrm{H}), 7.66(\mathrm{~d}, J=8.2 \mathrm{~Hz}, 2 \mathrm{H}), 7.89(\mathrm{~s}, 1 \mathrm{H})$, $11.51(\mathrm{~s}, 1 \mathrm{H}) ;{ }^{13} \mathrm{C}$ NMR (DMSO- $\left.d_{6}\right) \delta 13.2,20.6,21.1,33.6,39.5,61.2,70.4,84.6,87.7,109.2$, 122.3, 127.0, 127.9, 129.5, 138.8, 143.6, 148.0, 149.8, 160.1; HRMS calcd for $\mathrm{C}_{21} \mathrm{H}_{26} \mathrm{~N}_{2} \mathrm{NaO}_{7} \mathrm{~S}_{2}$ $[\mathrm{M}+\mathrm{Na}]^{+}$505.1074, found 505.1077.

$S$-[(E)-1-(2'-deoxyuridin-5-yl)-2-tosylvinyl]-L-cysteine ethyl ester (29). Treatment of 21a (30 mg, $0.07 \mathrm{mmol}$ ) with L-cysteine ethyl ester hydrogen chloride $(36 \mathrm{mg}, 0.21 \mathrm{mmol}$ ) in the presence of $\mathrm{Et}_{3} \mathrm{~N}(58 \mu \mathrm{L}, 42 \mathrm{mg} \mathrm{mg}, 0.42 \mathrm{mmol}$ ) by Procedure C (column chromatography; $\mathrm{CHCl}_{3} / \mathrm{MeOH}, 95: 5 \rightarrow$ 9:1) gave $29(E / Z, 80: 20 ; 27 \mathrm{mg}, 70 \%$, ) as a mixture of isomers. Second column chromatography gave $29(19 \mathrm{mg}, 50 \%)$ as single $E$-isomer: ${ }^{1} \mathrm{H} \mathrm{NMR}\left(\mathrm{MeOD}-d_{4}\right) \delta 1.32(\mathrm{t}$, $J=7.2 \mathrm{~Hz}, 3 \mathrm{H}), 2.14(\mathrm{ddd}, J=5.9,7.3,13.5 \mathrm{~Hz}, 1 \mathrm{H}), 2.30$ (ddd, $J=3.1,6.2,13.6 \mathrm{~Hz}, 1 \mathrm{H}), 2.43$ (s, 3H), $3.02(\mathrm{dd}, J=7.8,10.5 \mathrm{~Hz}, 1 \mathrm{H}), 3.12(\mathrm{dd}, J=5.6,10.5 \mathrm{~Hz}, 1 \mathrm{H}), 3.76(\mathrm{dd}, J=3.7,11.9 \mathrm{~Hz}$, $1 \mathrm{H}), 3.83$ (dd, $J=3.6,11.9 \mathrm{~Hz}, 1 \mathrm{H}), 3.99$ (d, $J=14.9 \mathrm{~Hz}, 1 \mathrm{H}), 4.03$ (q, $J=3.4 \mathrm{~Hz}, 1 \mathrm{H}), 4.08$ (dd, $J=5.6,7.8 \mathrm{~Hz}, 1 \mathrm{H}), 4.27$ (q, $J=7.1 \mathrm{~Hz}, 2 \mathrm{H}), 4.38(\mathrm{dt}, J=3.0,6.0 \mathrm{~Hz}, 1 \mathrm{H}), 4.75(\mathrm{~d}, J=14.9 \mathrm{~Hz}$, $1 \mathrm{H}), 6.24(\mathrm{t}, J=6.7 \mathrm{~Hz}, 1 \mathrm{H}), 7.33(\mathrm{~d}, J=8.1 \mathrm{~Hz}, 2 \mathrm{H}), 7.57(\mathrm{~d}, J=8.3 \mathrm{~Hz}, 2 \mathrm{H}), 7.99(\mathrm{~s}, 1 \mathrm{H}) ;{ }^{13} \mathrm{C}$ NMR (MeOH- $\left.d_{4}\right) \delta 14.5,21.7,37.1,41.7,62.8,63.3,63.6,67.1,72.7,74.5,87.0,89.4,116.4$, 130.0, 130.9, 137.1, 138.1, 146.5, 151.6, 163.6, 172.8; HRMS calcd for $\mathrm{C}_{23} \mathrm{H}_{29} \mathrm{~N}_{3} \mathrm{NaO}_{9} \mathrm{~S}_{2}$ $[\mathrm{M}+\mathrm{Na}]^{+}$for 578.1237 , found 578.1239 . 


\section{Kinetic Analysis.}

Kinetic data for the reactions between $\beta$-halovinylsulfone 19a-19c and $n$-propanethiol in the presence of TEA were acquired by ${ }^{1} \mathrm{H}$ NMR at room temperature using a Bruker $400 \mathrm{MHz}$ spectrometer. For each experiment propanethiol $\left(200 \mu \mathrm{L}, 29.8 \mathrm{mM}\right.$ solution in DMSO- $\left.d_{6}\right)$ was added to NMR tube containing $200 \mu \mathrm{L}$ of the $29.8 \mathrm{mM}$ solution of $\beta$-halovinylsulfone (19a-19c) in $\mathrm{DMSO}-d_{6}$ and the proper ratio of PrSH to substrates 19a-c was confirmed by proton integration. Then TEA $\left(200 \mu \mathrm{L}, 29.8 \mathrm{mM}\right.$ solution in DMSO- $\left.d_{6}\right)$ was added for the total $9.93 \mathrm{mM}$ concentration of substrates and the reaction mixture was scanned once every 5 minutes over first 30 minutes and later every $10 \mathrm{~min}$. This procedure was repeated in duplicate. For each of the halovinylsulfones 19a-c tested, proton spectra showed formation of the substitution product $E-27$. The profile for the reaction was measured by integrating disappearance of the signal of $\mathrm{H} 6$ of substrate 19a-c at 8.25, 8.21, and $8.07 \mathrm{ppm}$ and appearance of $\mathrm{H} 6$ signal at $7.96 \mathrm{ppm}$ for the product $E-27$ on ${ }^{1} \mathrm{H}$ NMR spectra. The y-intercept for each plot was set up to $1 /[$ starting $\beta$ halovinylsulfone concentration] (Figure S1). Plots show data collected within $\sim 40 \%$ (19a), 55\% (19b) and $\sim 60 \%$ (19c) conversion to the $\beta$-(propylthio)vinylsulfone $(E-27)$ product.

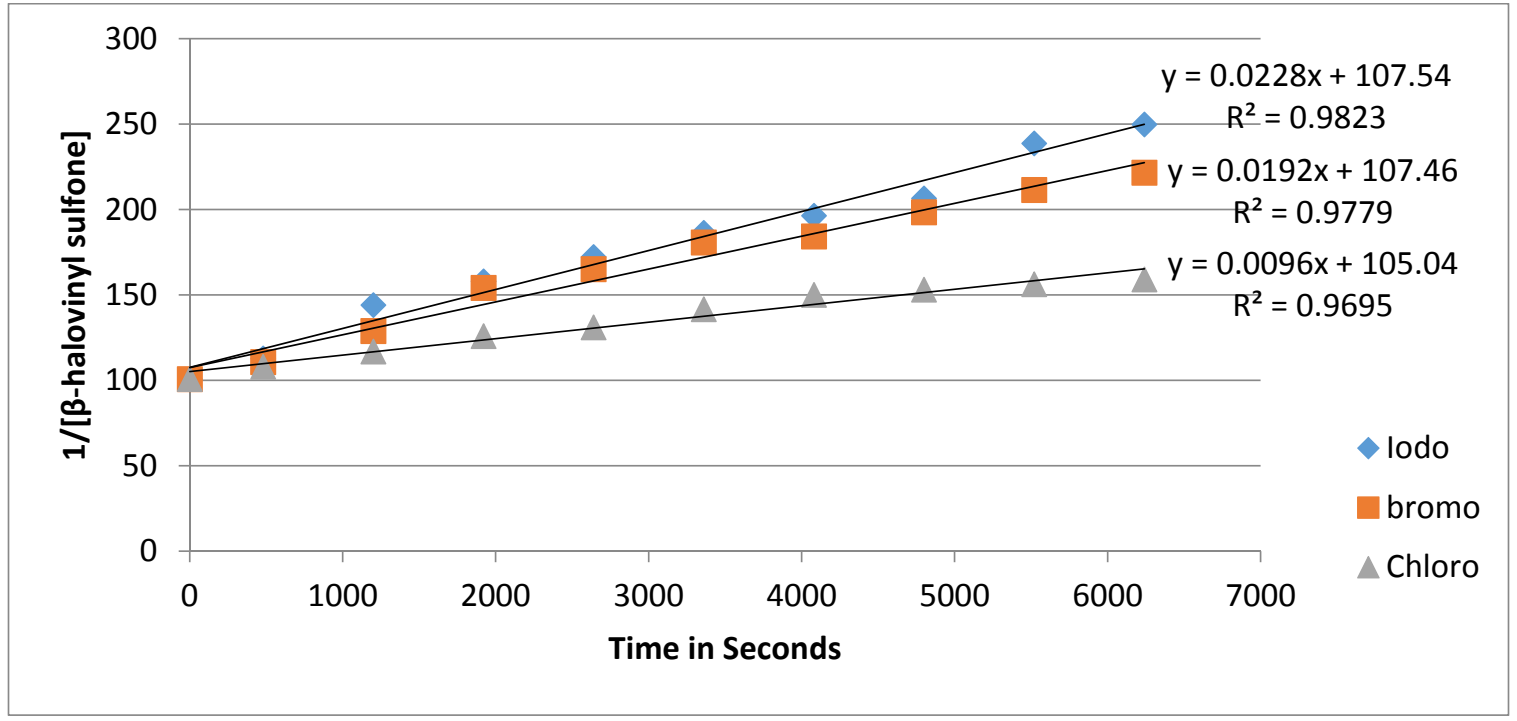

Figure S1. The rate plots for the reactions between $\beta$-halovinylsulfone 19a (chloro), 19b (bromo), and 19c (iodo) with $n$-propanethiol in the presence of TEA. The second order rate constant was calculated by plotting $1 /[\beta$-halovinylsulfone $]$ as a function of time. 


\section{Single-crystal X-ray crystallography}

Colorless cuboidal crystals of 19a and colorless plate-like crystals of 24 were obtained by the slow evaporation of the respective compounds from the acetonitrile solutions. Single crystal X-ray data were collected on a Bruker D8 Quest instrument equipped with a CMOS detector. The structures were solved by intrinsic phasing methods available with SHELXT, and were refined by full-matrix least-squares using SHELXS, and SHELXL program packages supplied within the APEX3 suite. $^{\mathrm{S} 9}$ Multi-scan absorption correction was performed using SADABS. ${ }^{\mathrm{S} 10}$ Crystallographic data have been submitted to the Cambridge Crystallographic Data Center (CCDC numbers: 1419905 (19a) and 1447952 (24) and can be obtained free of charge from www.ccdc.cam.ac.uk/data_request/cif. Crystallographic data are provided in Table S1 and the Xray crystal structure for 19a and $\mathbf{2 4}$ are shown in Figure S2 and Figure S3, respectively.

Compound 19a crystallizes with four lattice water molecules that are in various strengths of H-bonding with hydroxyl groups and/or oxygen atoms from ribose ring, uracil ring as well as sulfonyl group. Crystal structure of $\mathbf{2 4}$ had S-propyl and phenyl rings disordered around C-C single bonds and were refined using the PARTS routine in SHELXL.

Table S1. Crystallographic data and structure refinement.

\begin{tabular}{lll}
\hline & $\boldsymbol{E - 1 9 a}$ & $\boldsymbol{E}-24$ \\
\hline Formula & $\mathrm{C}_{18} \mathrm{H}_{27} \mathrm{ClN}_{2} \mathrm{O}_{12} \mathrm{~S}$ & $\mathrm{C}_{23} \mathrm{H}_{24} \mathrm{~N}_{2} \mathrm{O}_{4} \mathrm{~S}_{2}$ \\
$\boldsymbol{f}_{\mathbf{w}}(\mathbf{a m u})$ & 530.92 & 456.56 \\
Temperature (K) & $199(2)$ & $292(2)$ \\
Size $\left(\mathbf{m m}^{3}\right)$ & $0.23 \times 0.20 \times 0.19$ & $0.26 \times 0.12 \times 0.03$ \\
Crystal System & Monoclinic & Monoclinic \\
Space group & $P 2_{1}$ & $P 2{ }_{1} / \mathrm{n}$ \\
$\boldsymbol{a}(\AA)$ & $7.2371(7)$ & $8.2793(12)$ \\
$\boldsymbol{b}(\AA)$ & $9.8802(10)$ & $22.107(4)$ \\
$\boldsymbol{c}(\AA)$ & $15.9674(14)$ & $12.8092(19)$ \\
$\boldsymbol{\alpha}\left({ }^{\circ}\right)$ & 90 & 90 \\
$\boldsymbol{\beta}\left({ }^{\circ}\right)$ & $97.002(2)$ & $105.304(3)$ \\
$\boldsymbol{\gamma}\left({ }^{\circ}\right)$ & 90 & 90
\end{tabular}




\begin{tabular}{|c|c|c|}
\hline$V\left(\AA^{3}\right)$ & $1133.2(2)$ & $2261.4(6)$ \\
\hline$Z$ & 2 & 4 \\
\hline$\rho_{\text {calc }}\left(\mathrm{g} \mathrm{cm}^{-3}\right)$ & 1.556 & 1.341 \\
\hline$\mu\left(\mathbf{m m}^{-1}\right)$ & 0.329 & 0.268 \\
\hline Total data & 10650 & 28387 \\
\hline Unique data & 4530 & 4742 \\
\hline Theta range $\left({ }^{\circ}\right)$ & $2.937-26.350$ & $3.147-26.659$ \\
\hline Completeness (\%) & 99.7 & 99.5 \\
\hline $\begin{array}{l}\text { Goodness of fit } \\
\text { (GOF) on } F^{2}\end{array}$ & 1.156 & 1.027 \\
\hline$R_{1} / w R_{2}(I>2 \sigma(I))$ & $0.0597 / 0.1166$ & $0.759 / 0.1066$ \\
\hline$R_{1} / w R_{2}$ (all data) & $0.0764 / 0.1251$ & $0.1759 / 0.1320$ \\
\hline $\begin{array}{l}\text { Max. and min. } \\
\text { trans. }\end{array}$ & 0.94 and 0.76 & $\begin{array}{l}0.6043 \text { and } \\
0.7454\end{array}$ \\
\hline $\begin{array}{l}\text { Largest diff. peak } \\
\text { and hole }\left(\mathrm{e} . \AA^{-3}\right)\end{array}$ & 0.438 and -0.319 & 0.248 and -0.286 \\
\hline
\end{tabular}




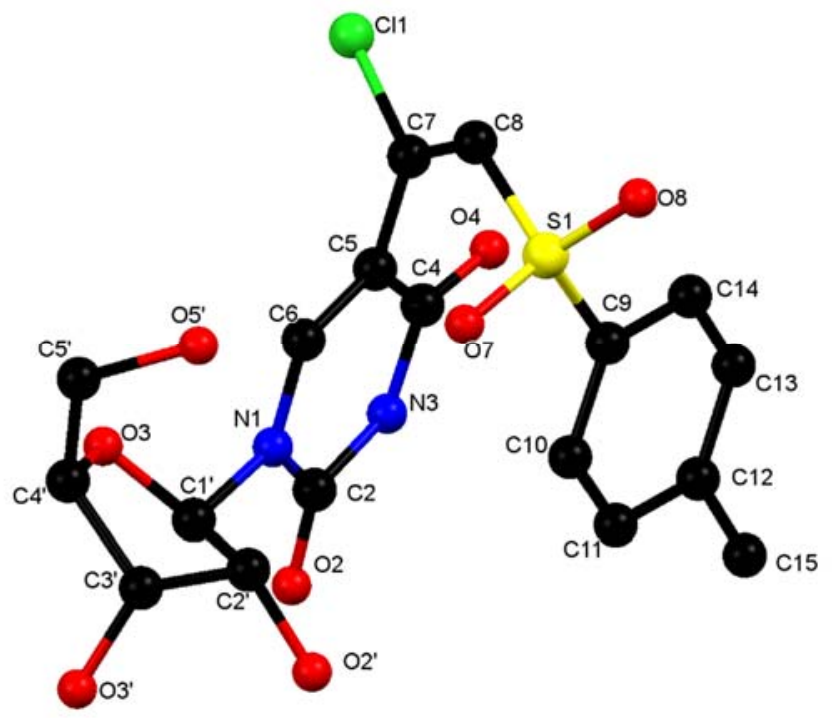

Figure S2. Ball-and-stick representation of 19a showing the atom labeling scheme. H-atoms and interstitial $\mathrm{H}_{2} \mathrm{O}$ molecules are omitted for clarity.

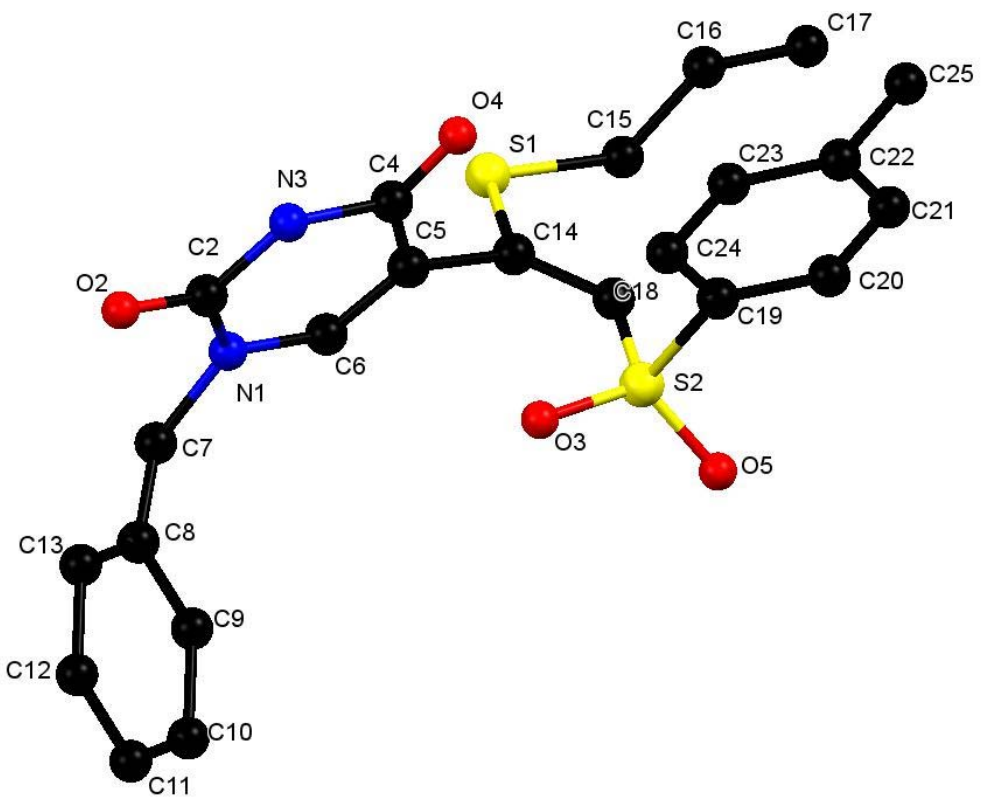

Figure S3. Ball-and-stick representation of $\mathbf{2 4}$ showing the atom labeling scheme. Disordered parts and $\mathrm{H}$-atoms are not shown for clarity. 


\section{References}

(S1) Robins, M. J.; Barr, P. J. J. Org. Chem. 1983, 48, 1854.

(S2) Robins, M. J.; Manfredini, S.; Wood, S. G.; Wanklin, R. J.; Rennie, B. A.; Sacks, S. L. J. Med. Chem. 1991, 34, 2275.

(S3) Liang, Y.; Pitteloud, J.-P.; Wnuk, S. F. J. Org. Chem. 2013, 78, 5761.

(S4) Prachayasittikul, S.; Sornsongkhram, N.; Pingaew,PR.; Worachartcheewan, A.; Ruchirawat, S.; Prachayasittikul, V. Molecules 2009, 14, 2768.

(S5) Park, S. M.; Shen, Y.; Kim, B. H. Org. Biomol. Chem. 2007, 5, 610.

(S6) Sharma, R. A.; Kavai, I.; Hughes, R. G.; Bobek, M. J. Med. Chem. 1984, 27, 410.

(S7) Barr, P. J.; Jones, A. S.; Serafinowski, P.; Walker, R. T. J. Chem. Soc., Perkin Trans. 1 1978, 1263.

(S8) Cristofoli, W. A.; Wiebe, L. I.; De Clercq, E.; Andrei, G.; Snoeck, R.; Balzarini, J.; Knaus, E. E. J. Med. Chem. 2007, 50, 2851.

(S9) Sheldrick, G. M. Acta Crystallogr. Sect. C 2015, C71, 3.

(S10) Krause, L.; Herbst-Irmer, R.; Sheldrick, G. M.; Stalke, D. J. Appl. Crystal. 2015, 48, 3. 
Page |S16

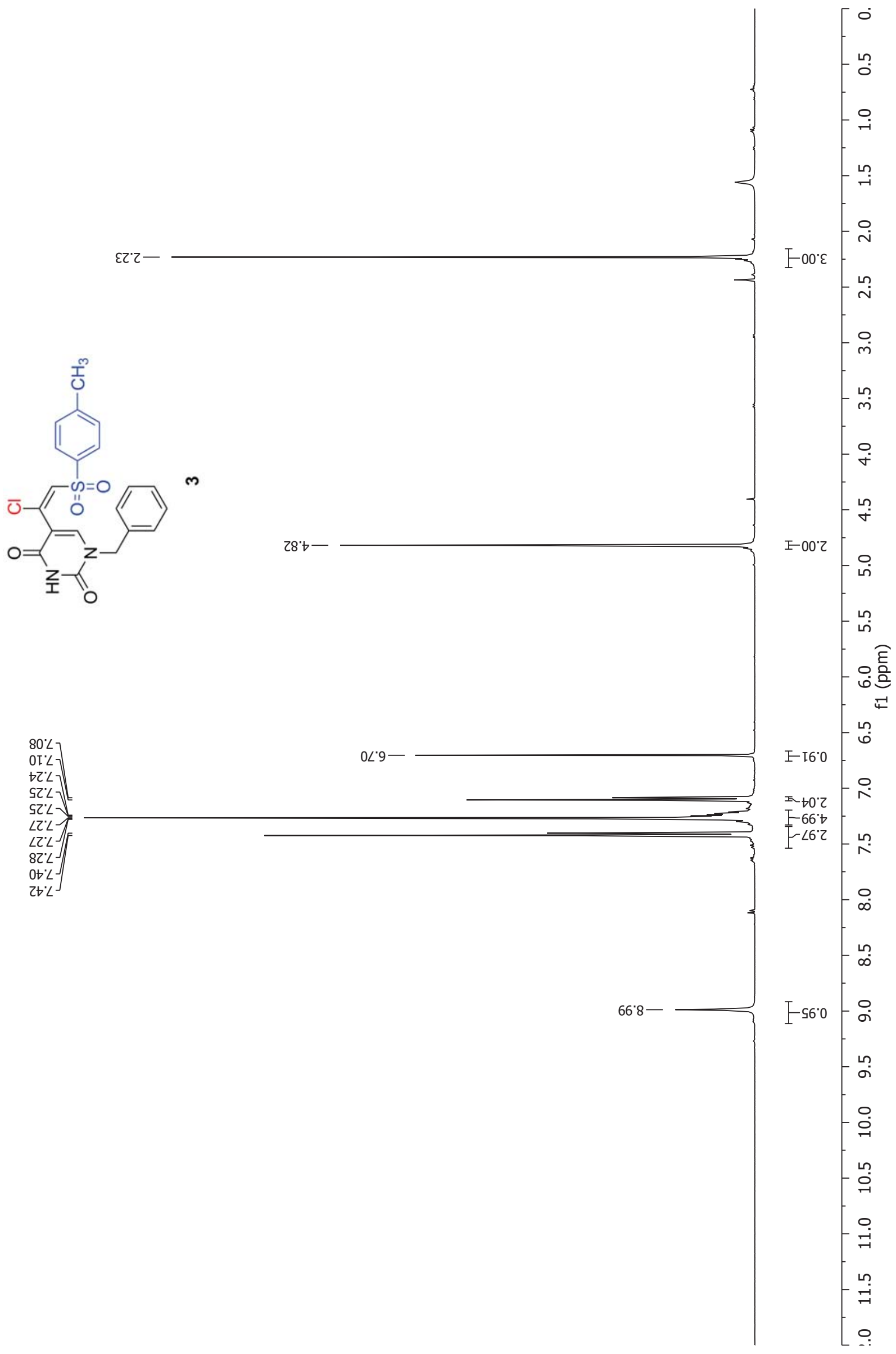


Page |S17
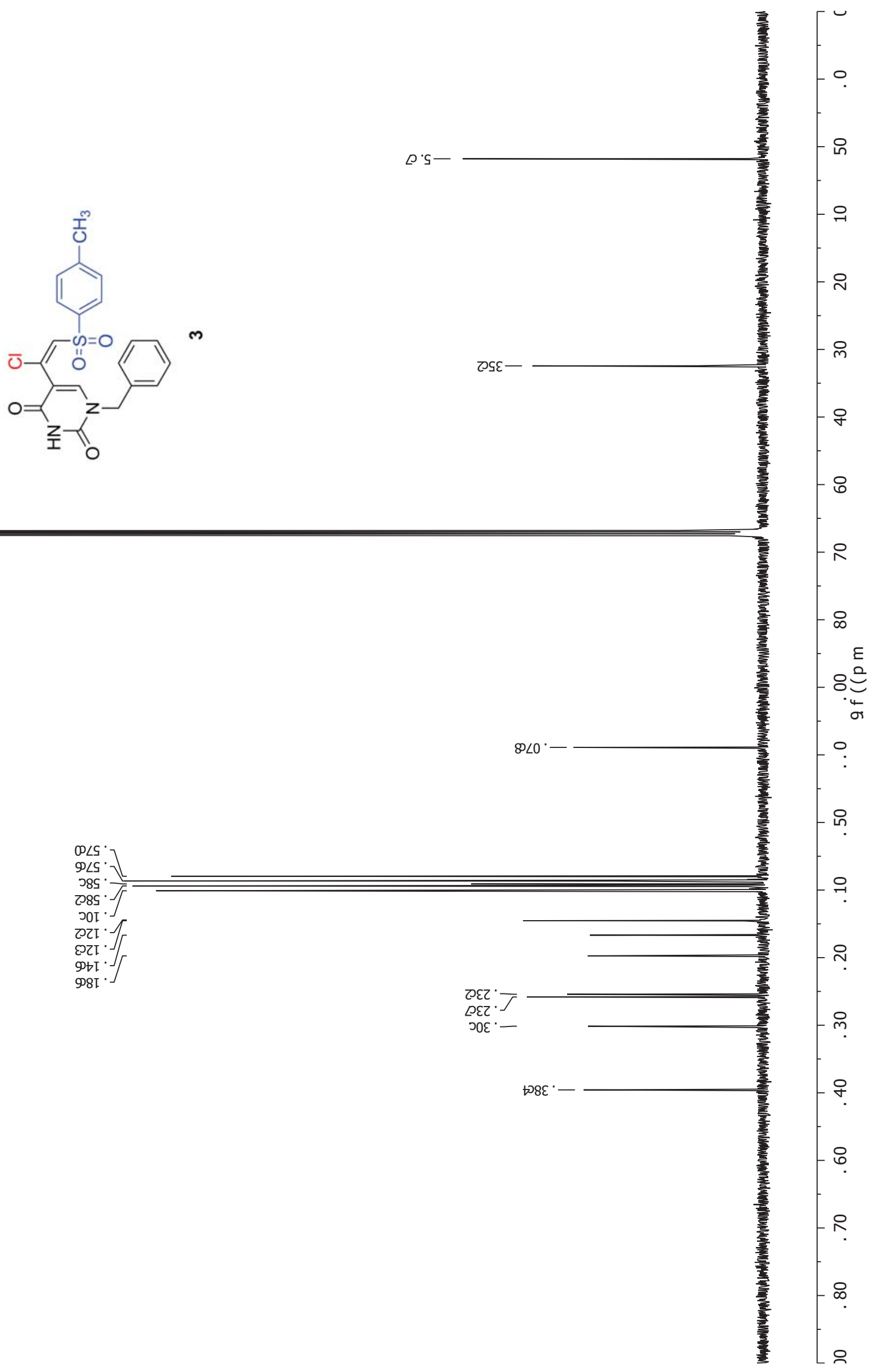
Page |S18

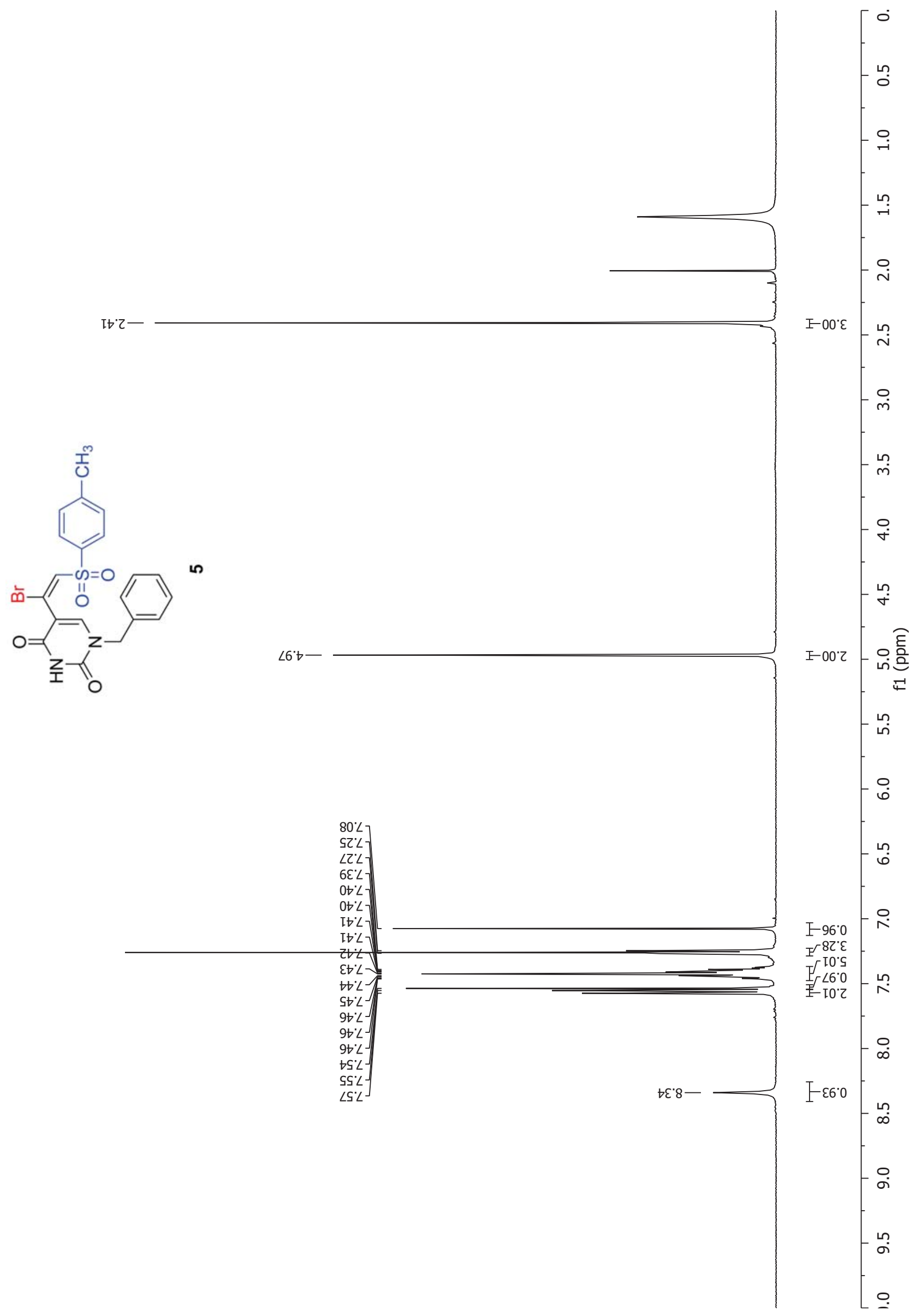


Page |S19
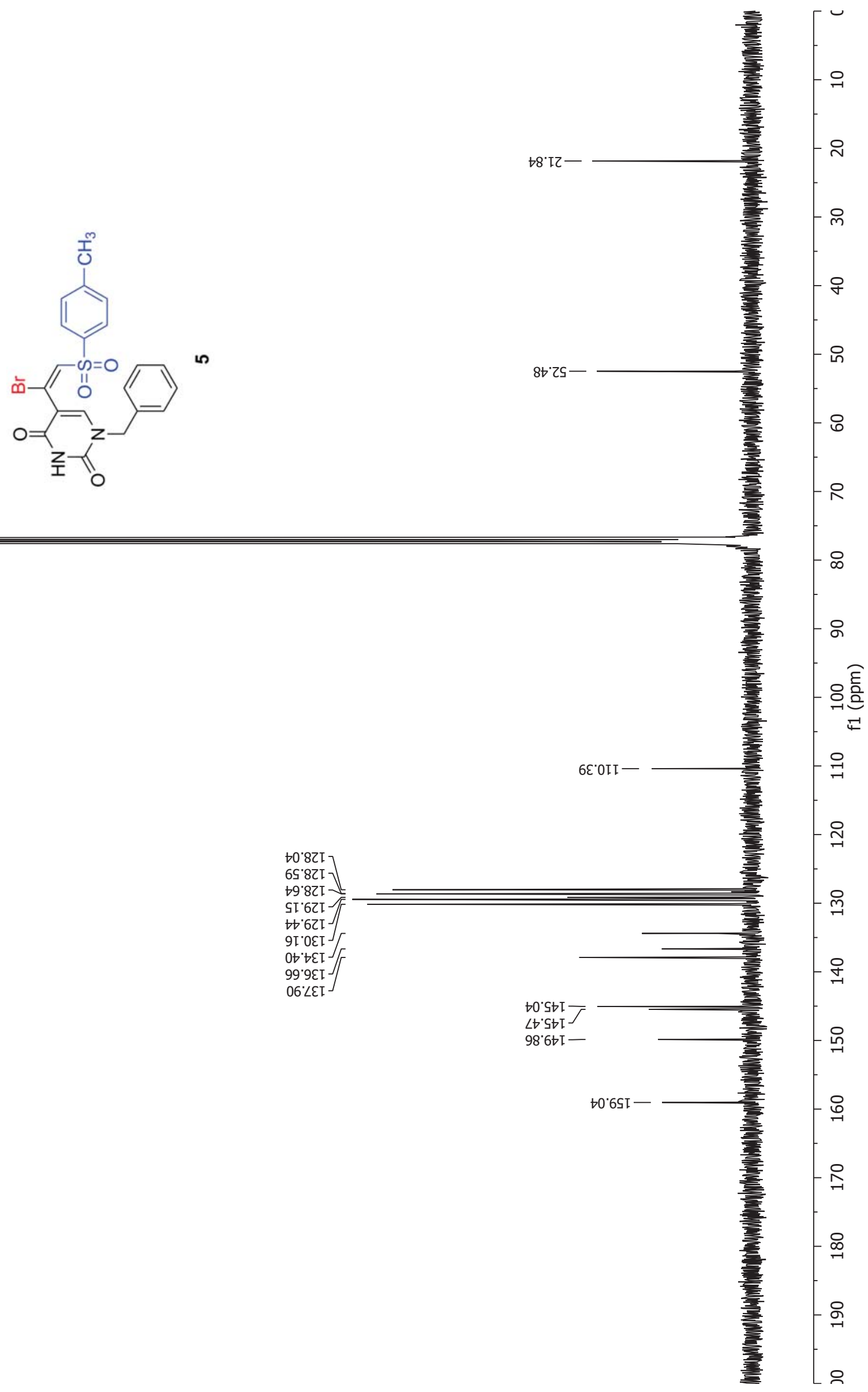
Page |S20

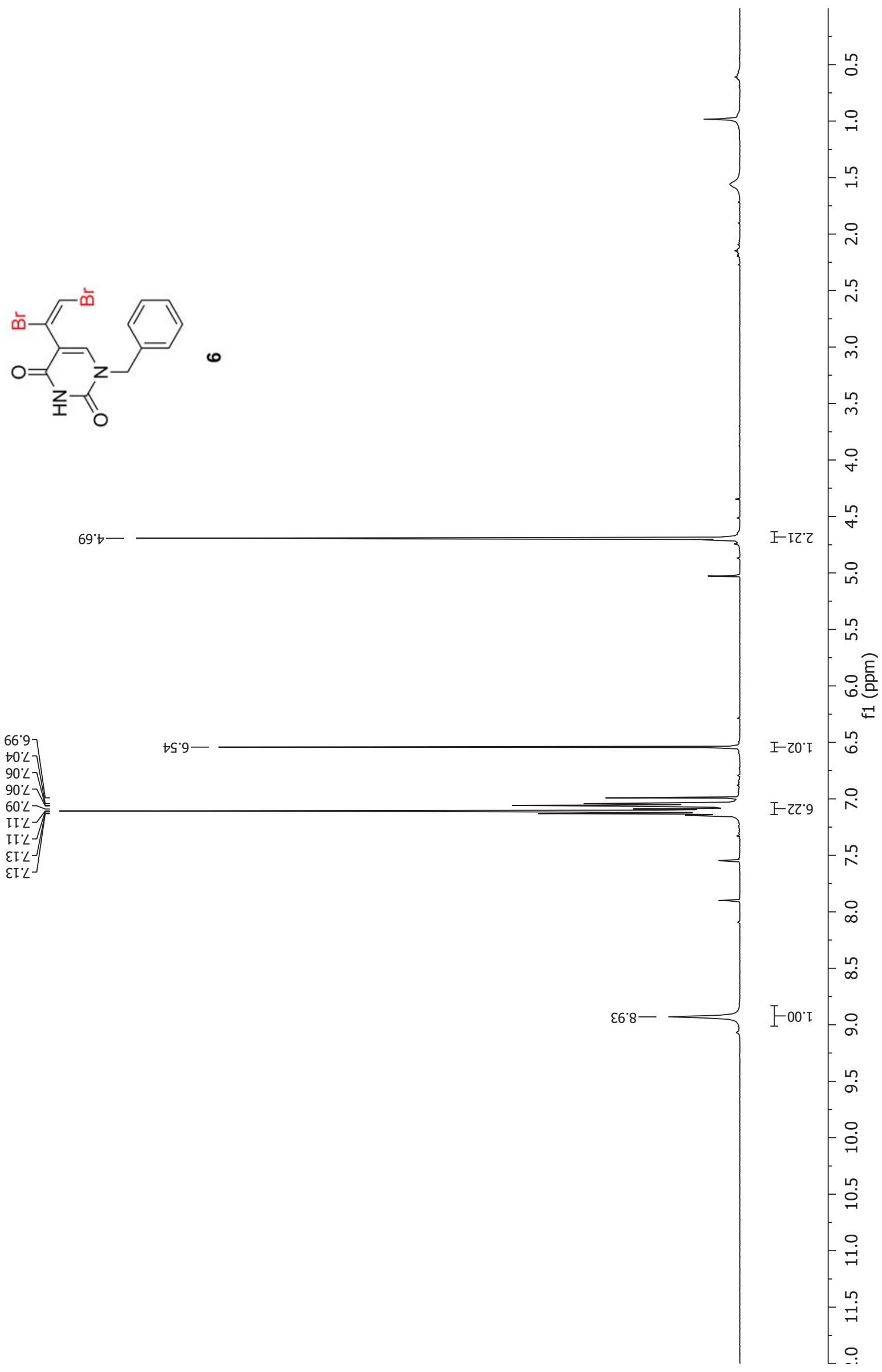


Page |S21

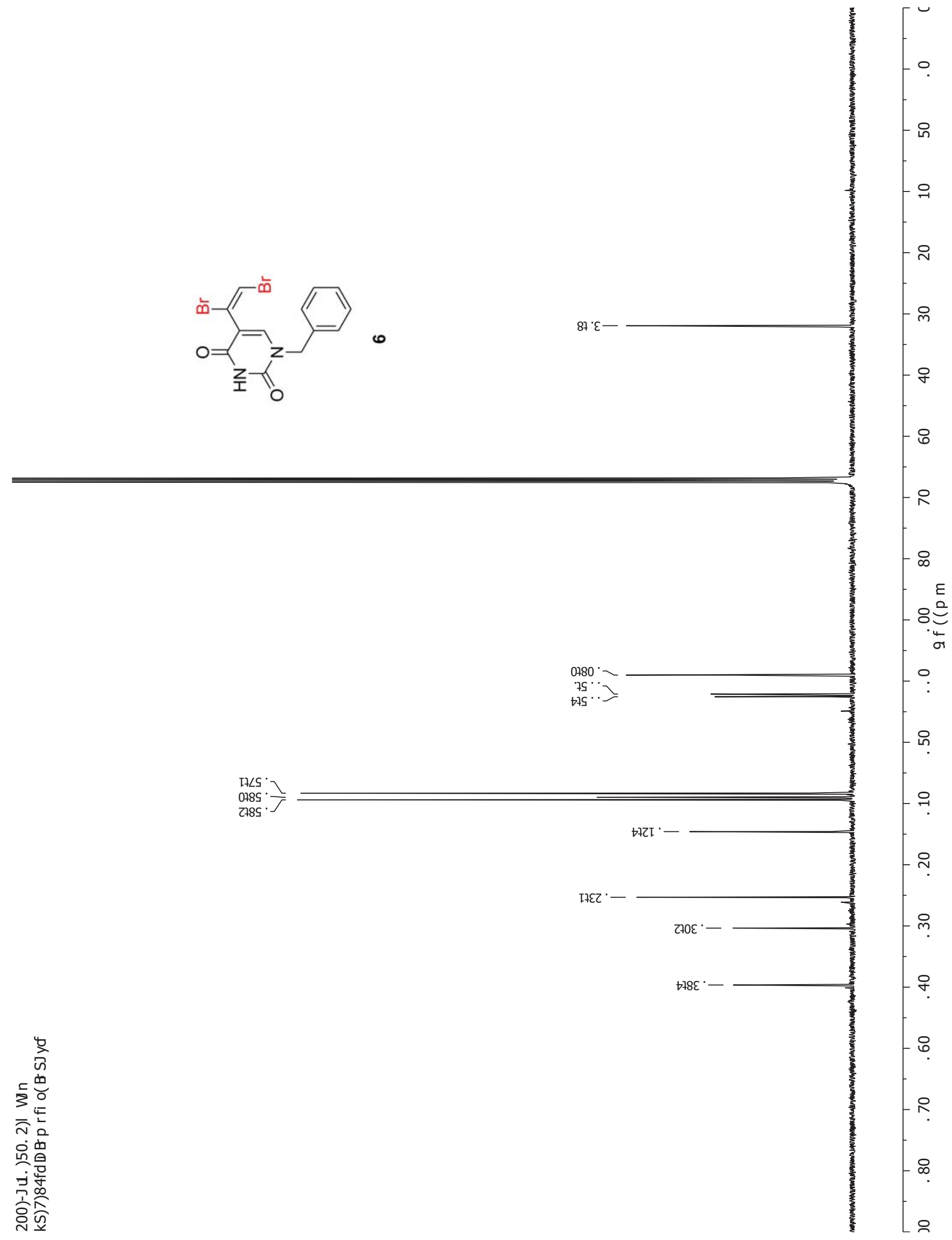


Page |S22

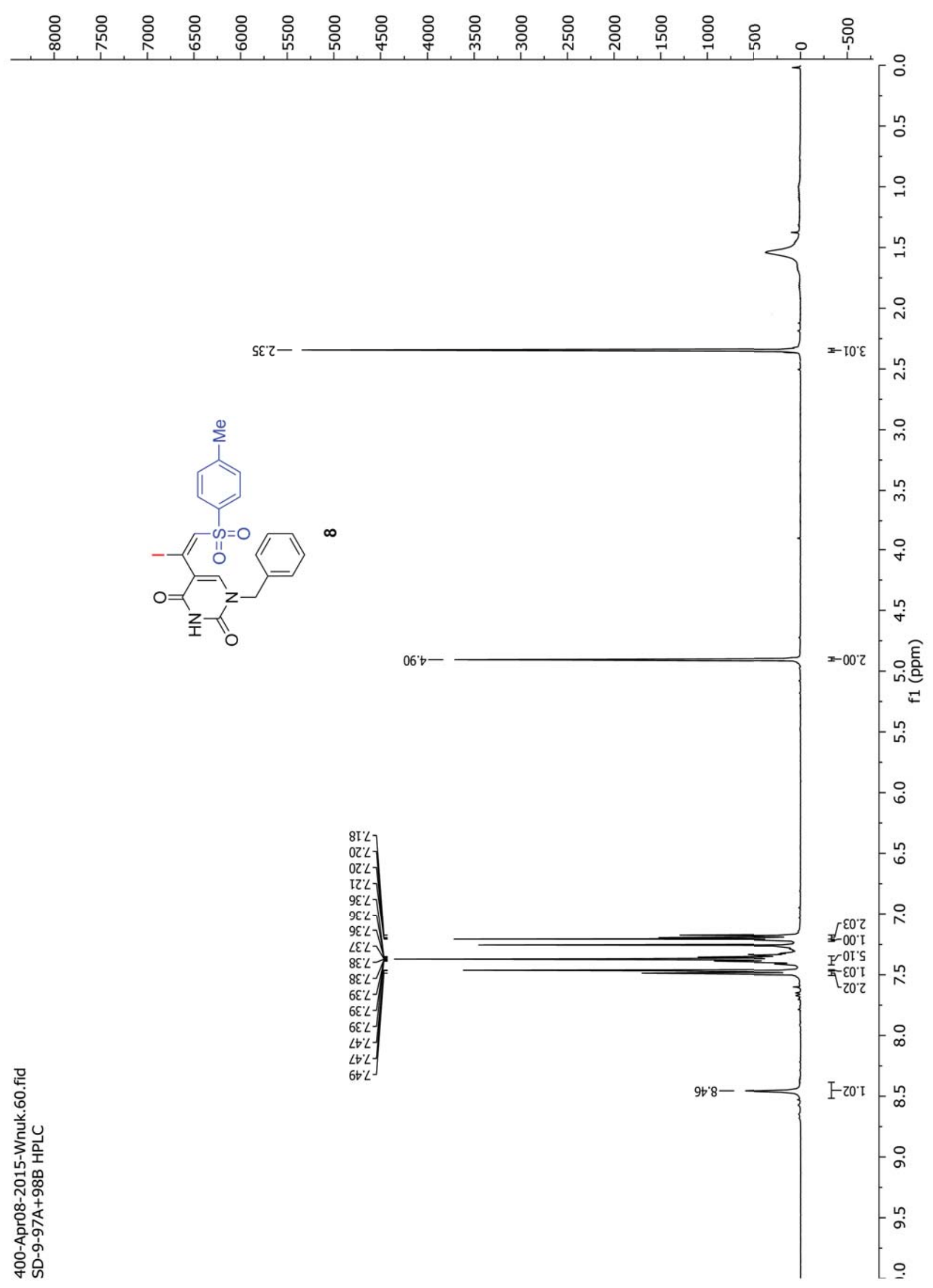


Page |S23

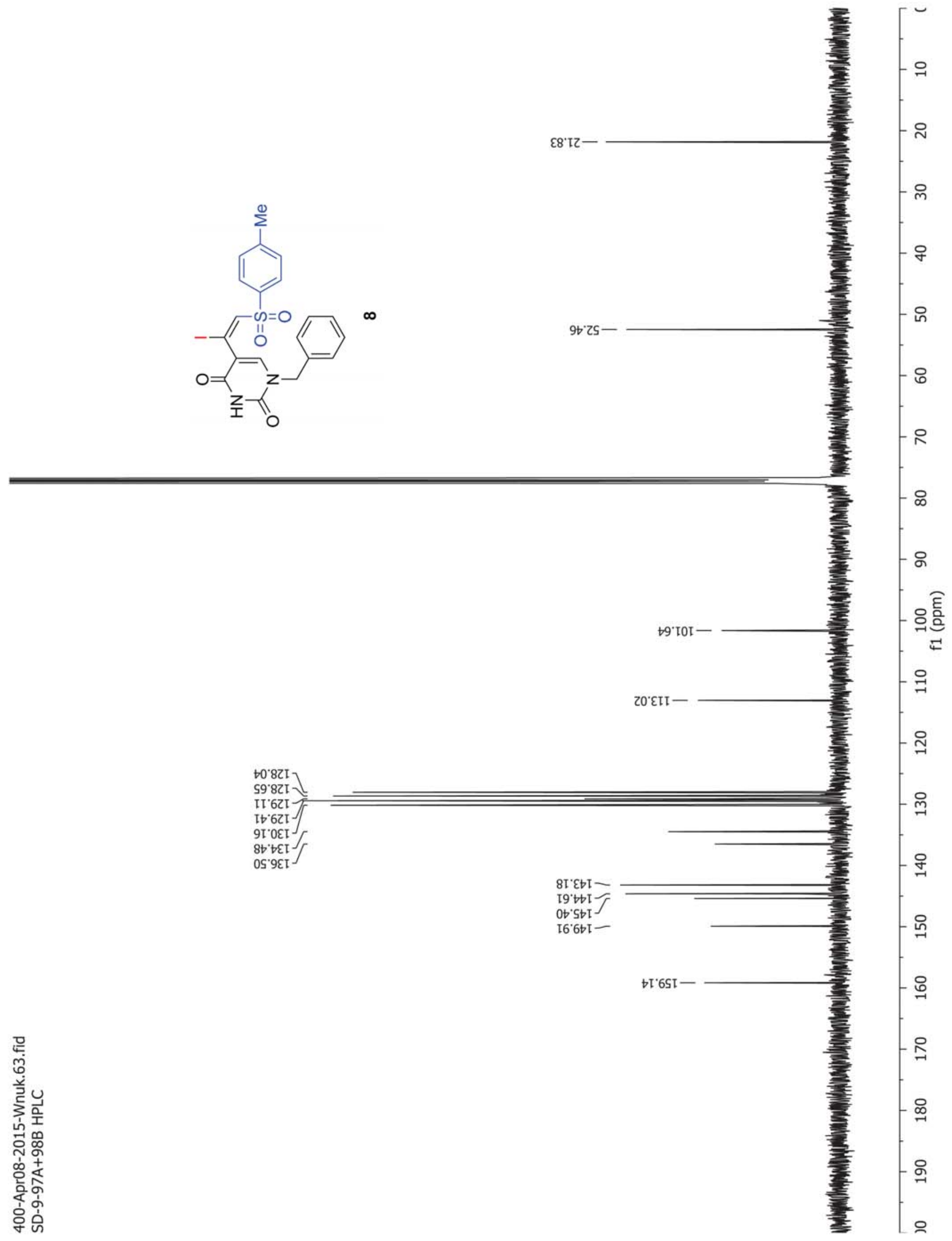


Page |S24

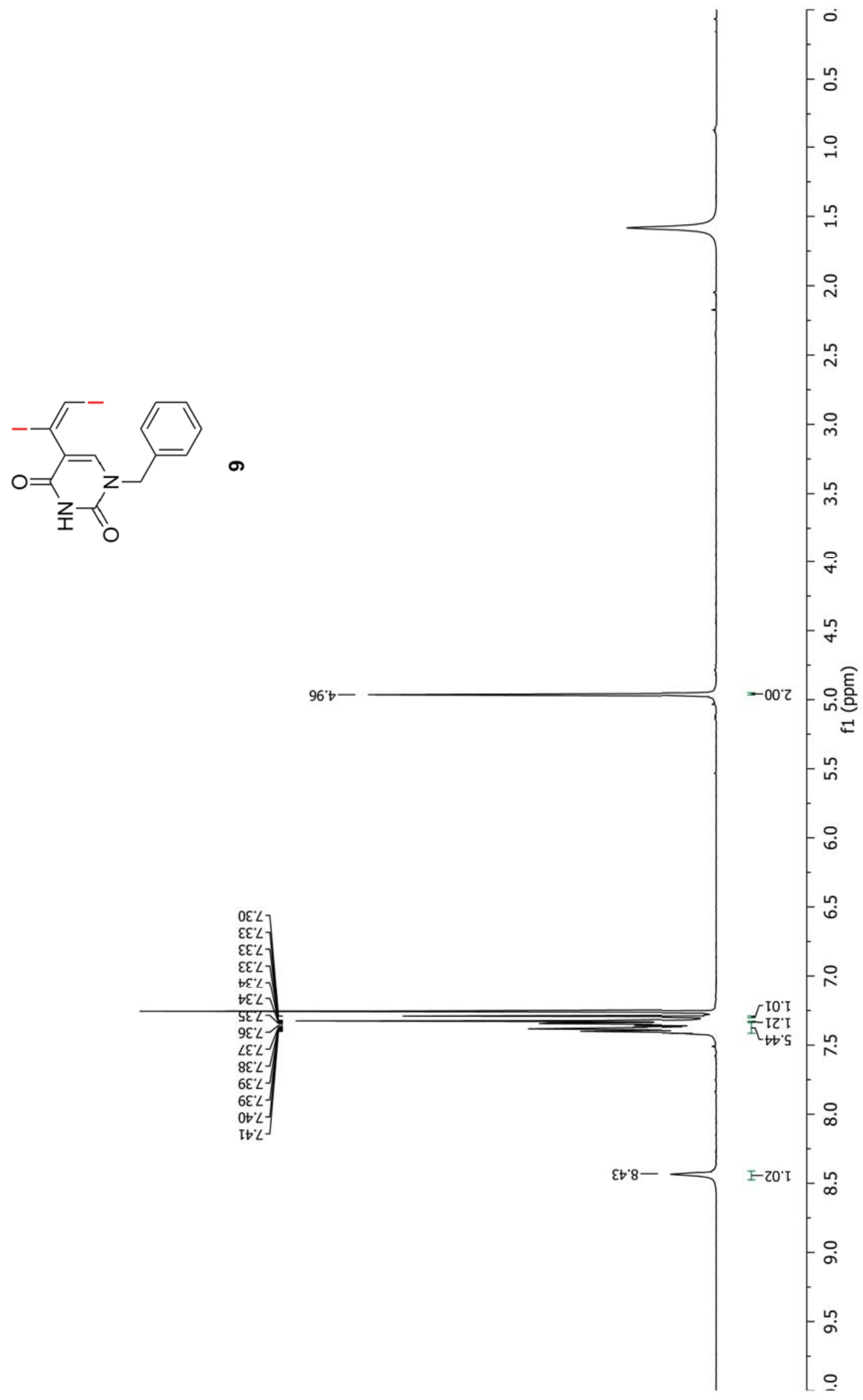


Page |S25

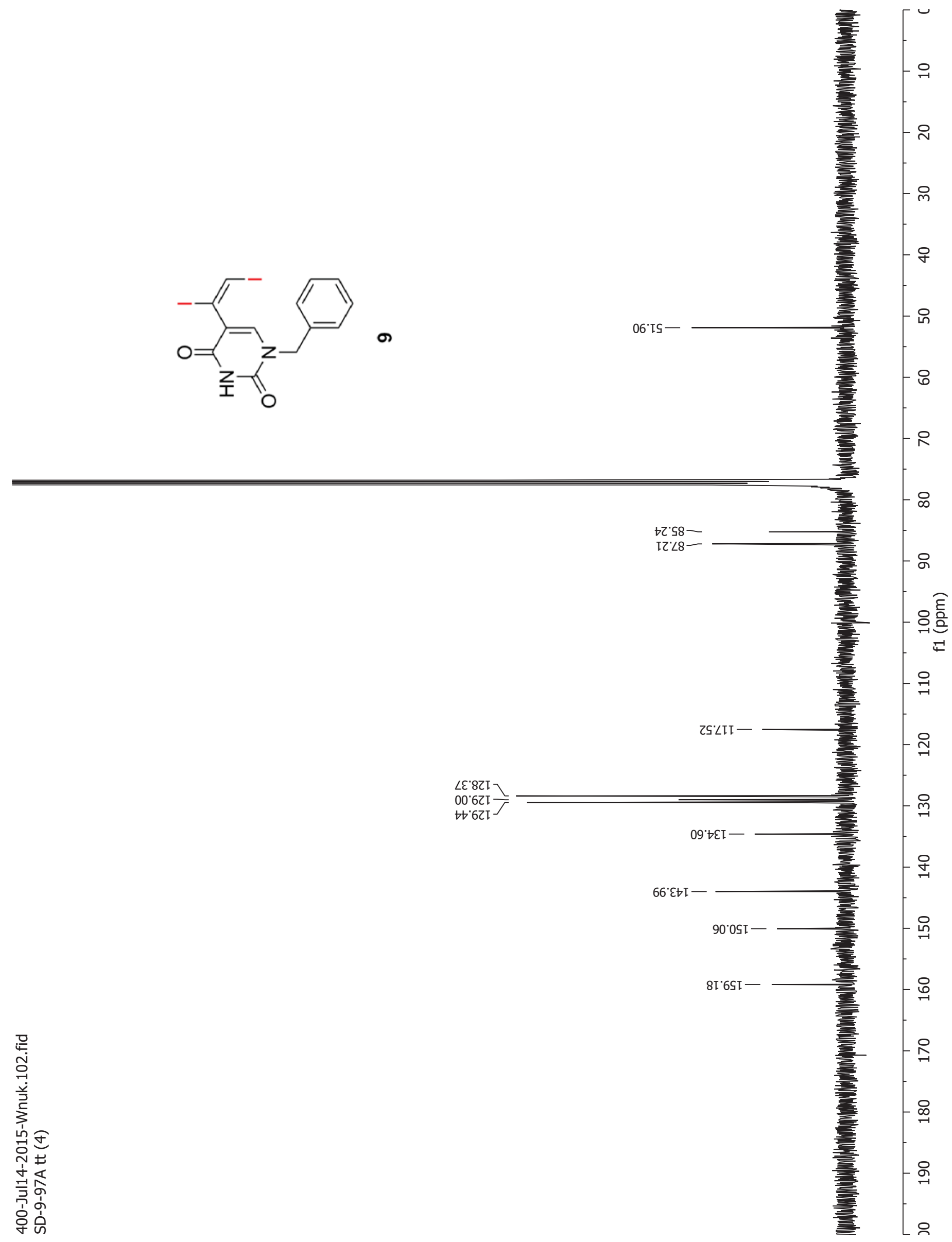


Page |S26

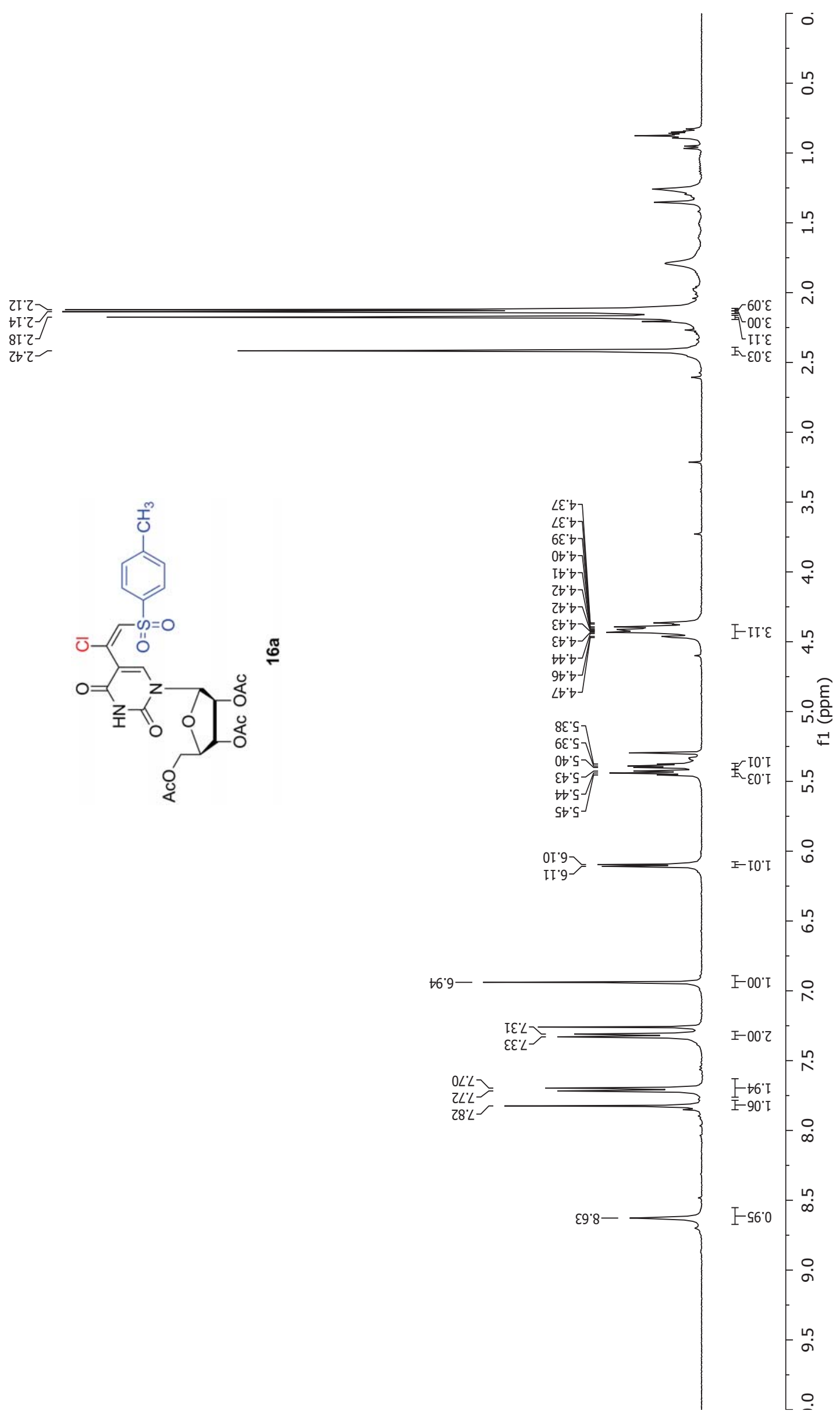


Page |S27

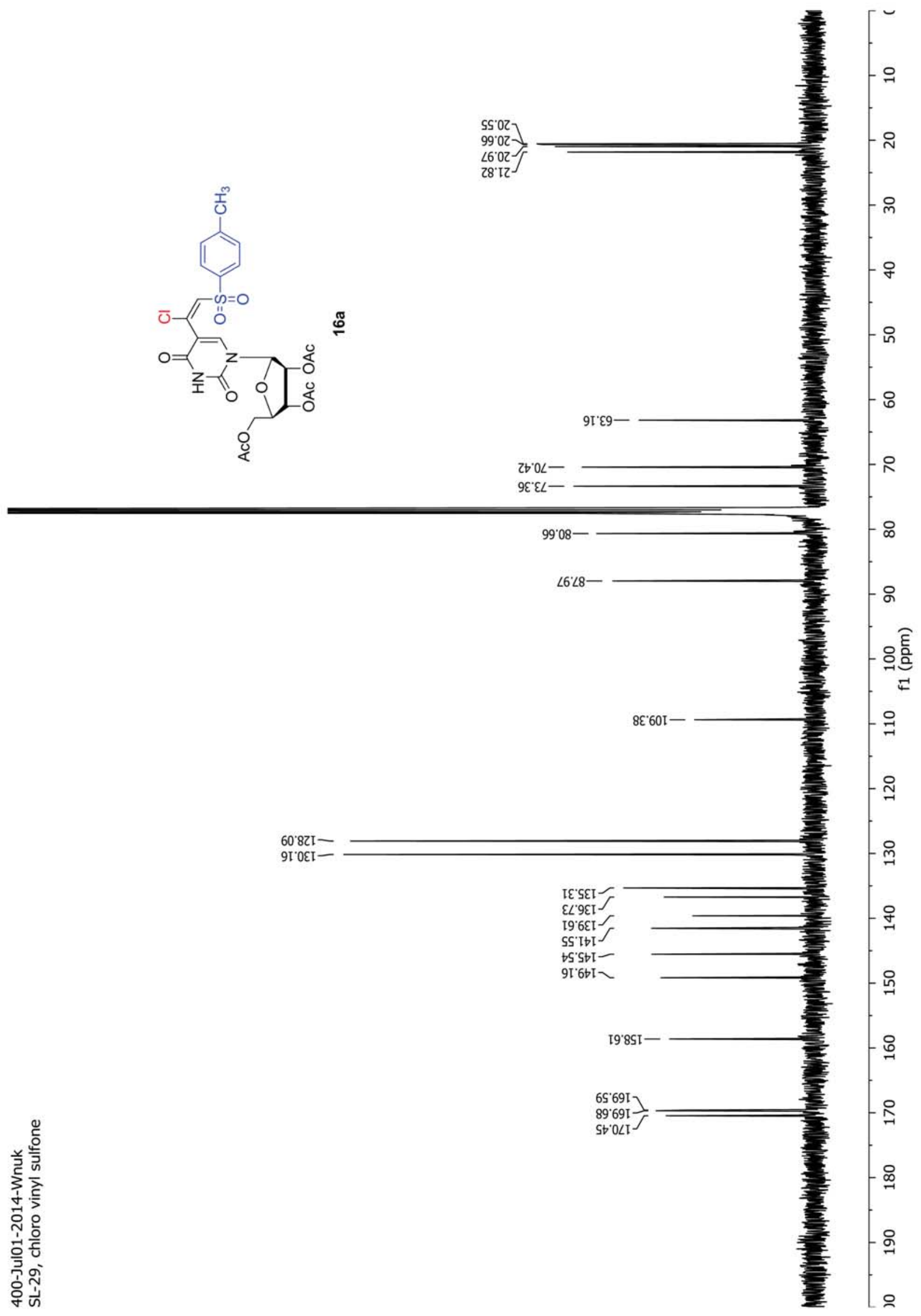


Page |S28

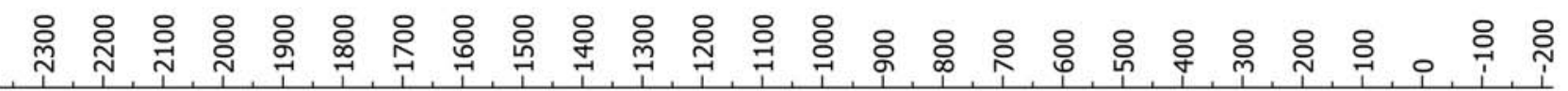

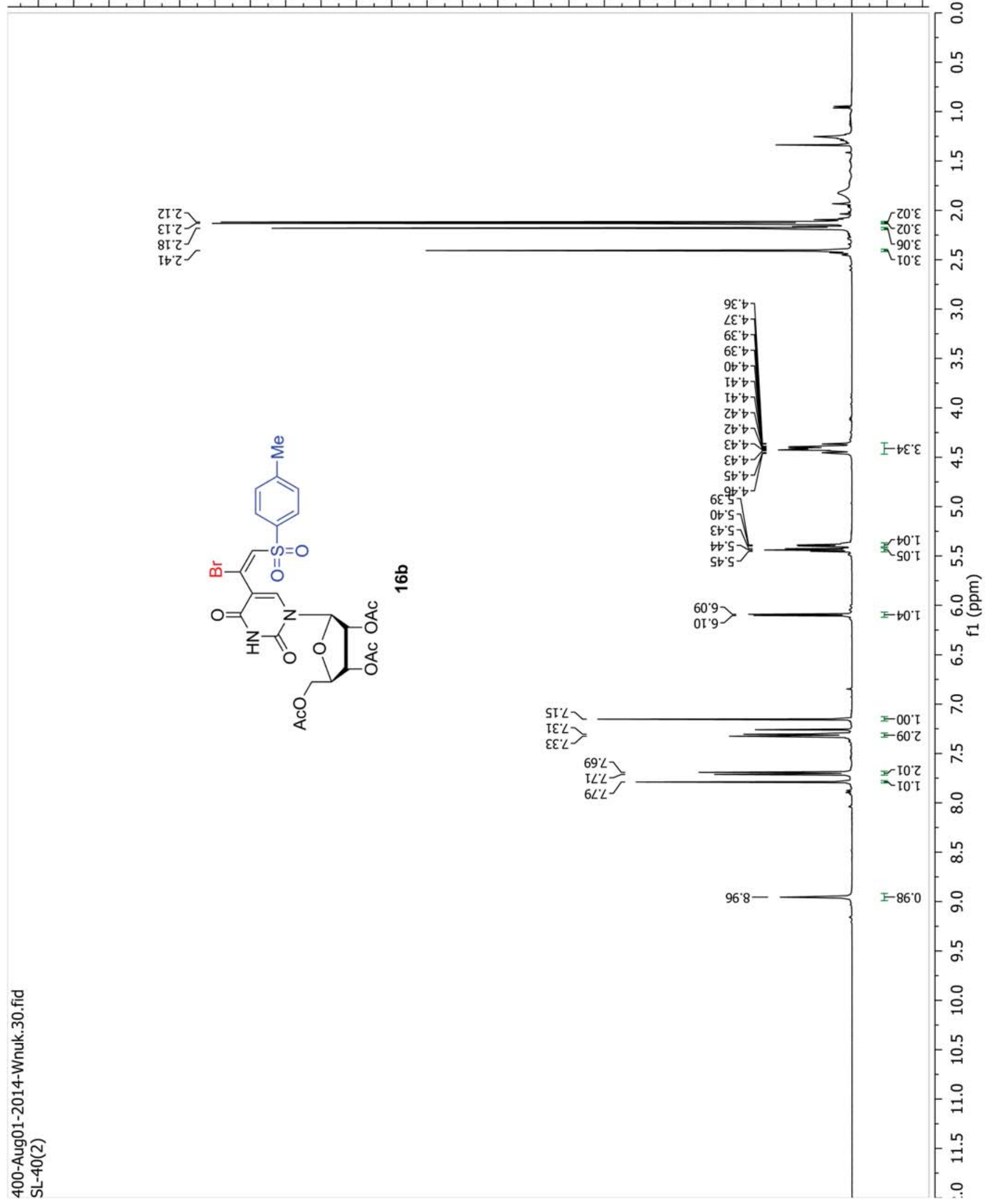


Page |S29

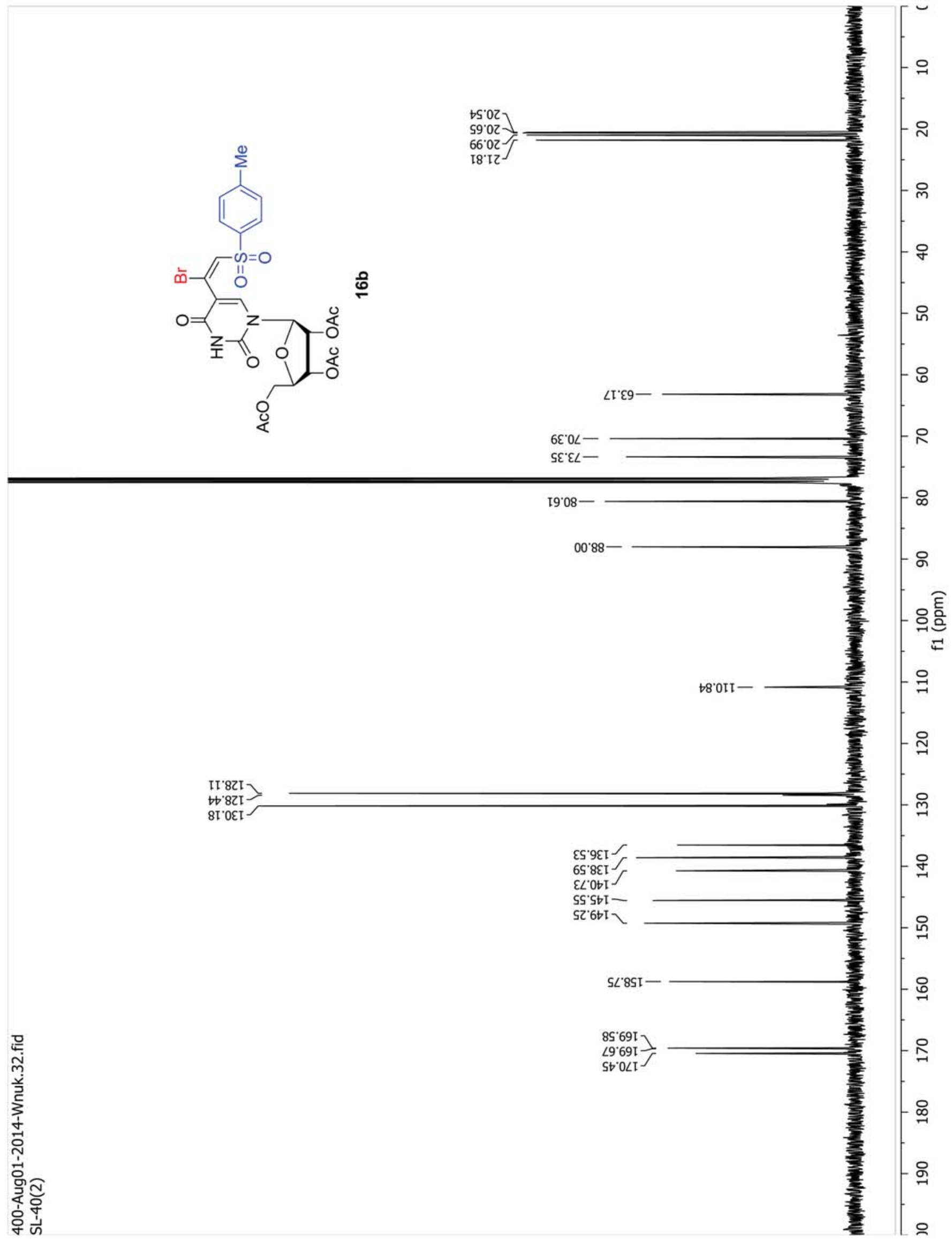


Page |S30

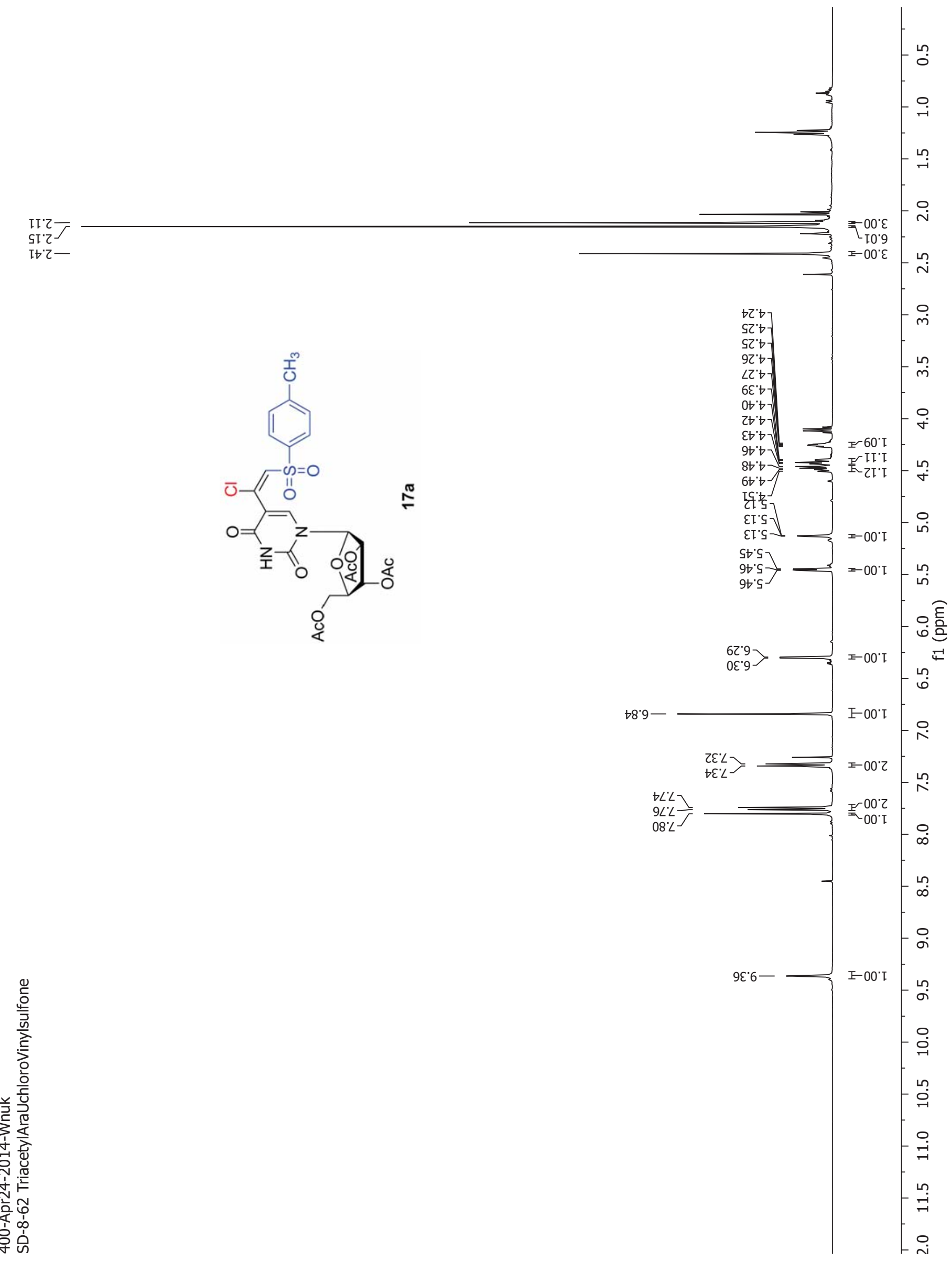


Page |S31

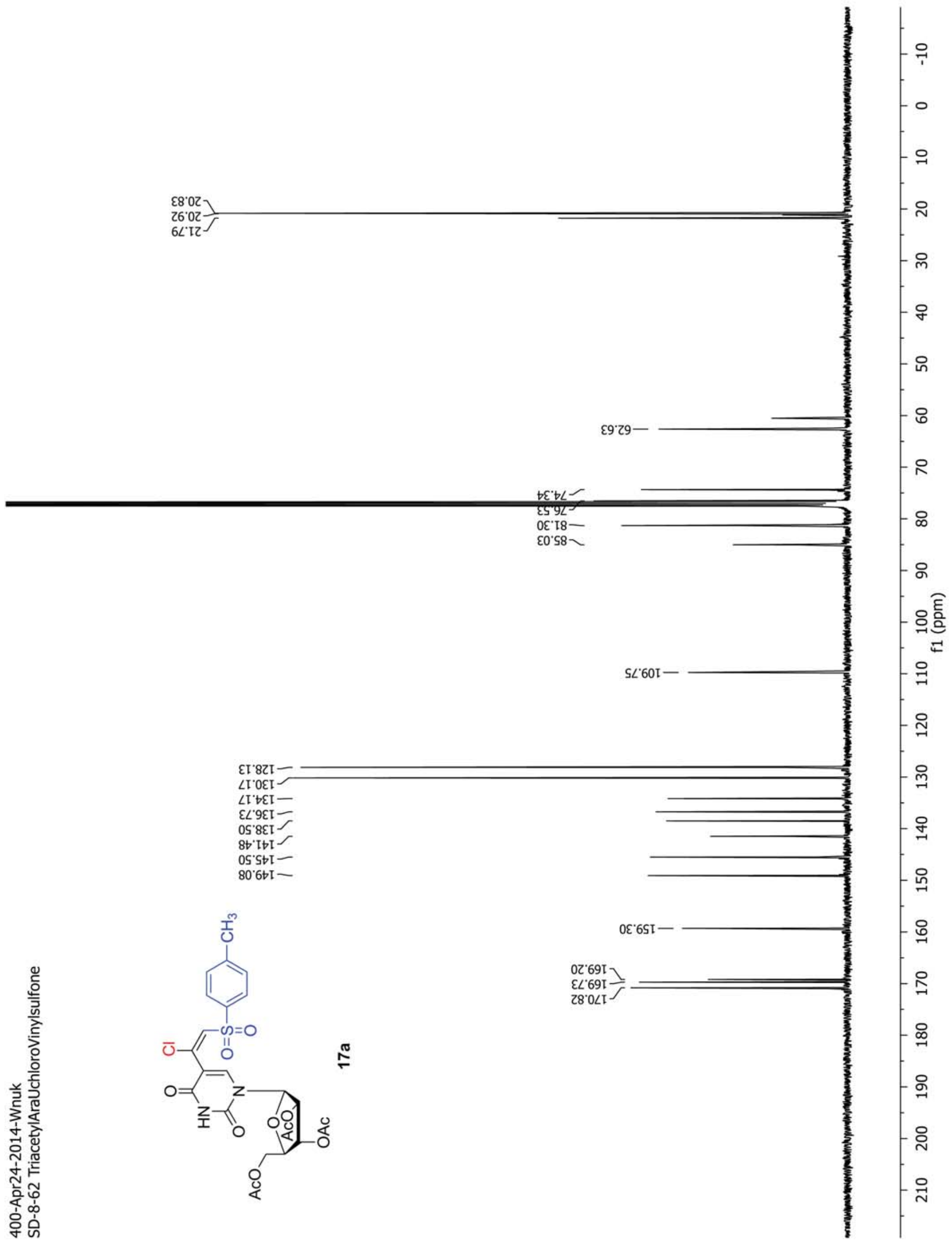


Page |S32

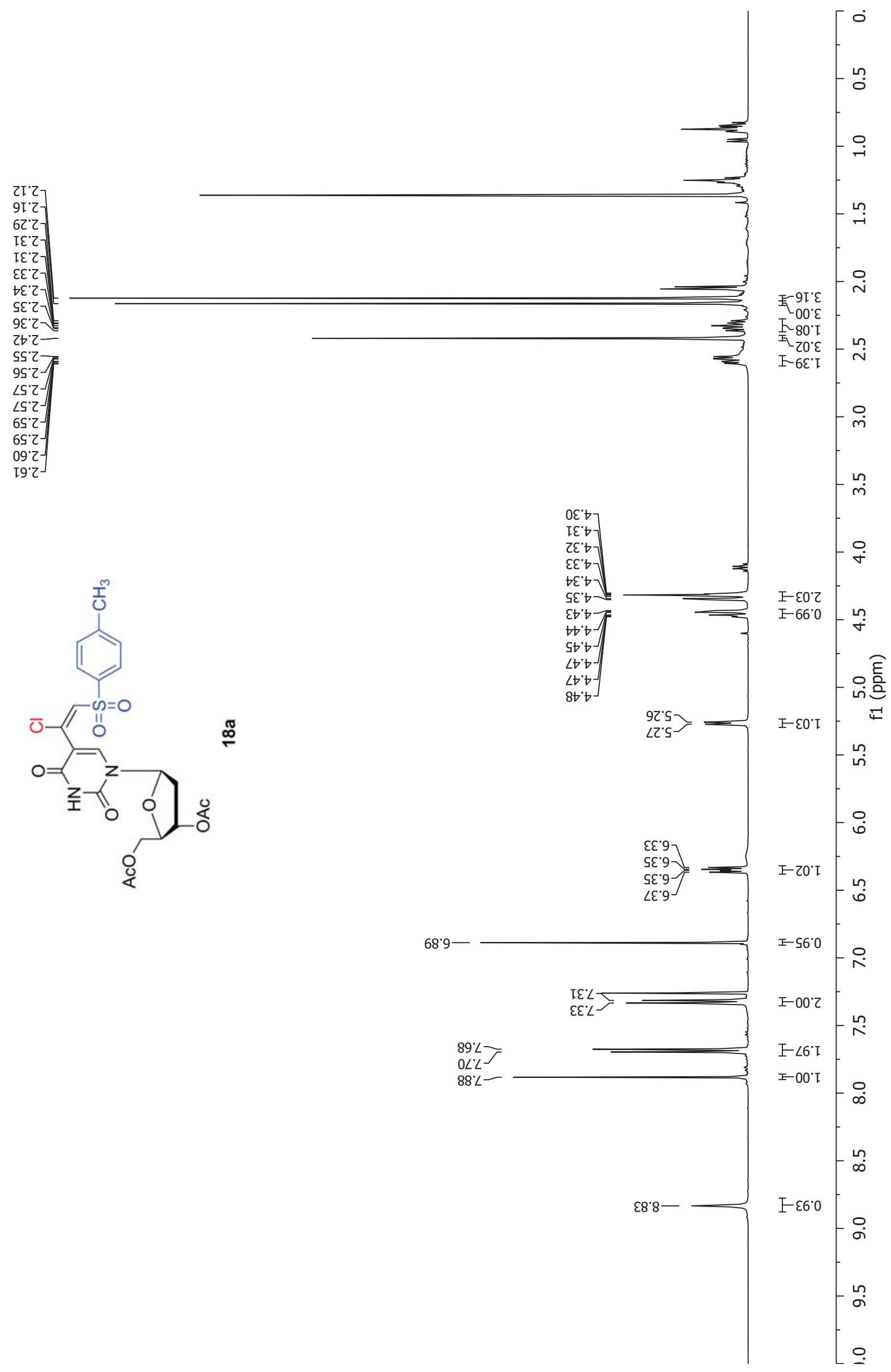


Page |S33

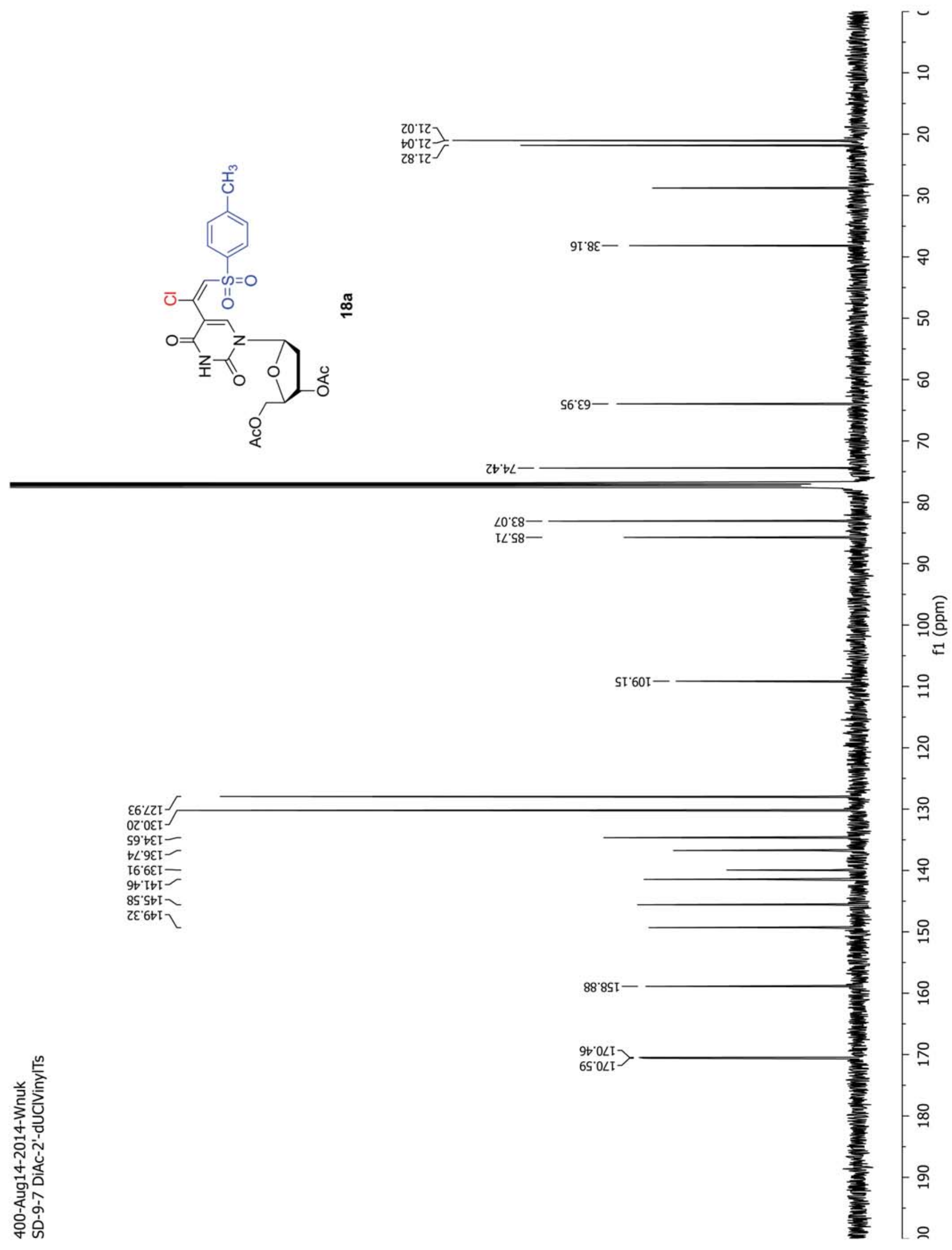


Page |S34

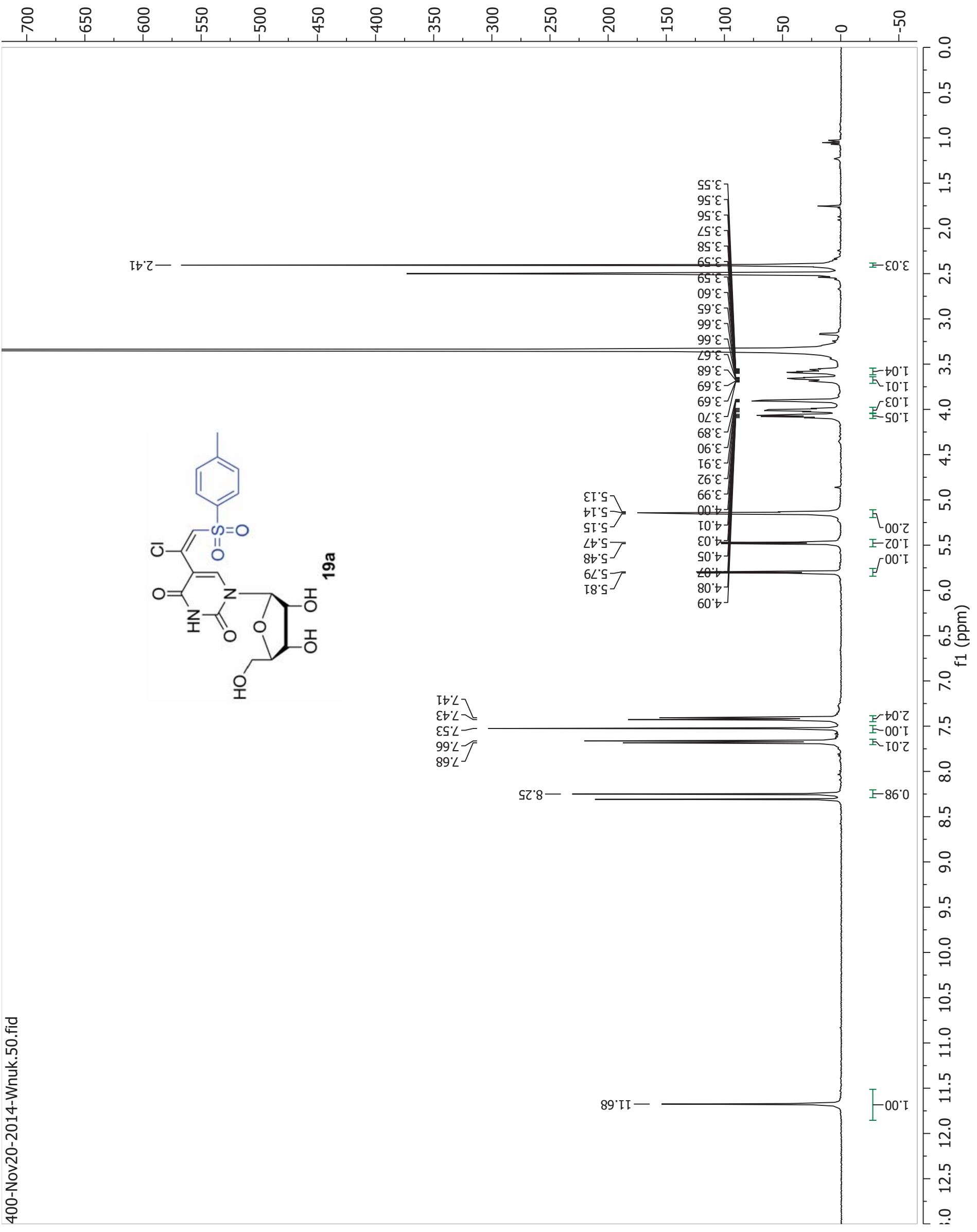


Page |S35

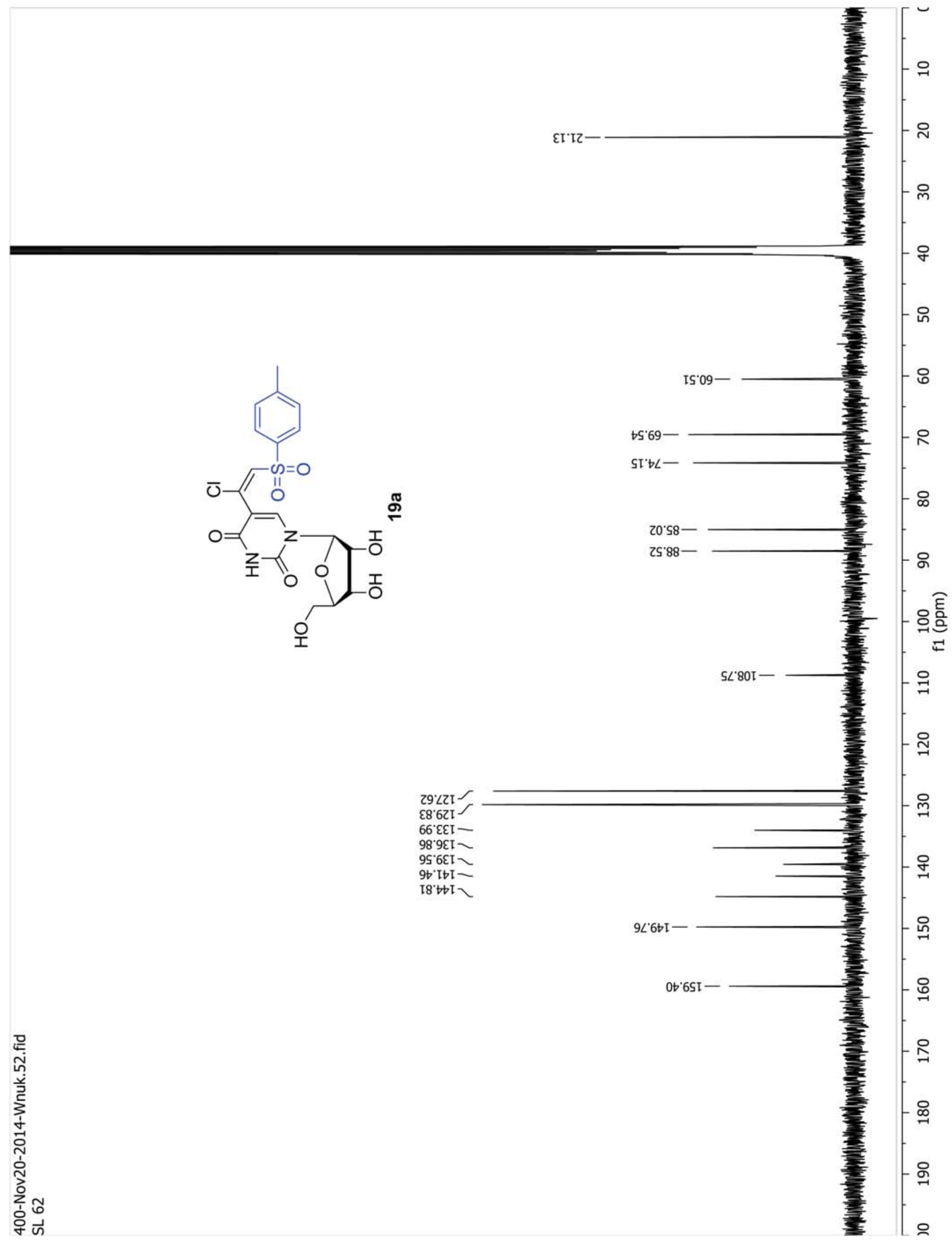


Page |S36

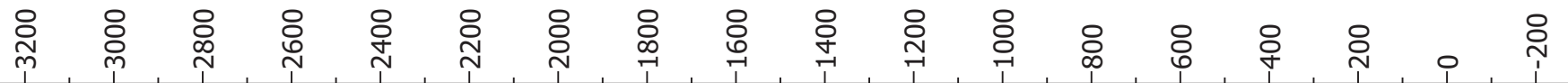

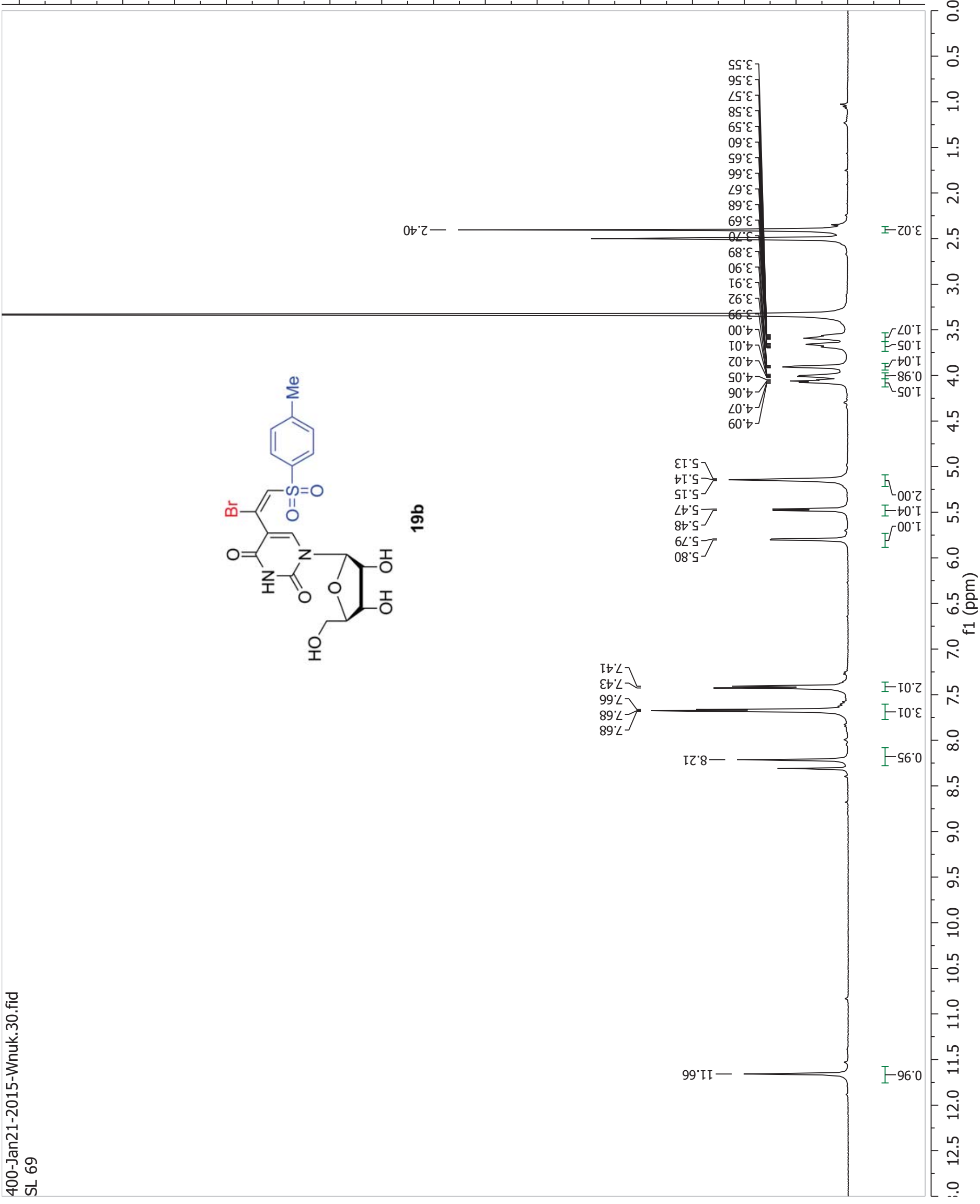


Page |S37

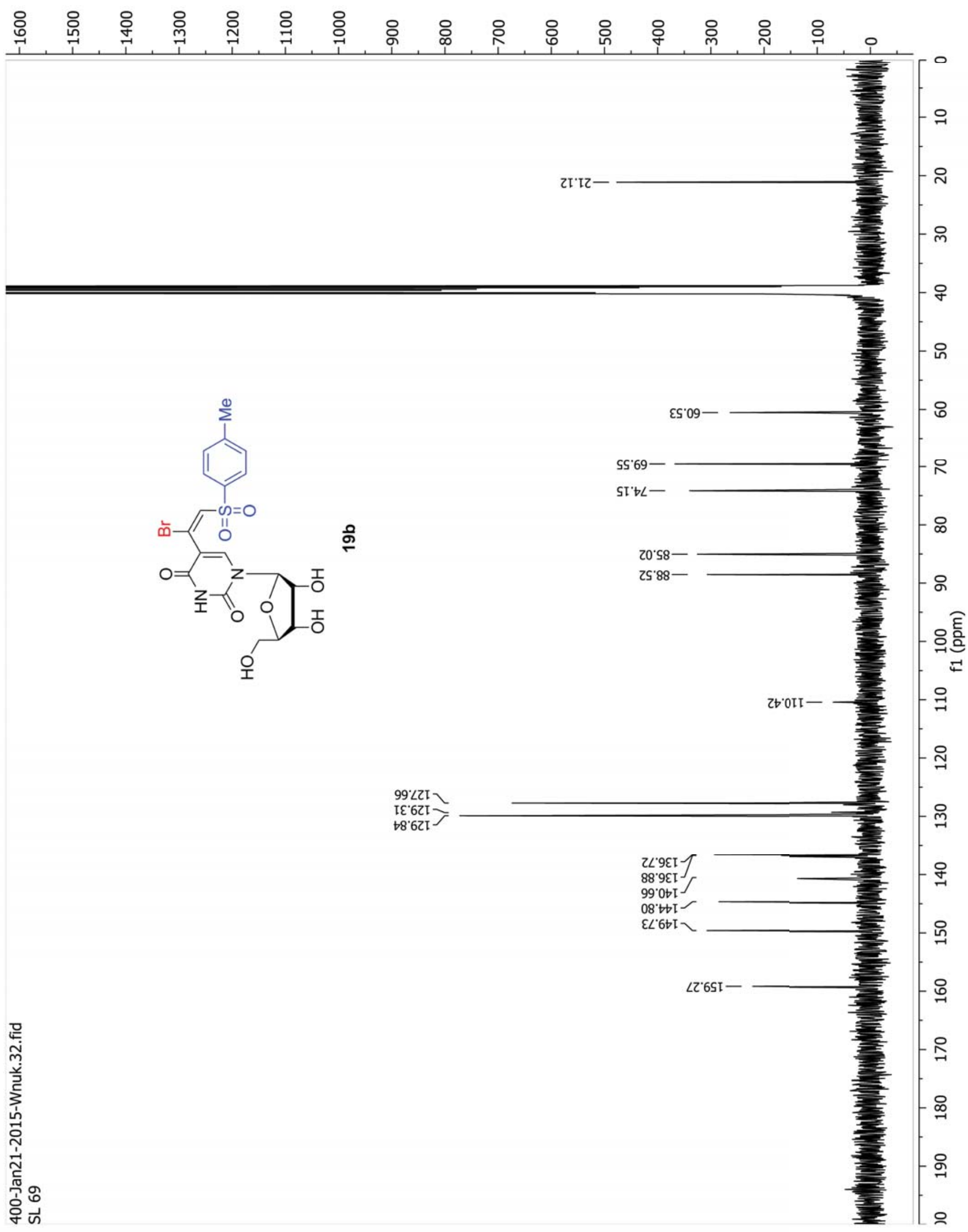


Page |S38

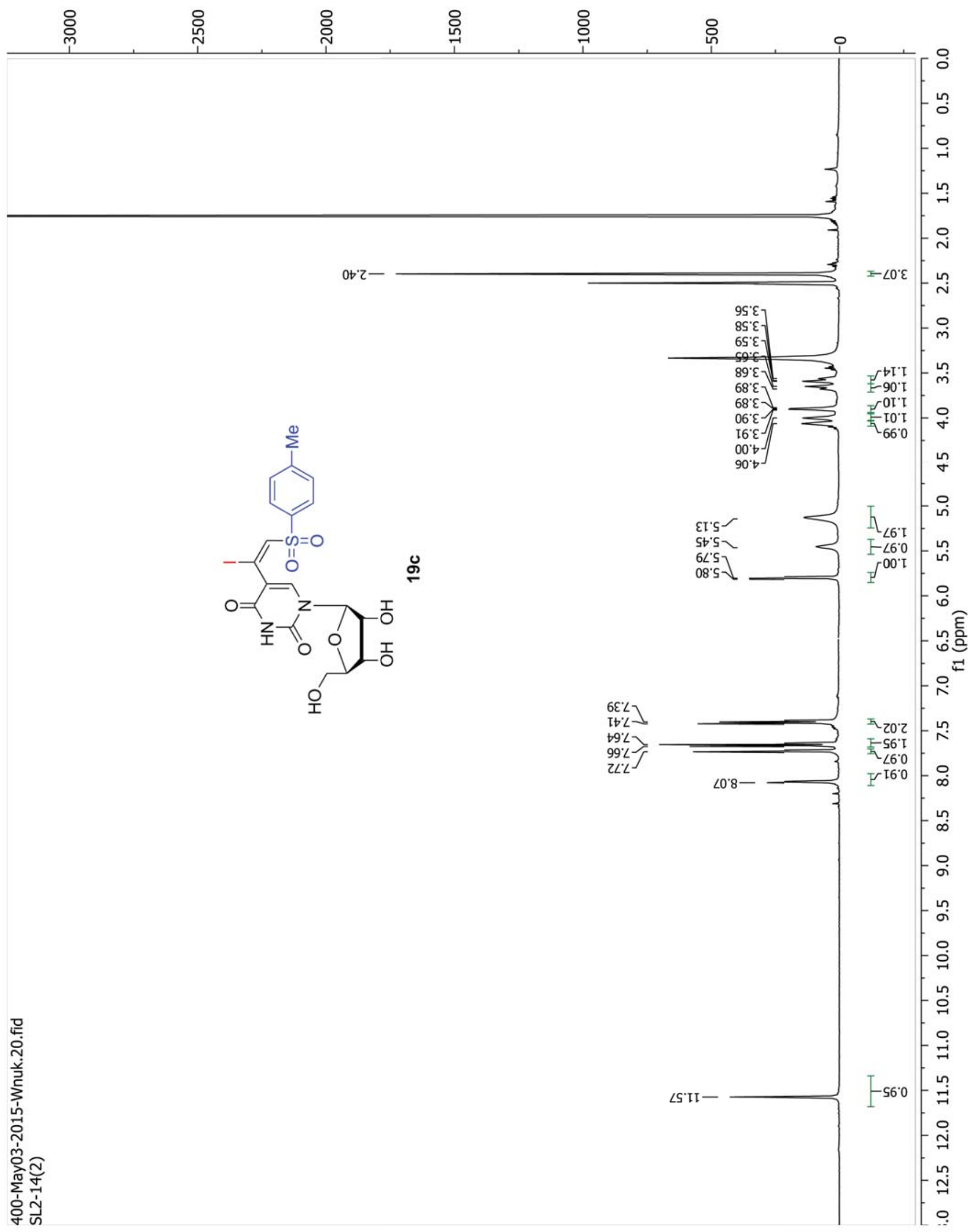


Page |S39

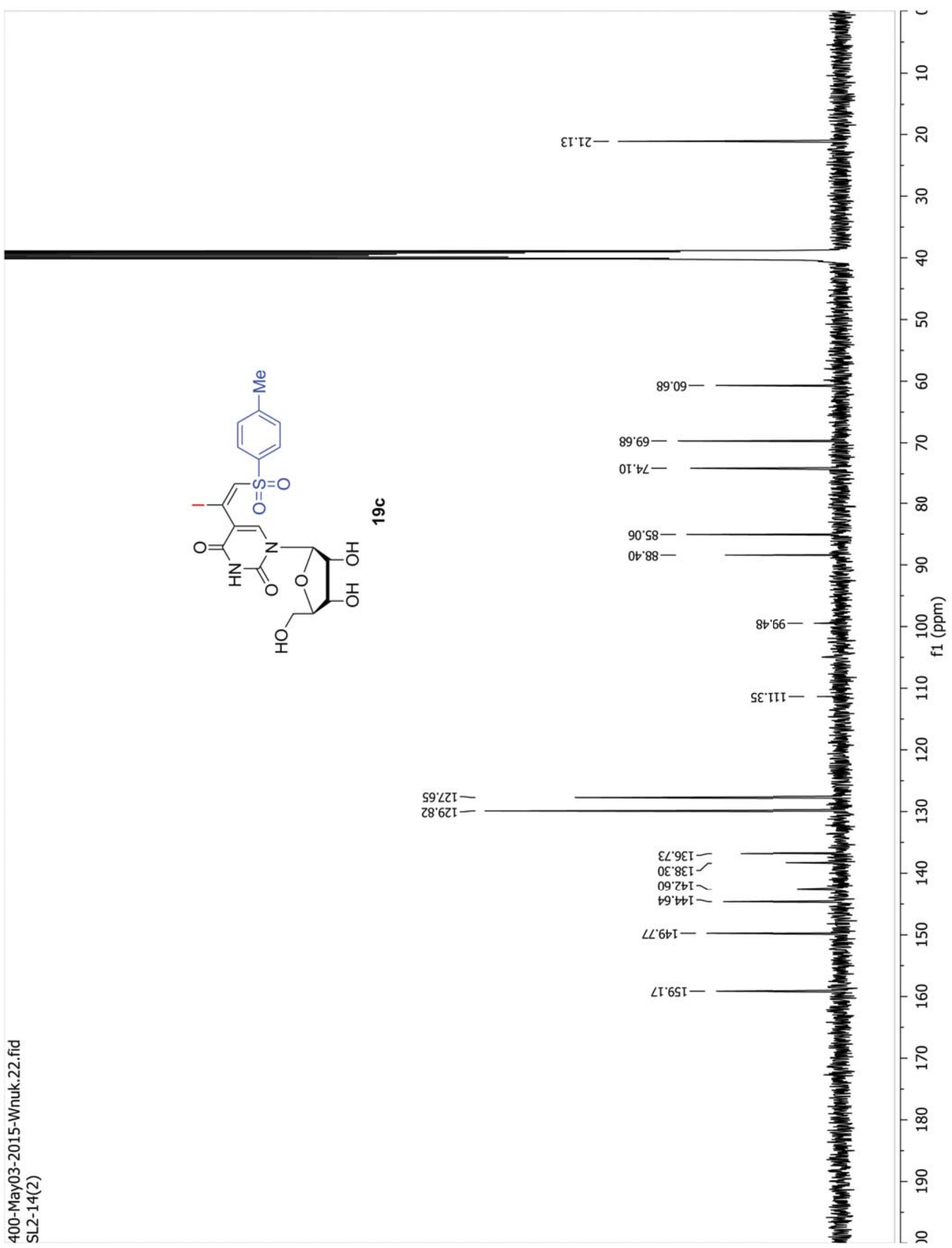


Page |S40

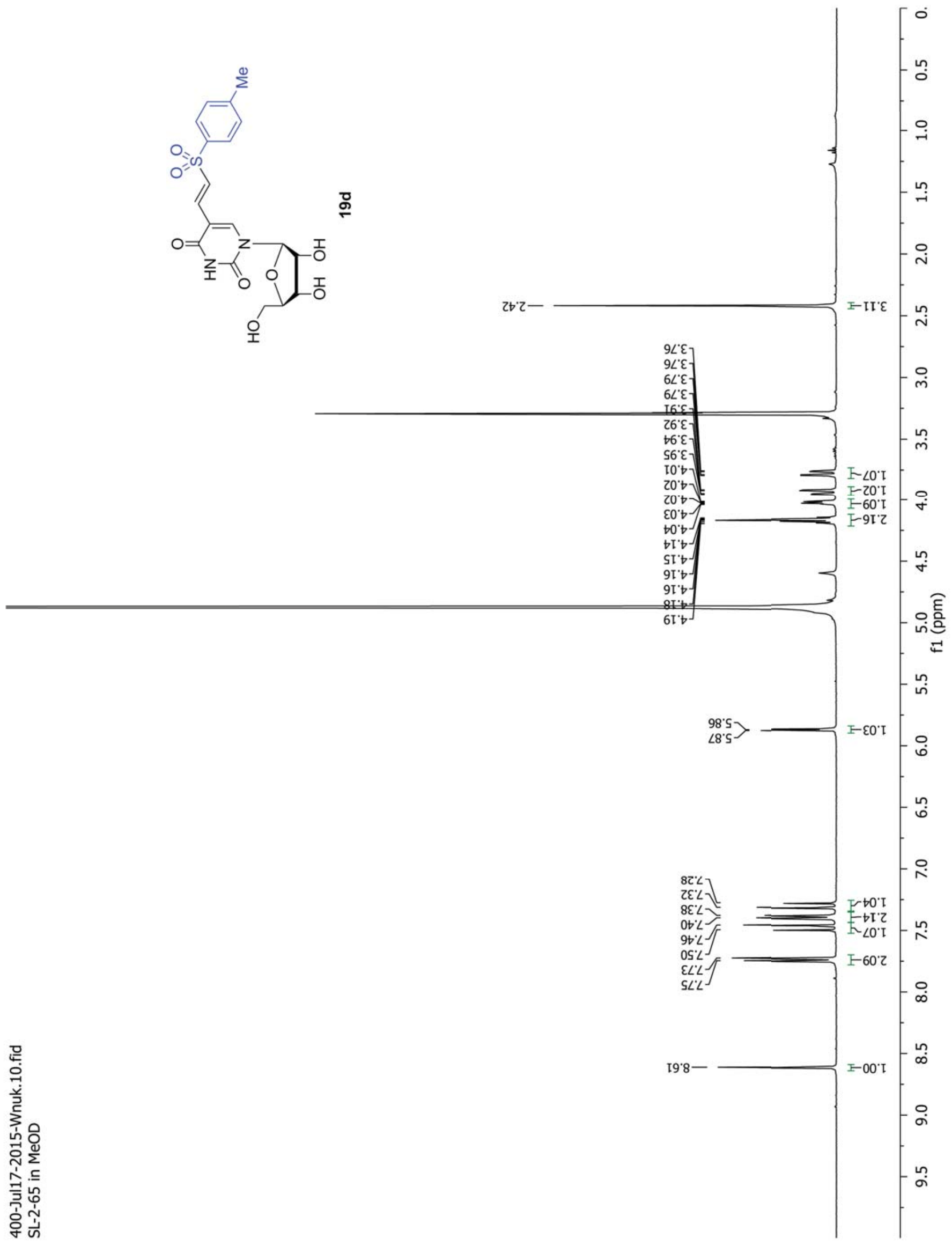


Page |S41

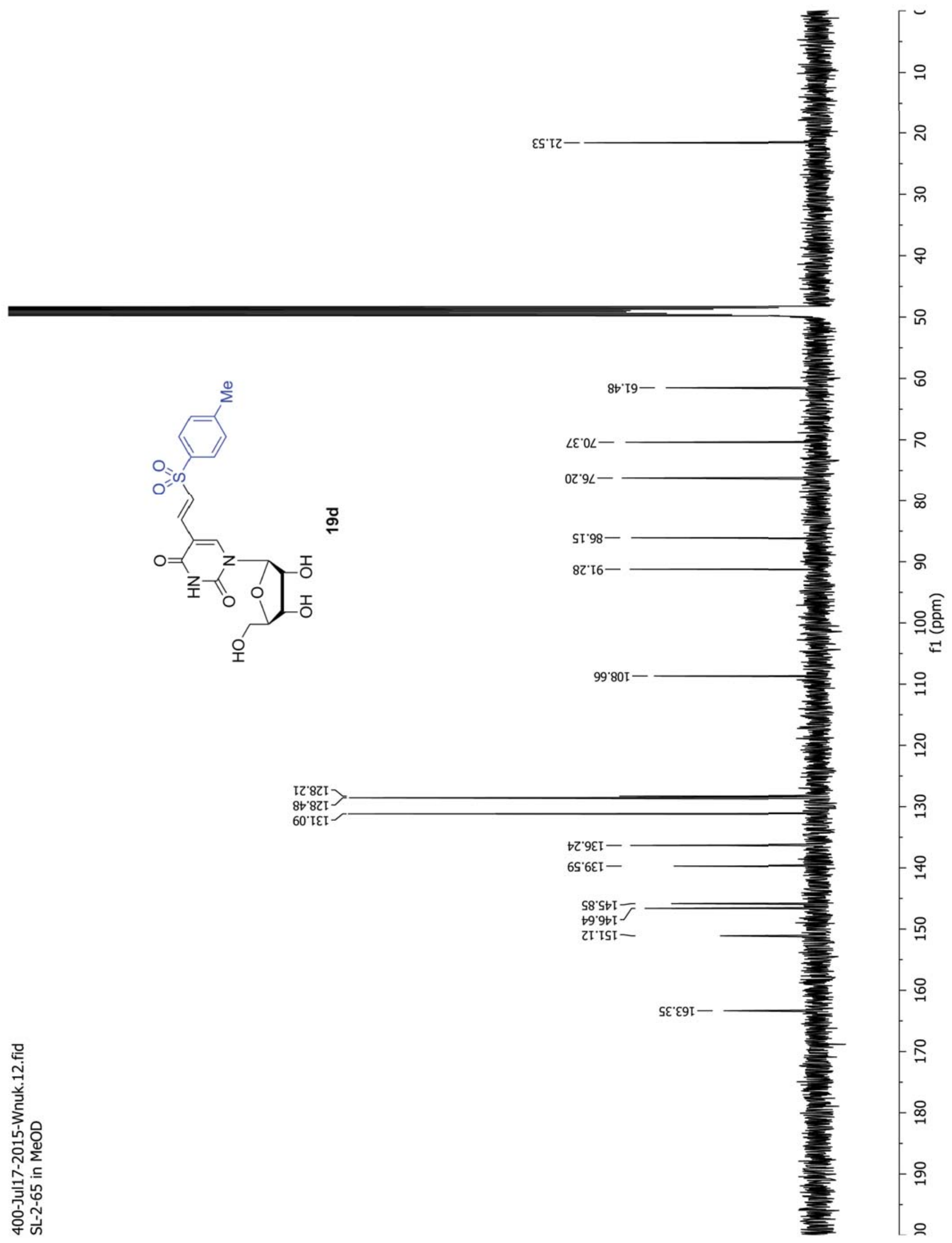


Page |S42

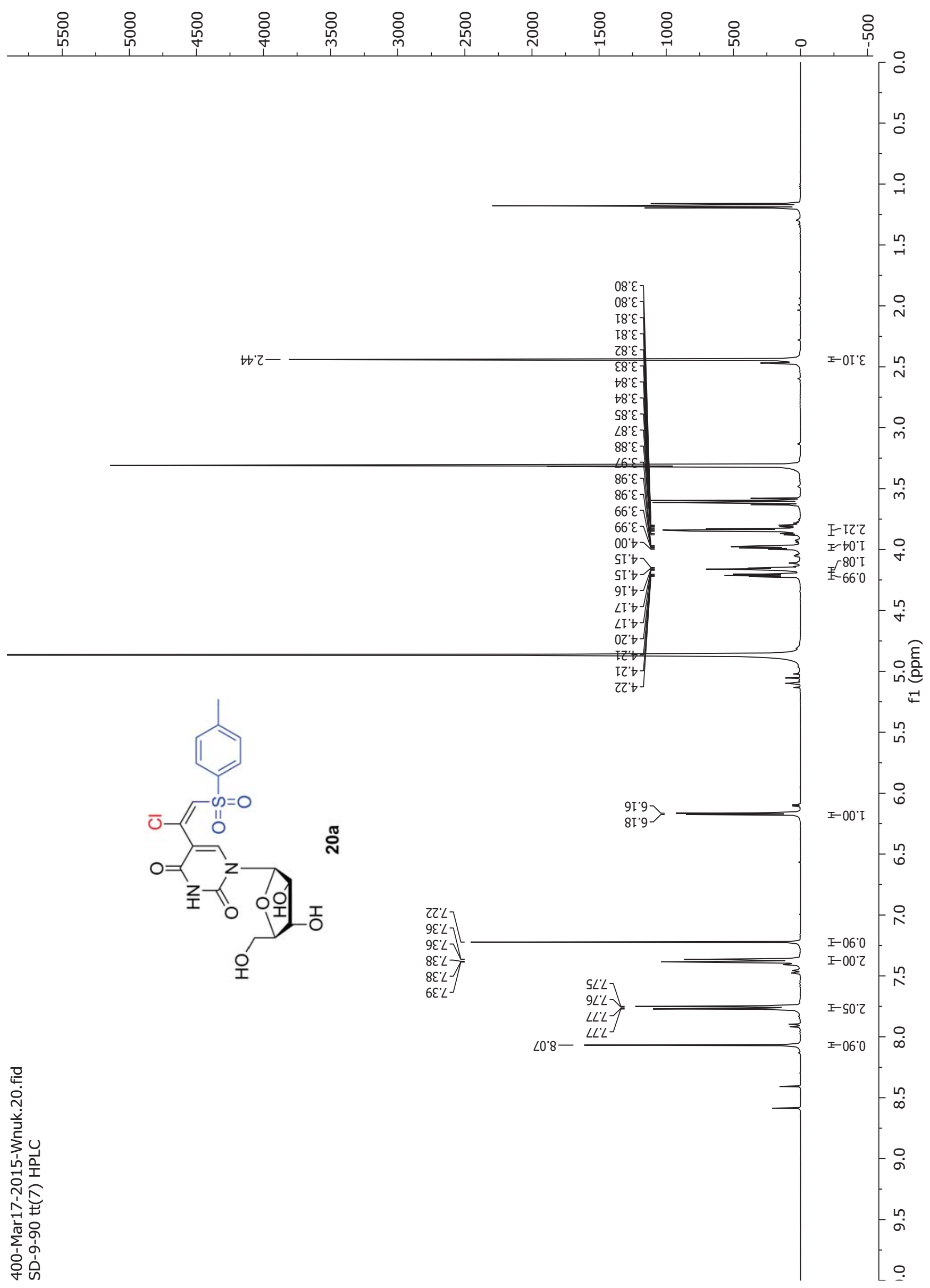


Page |S43
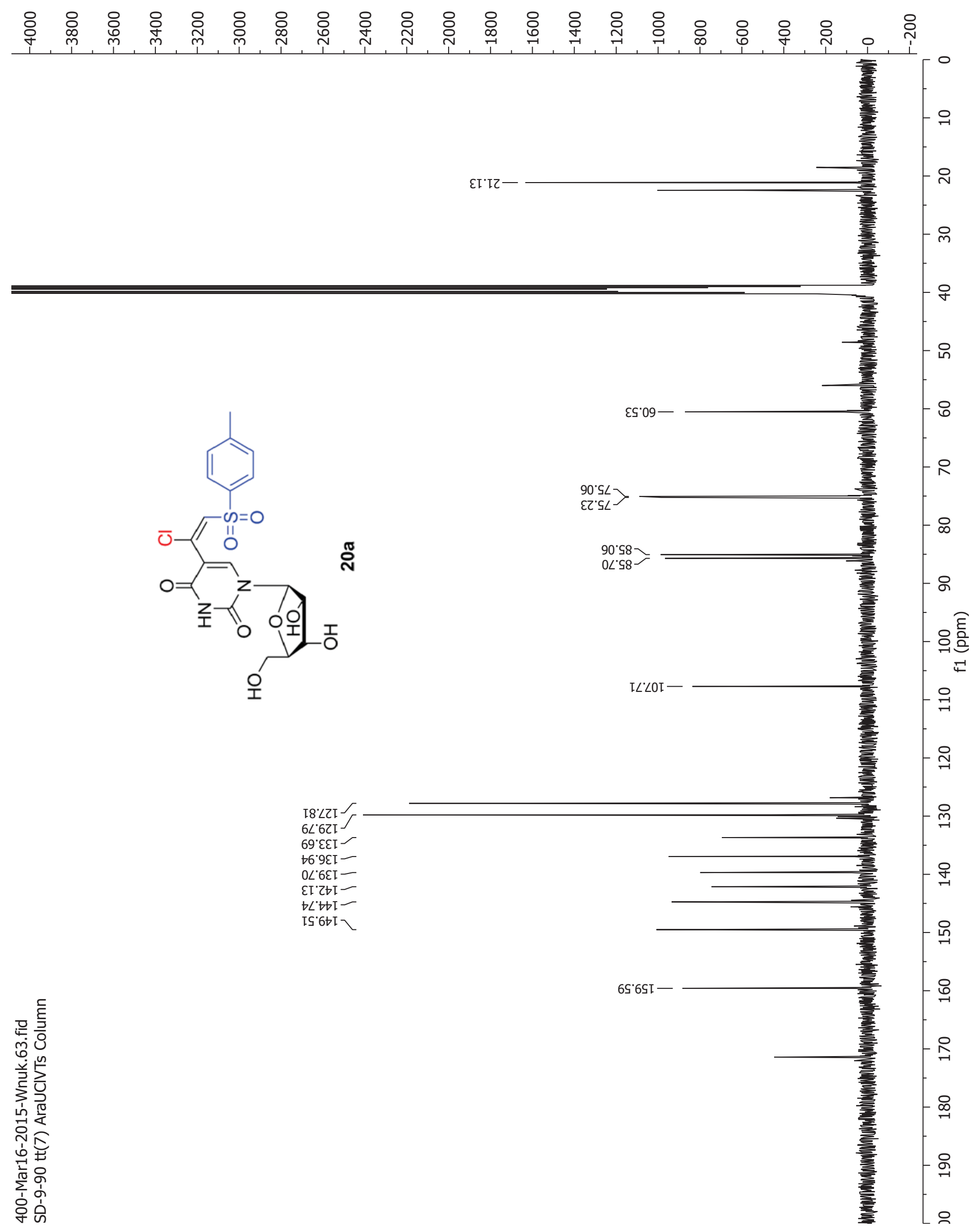
Page |S44

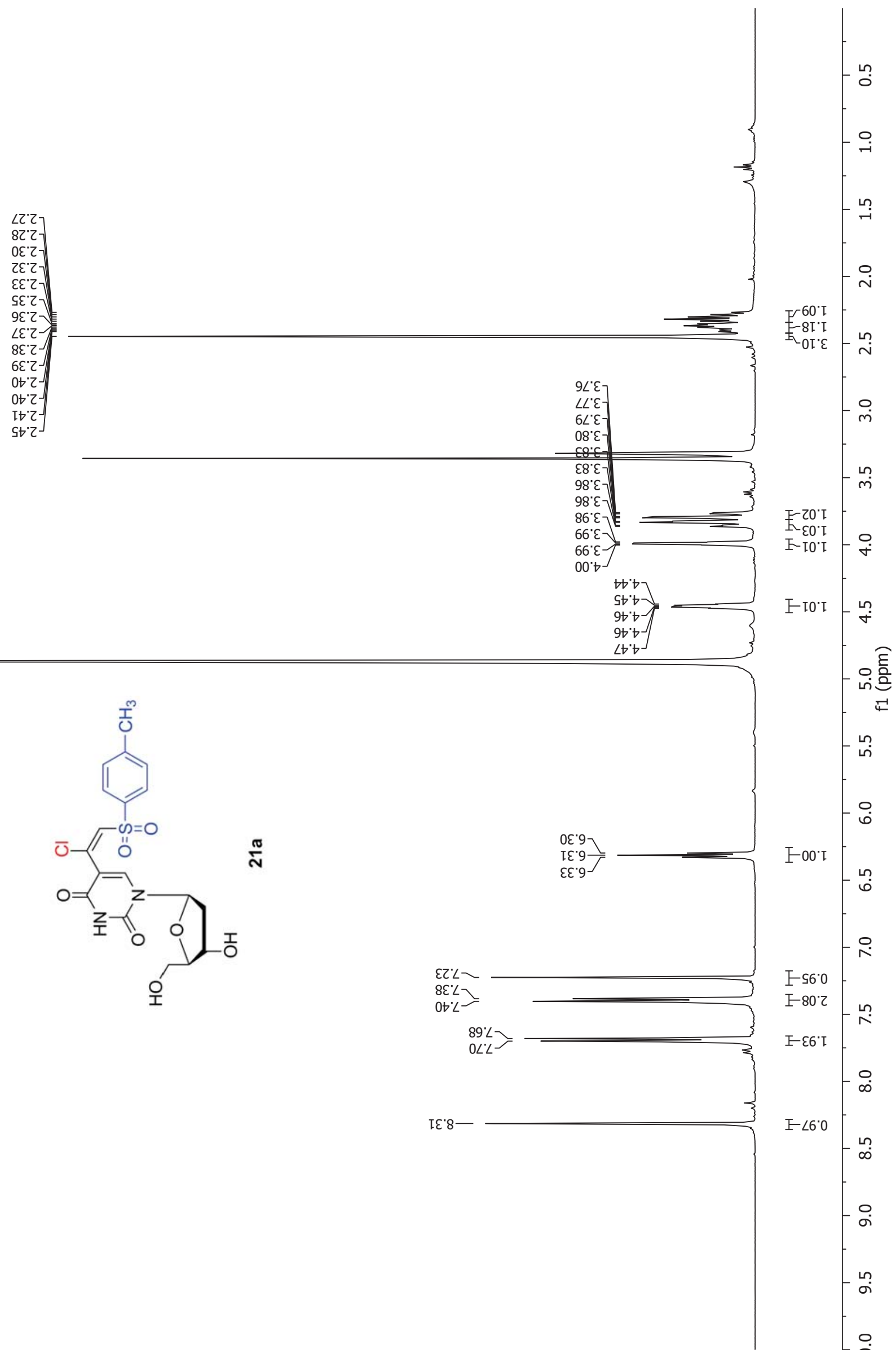


Page |S45

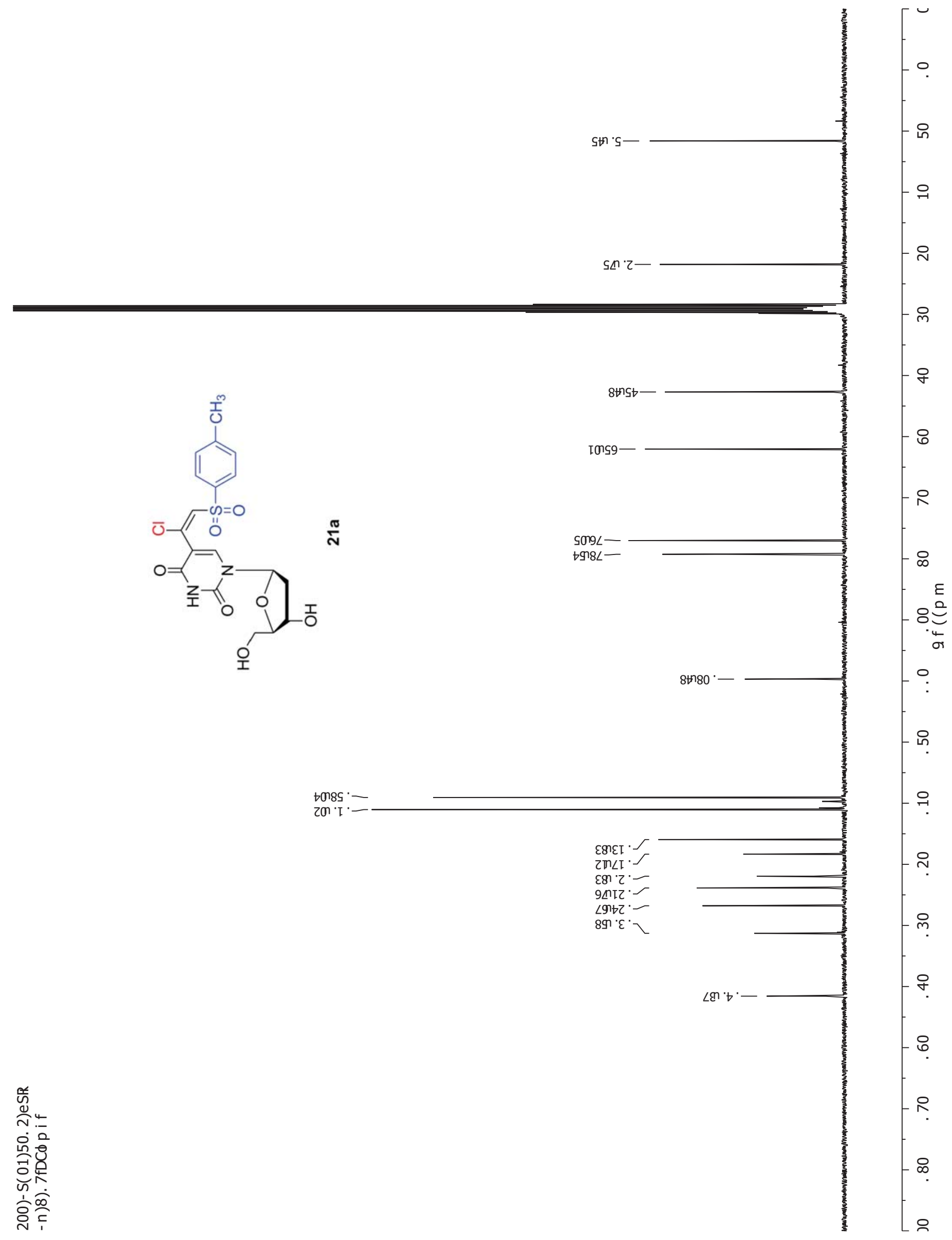


Page |S46

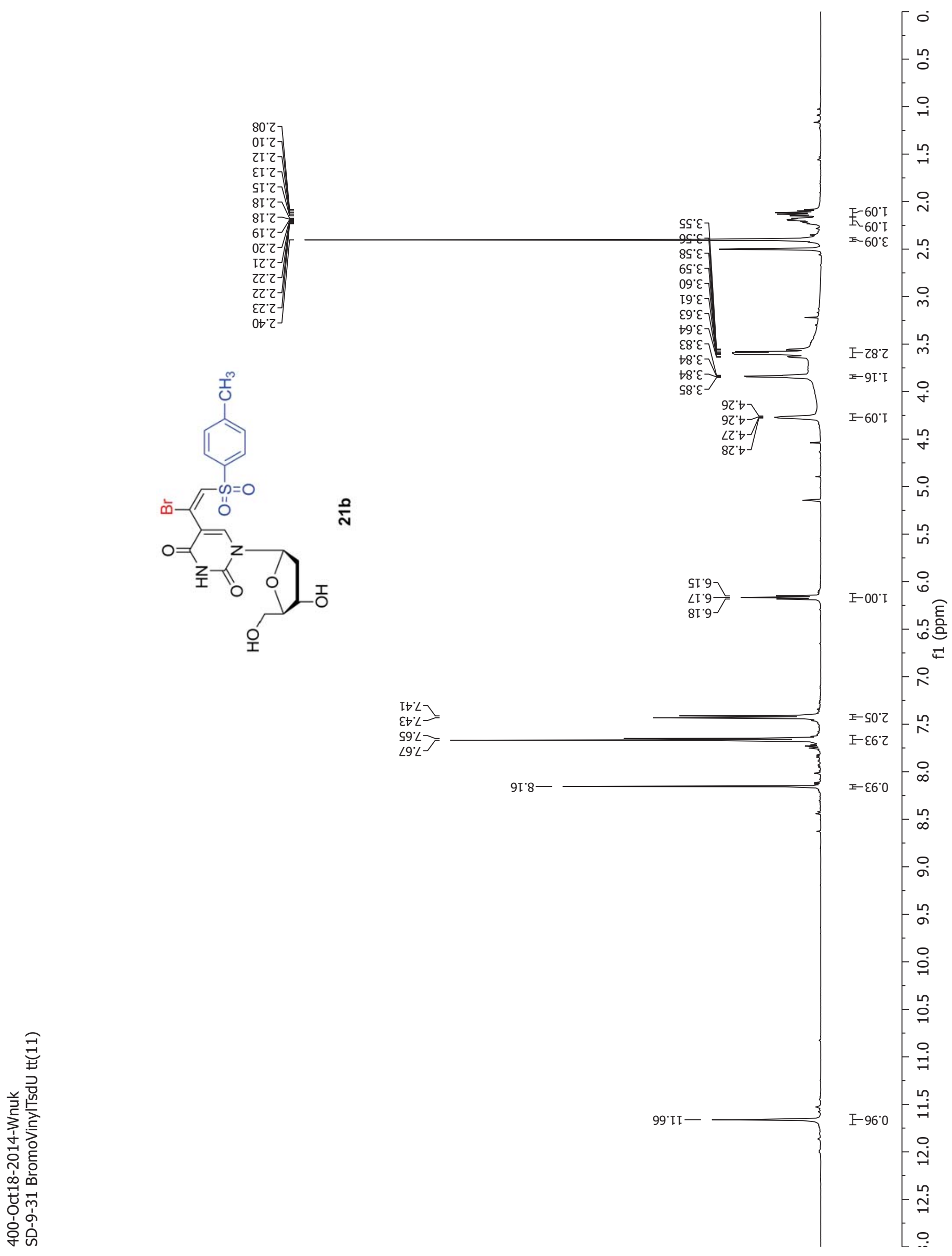


Page |S47

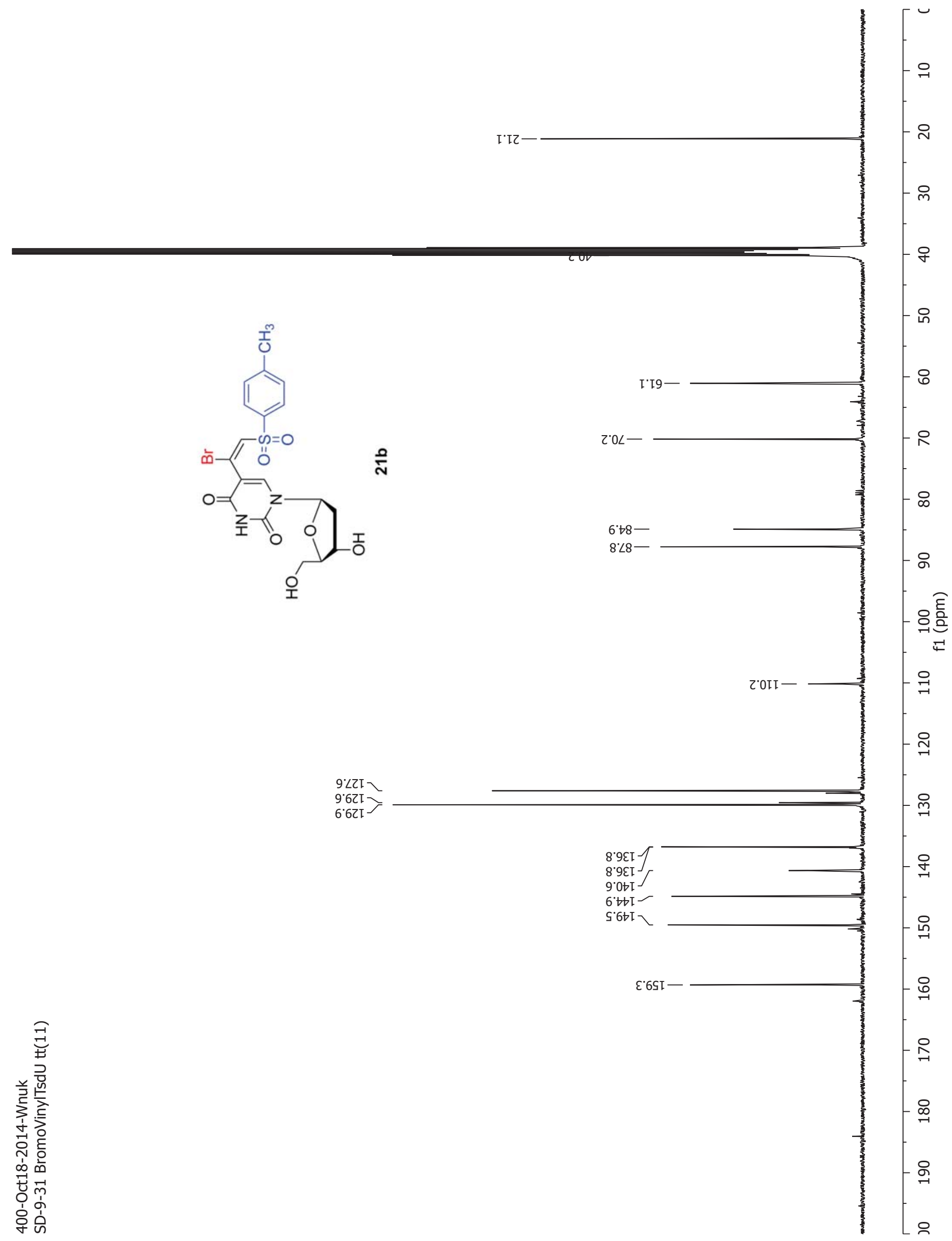


Page |S48
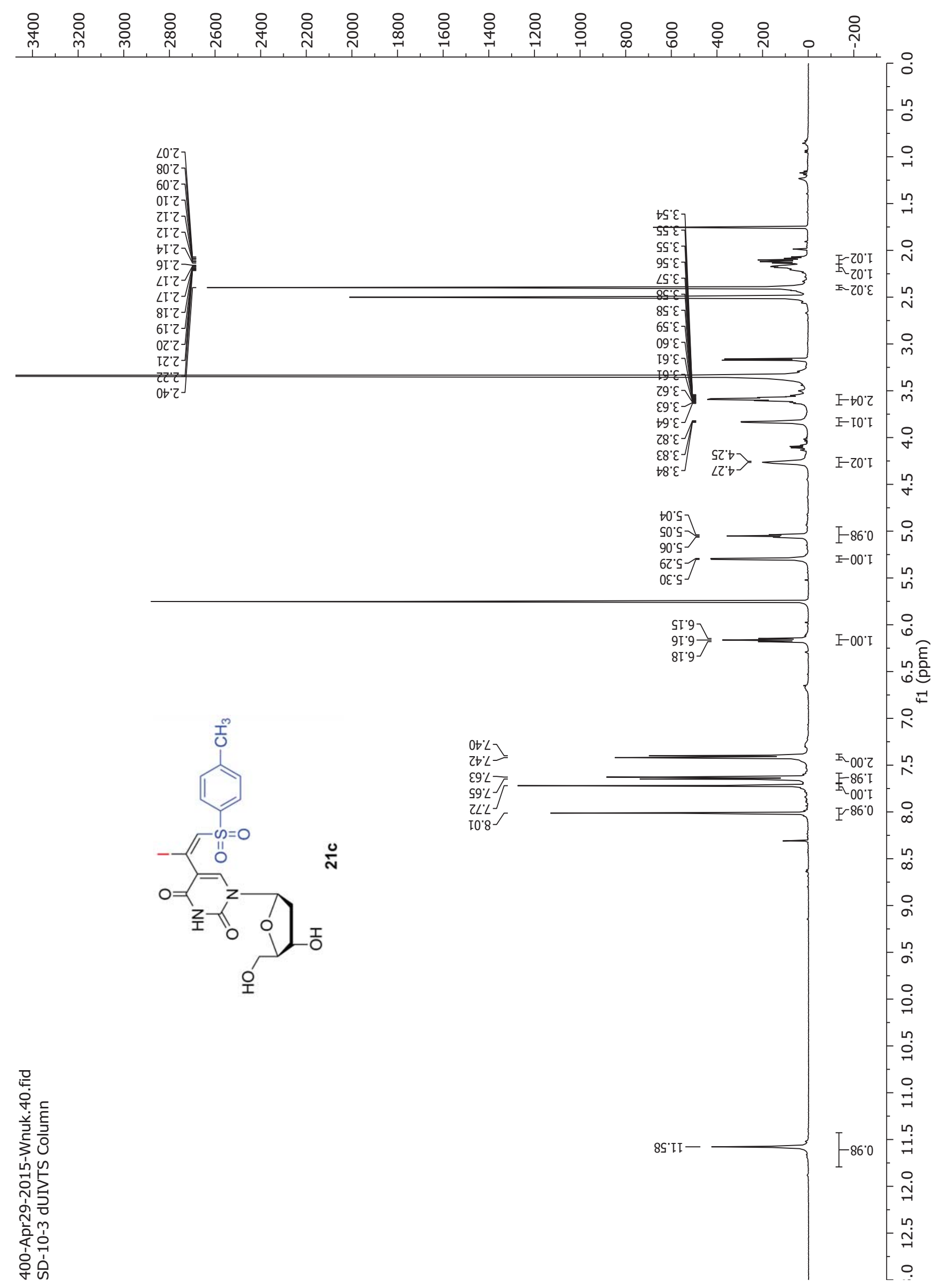
Page |S49

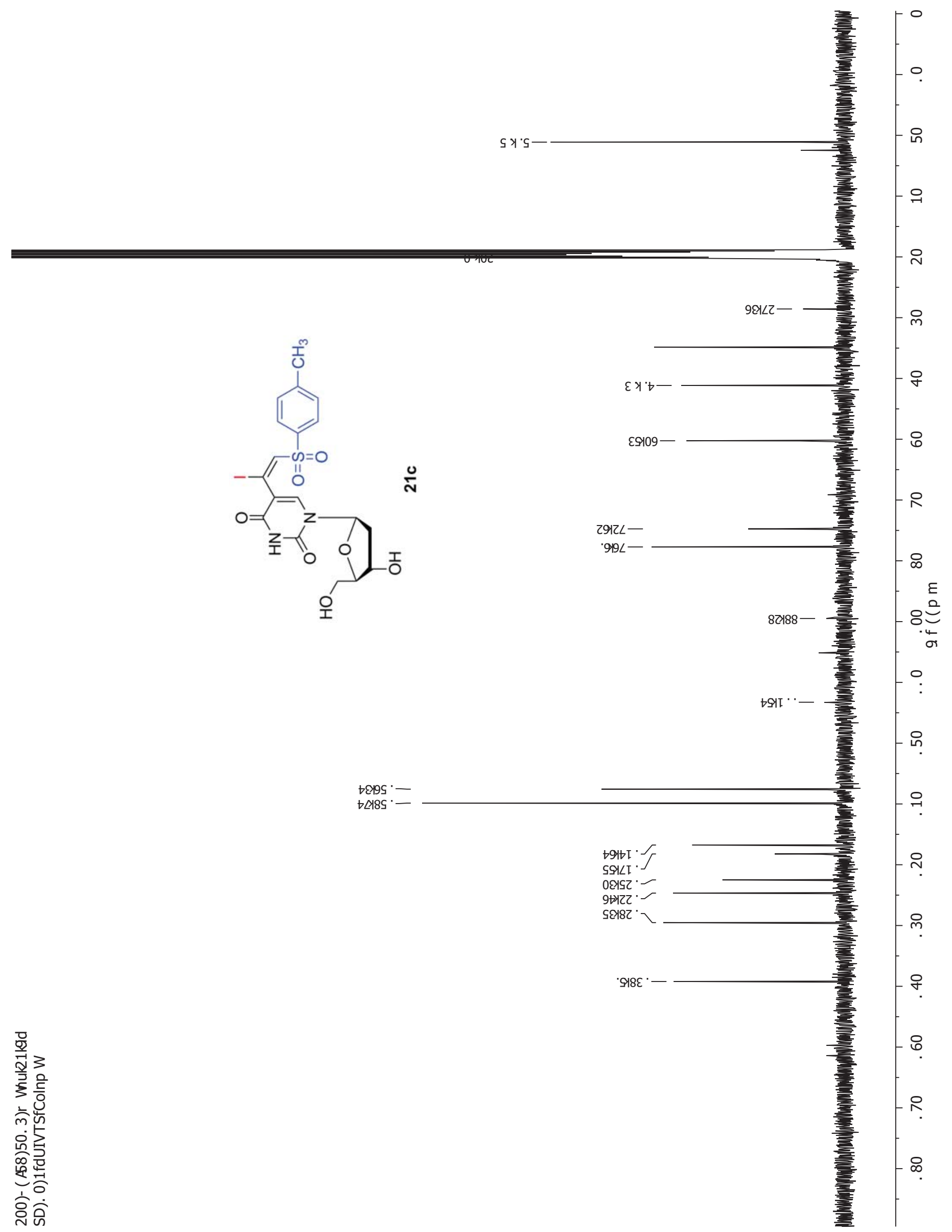


Page |S50

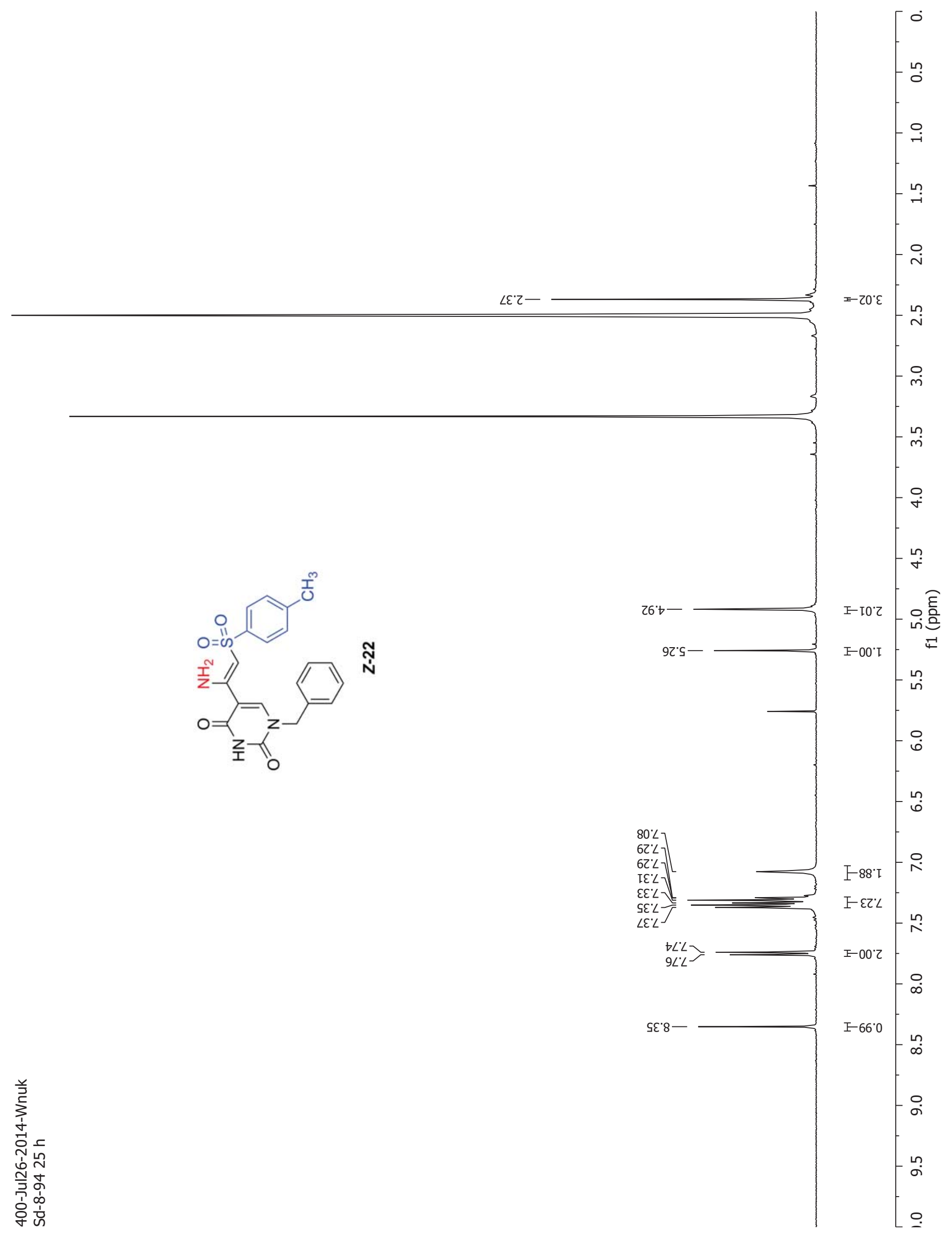


Page |S51

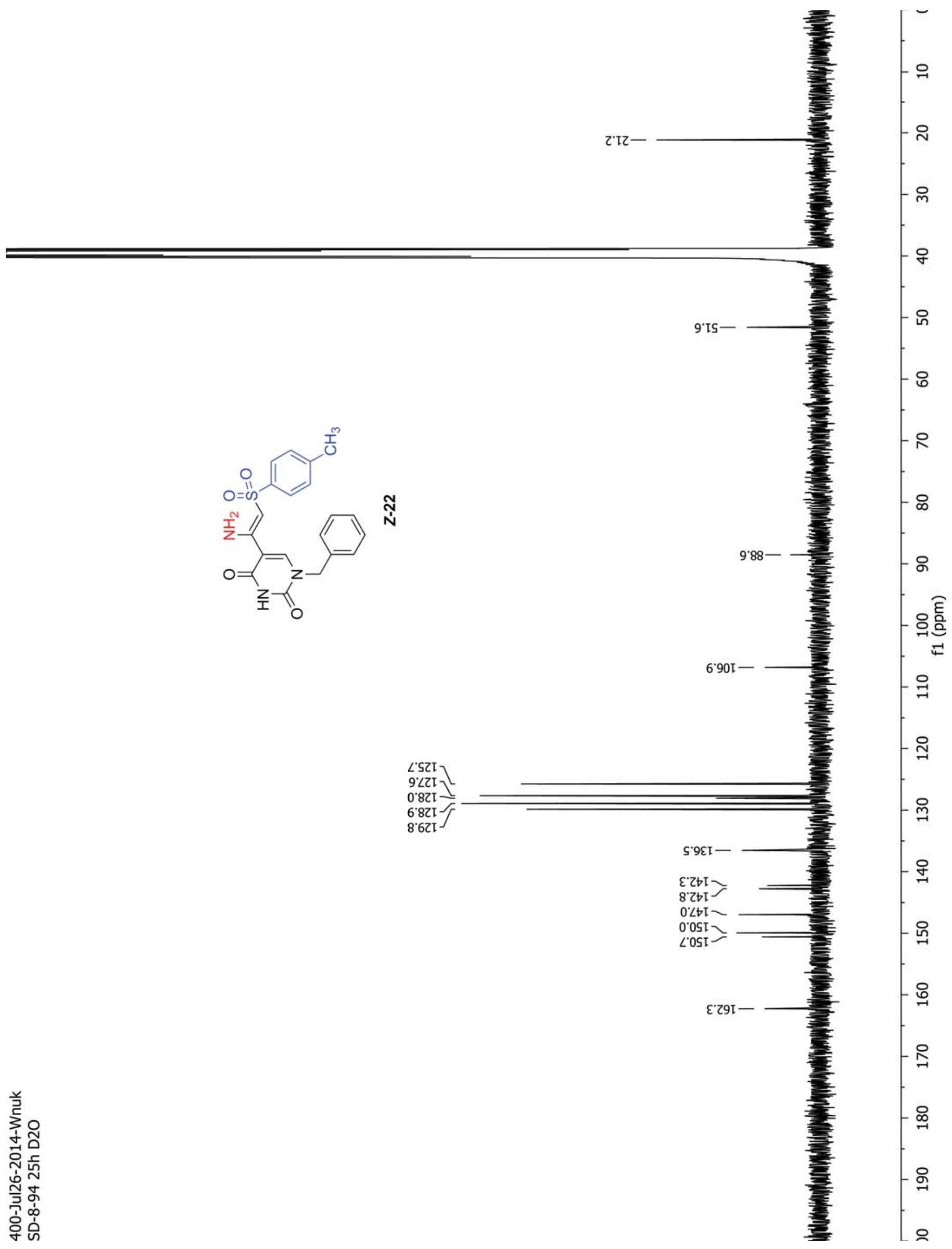


Page |S52

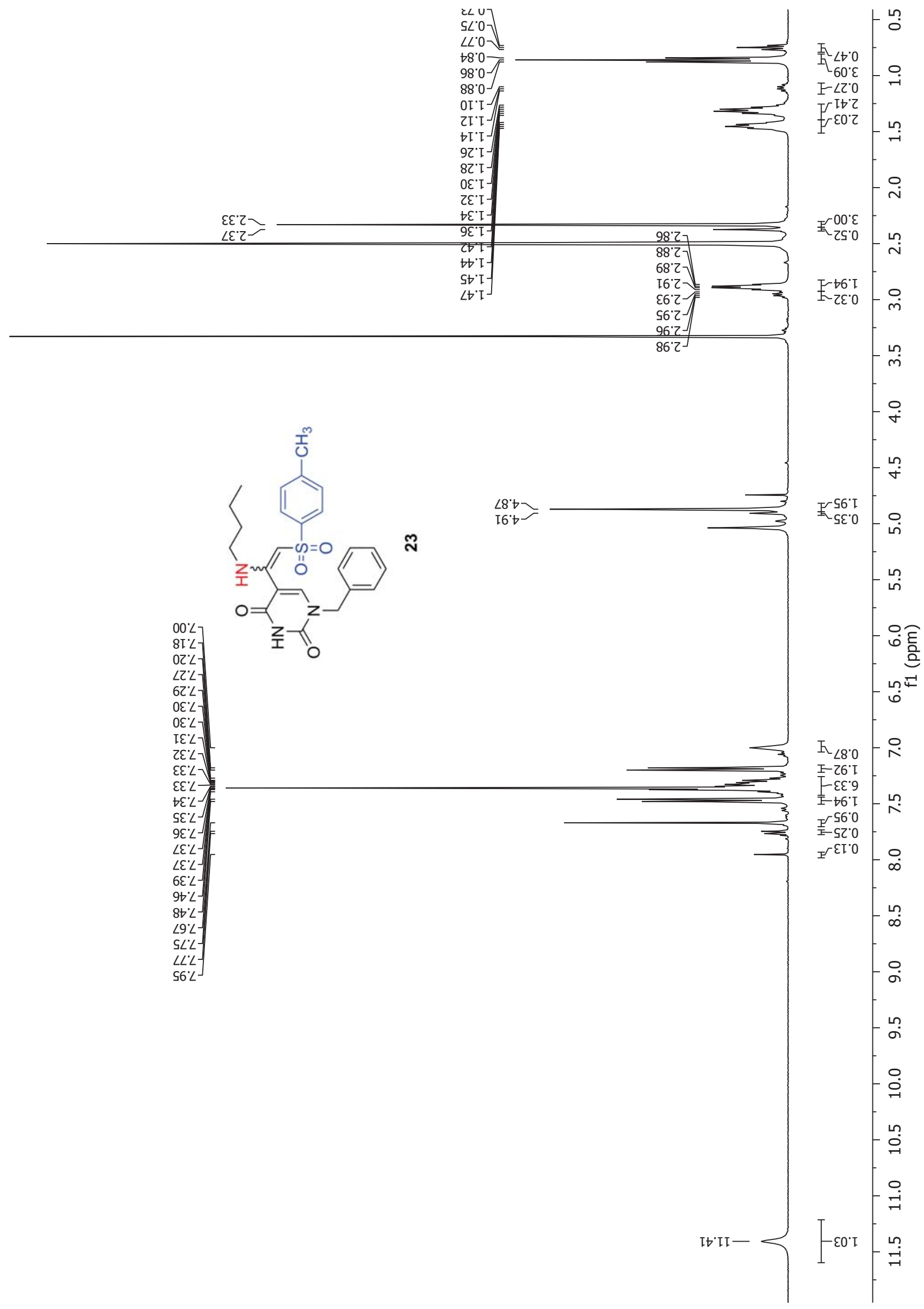


Page |S53

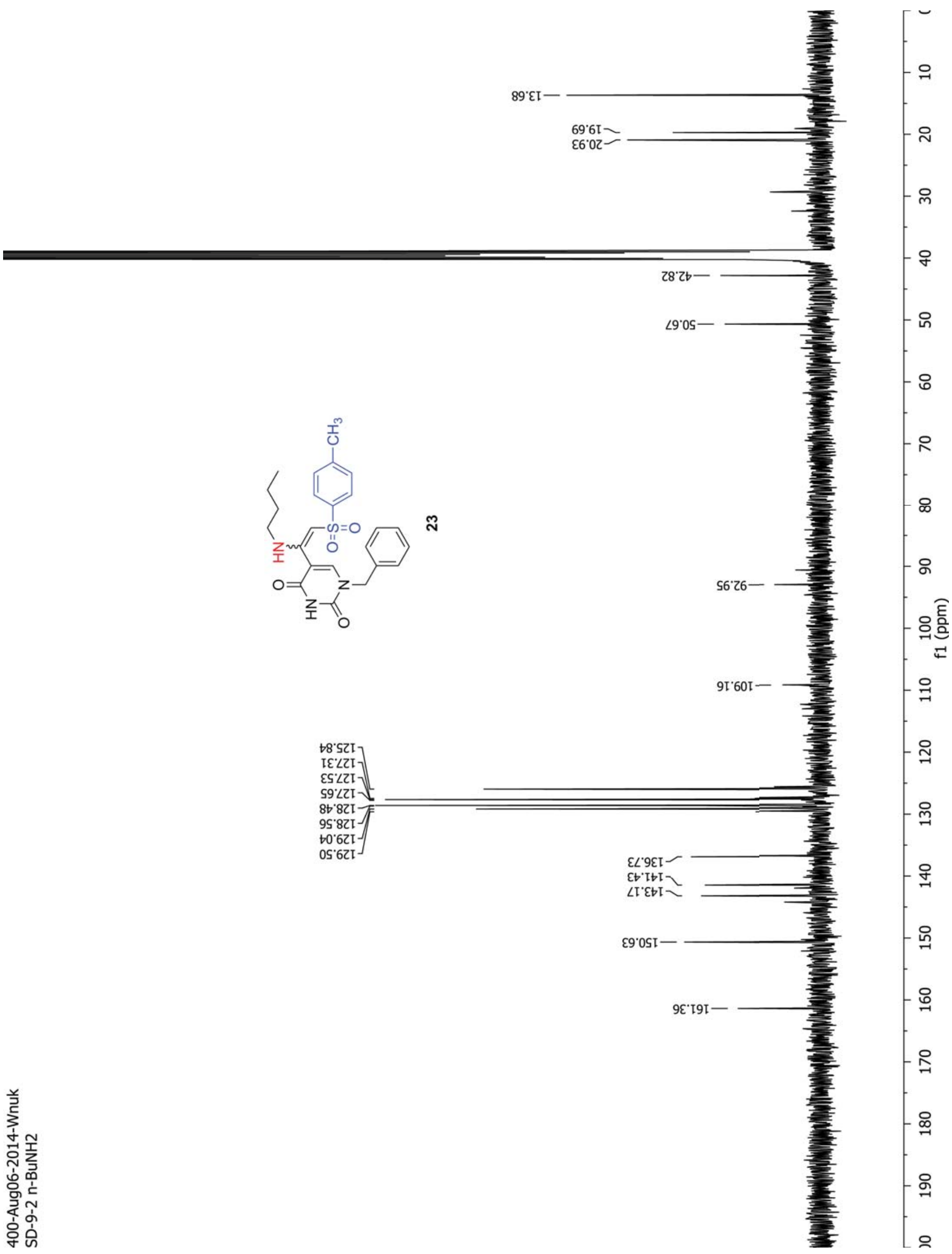


Page |S54

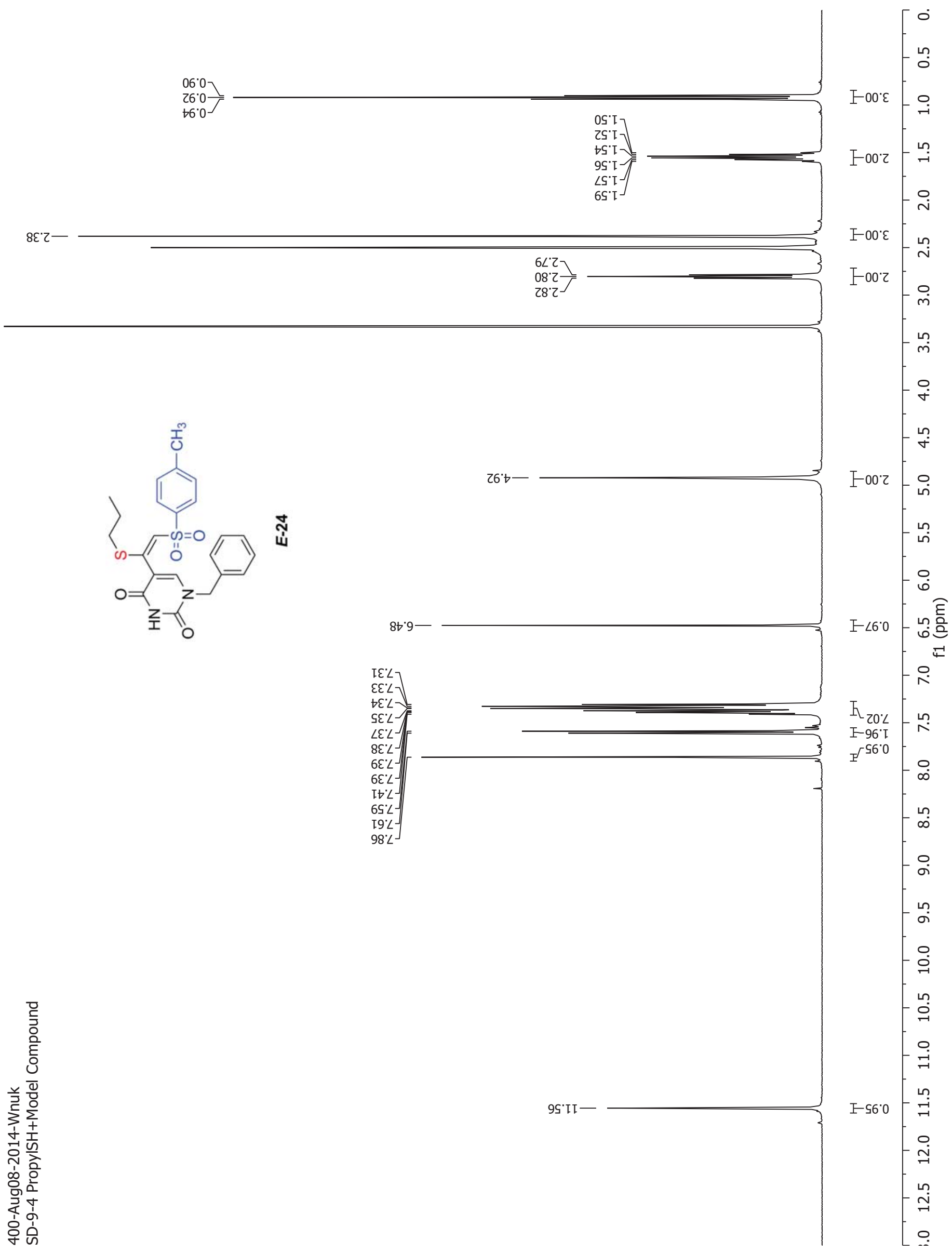


Page |S55

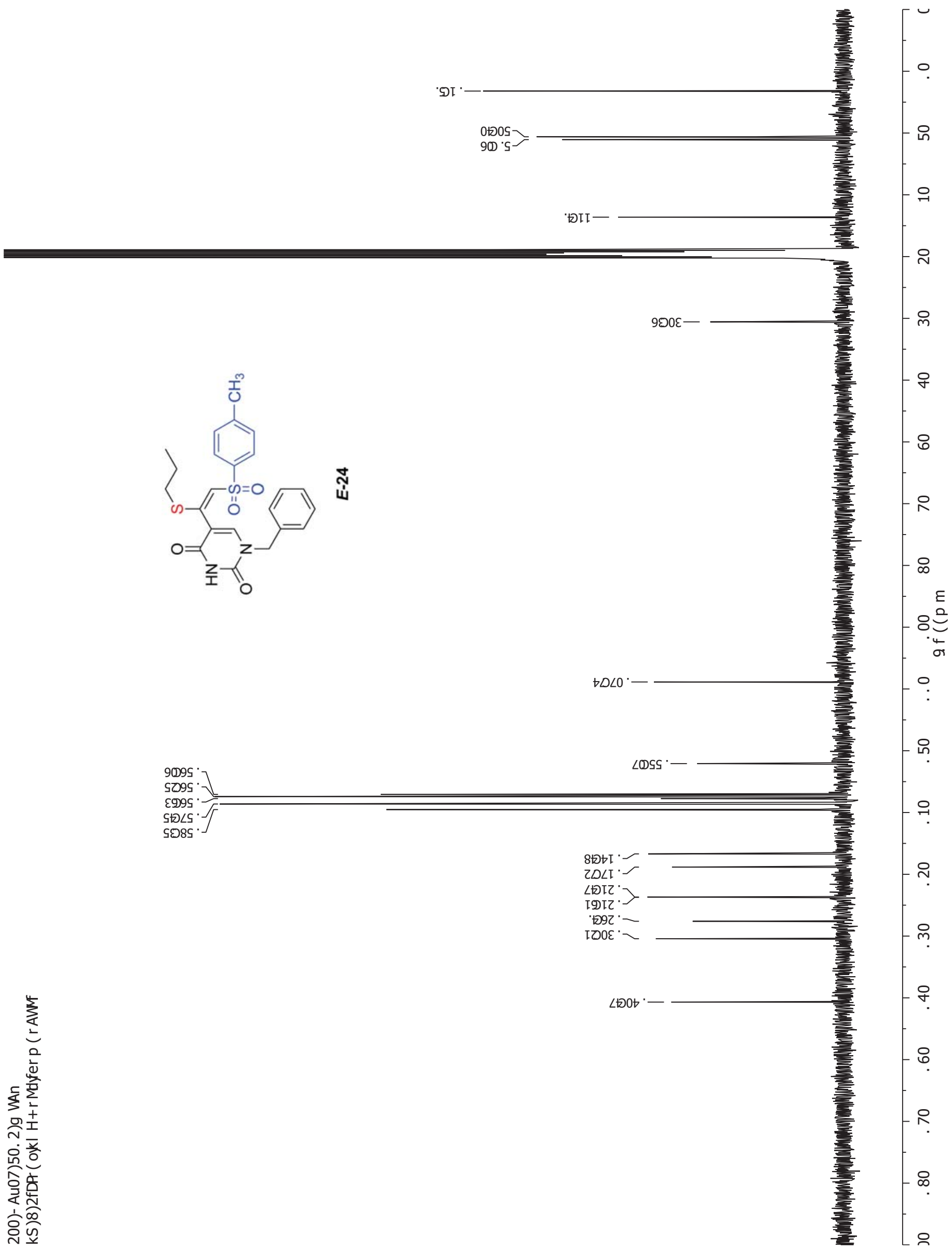


Page |S56

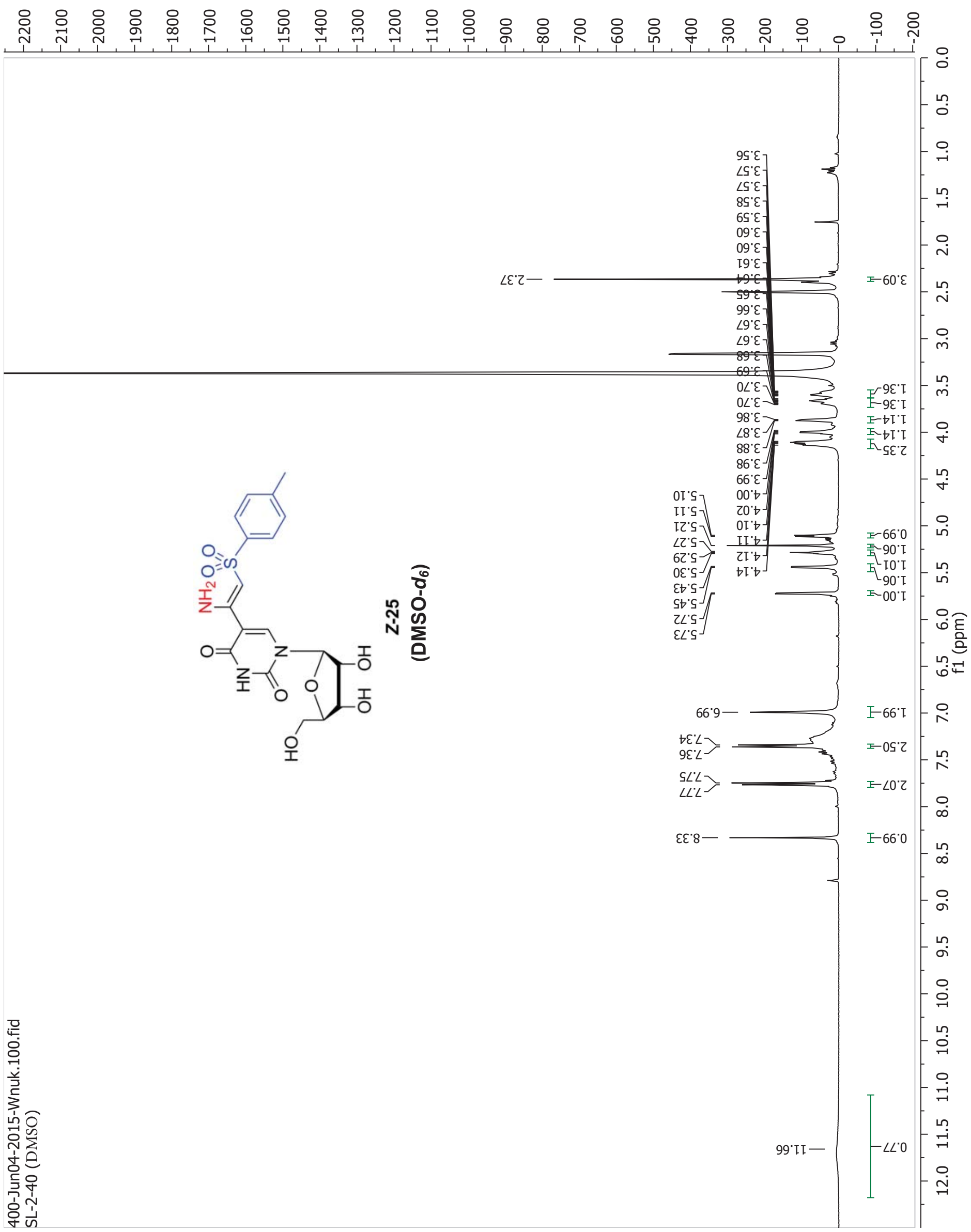


Page |S57

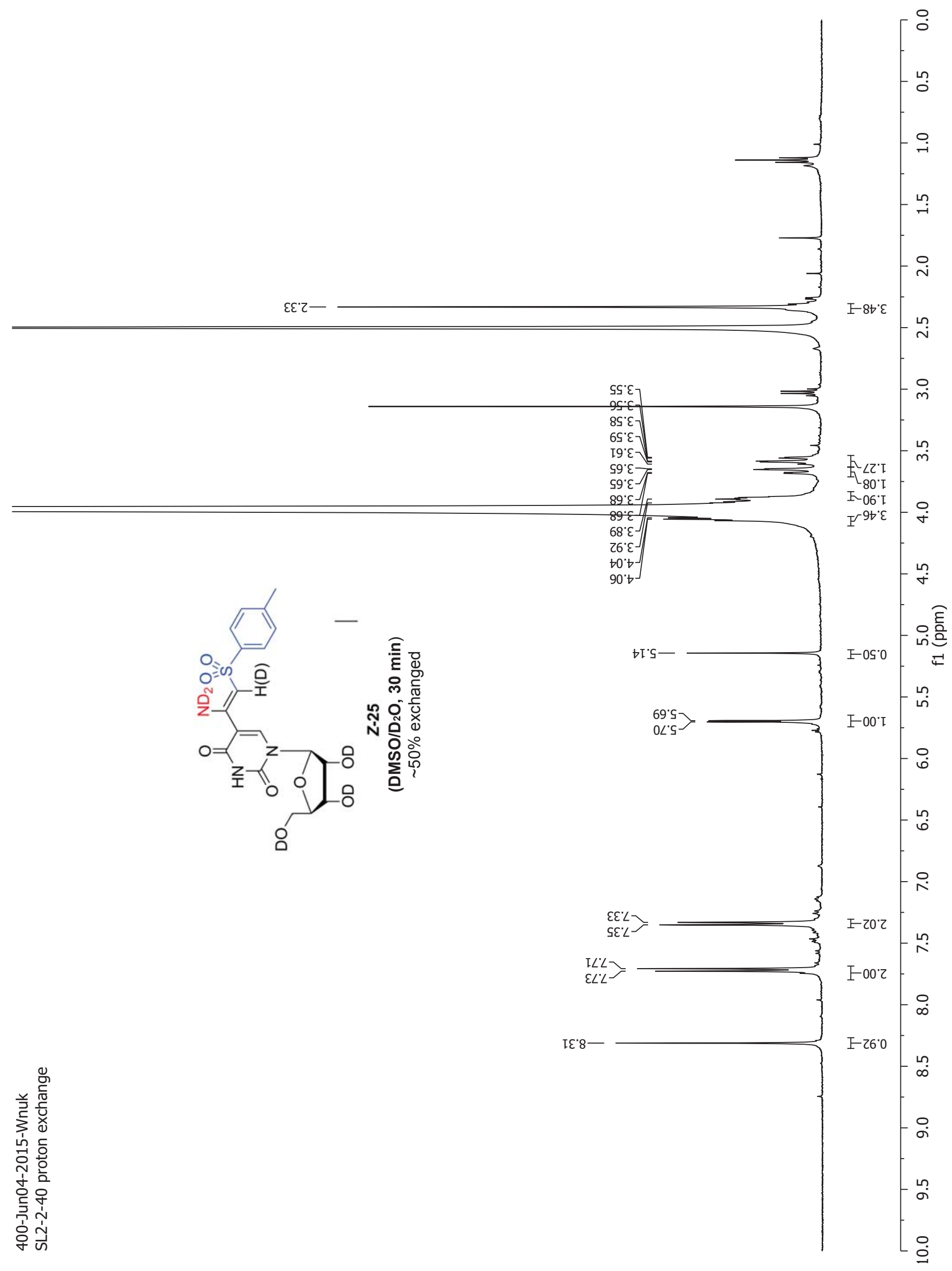


Page |S58

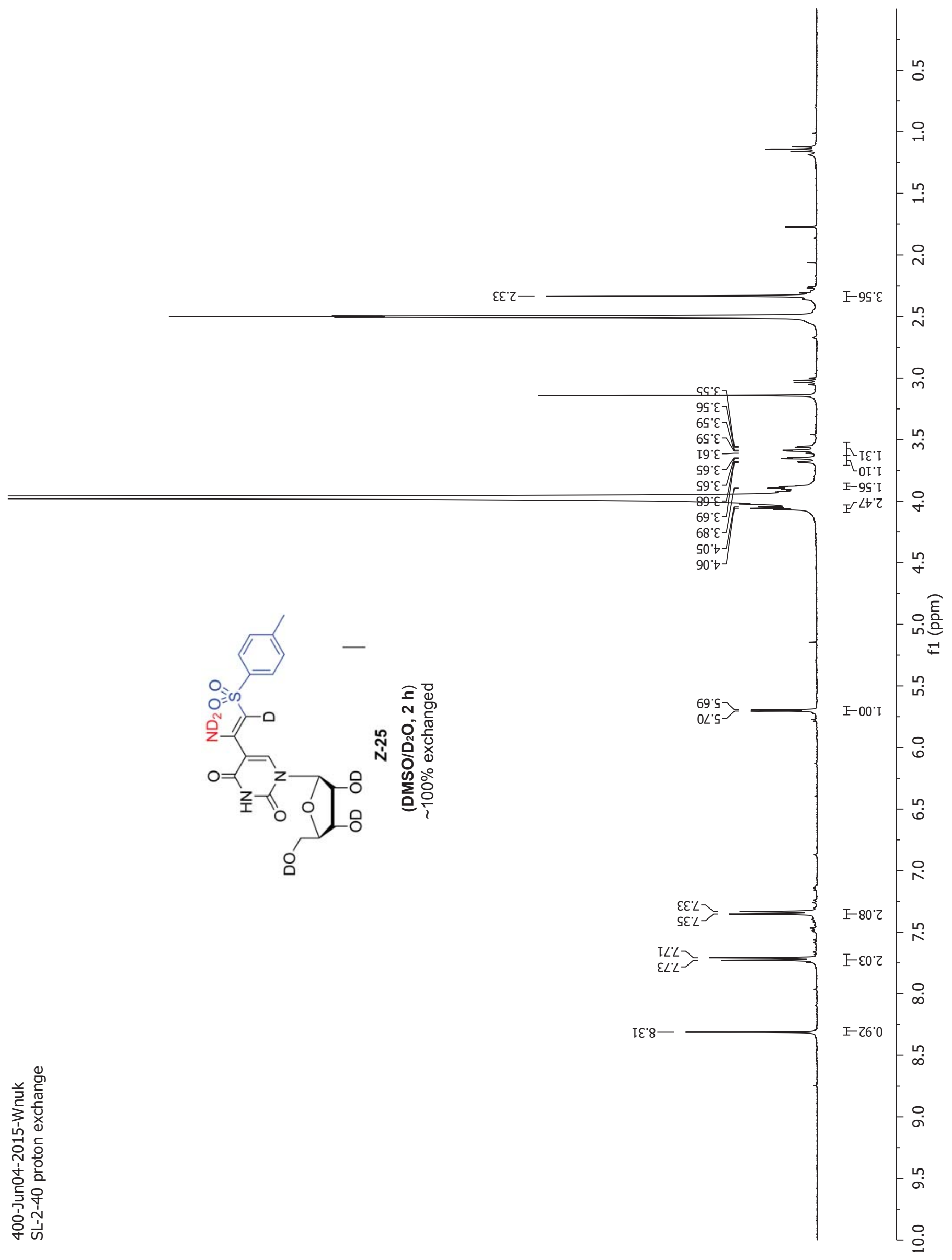


Page |S59

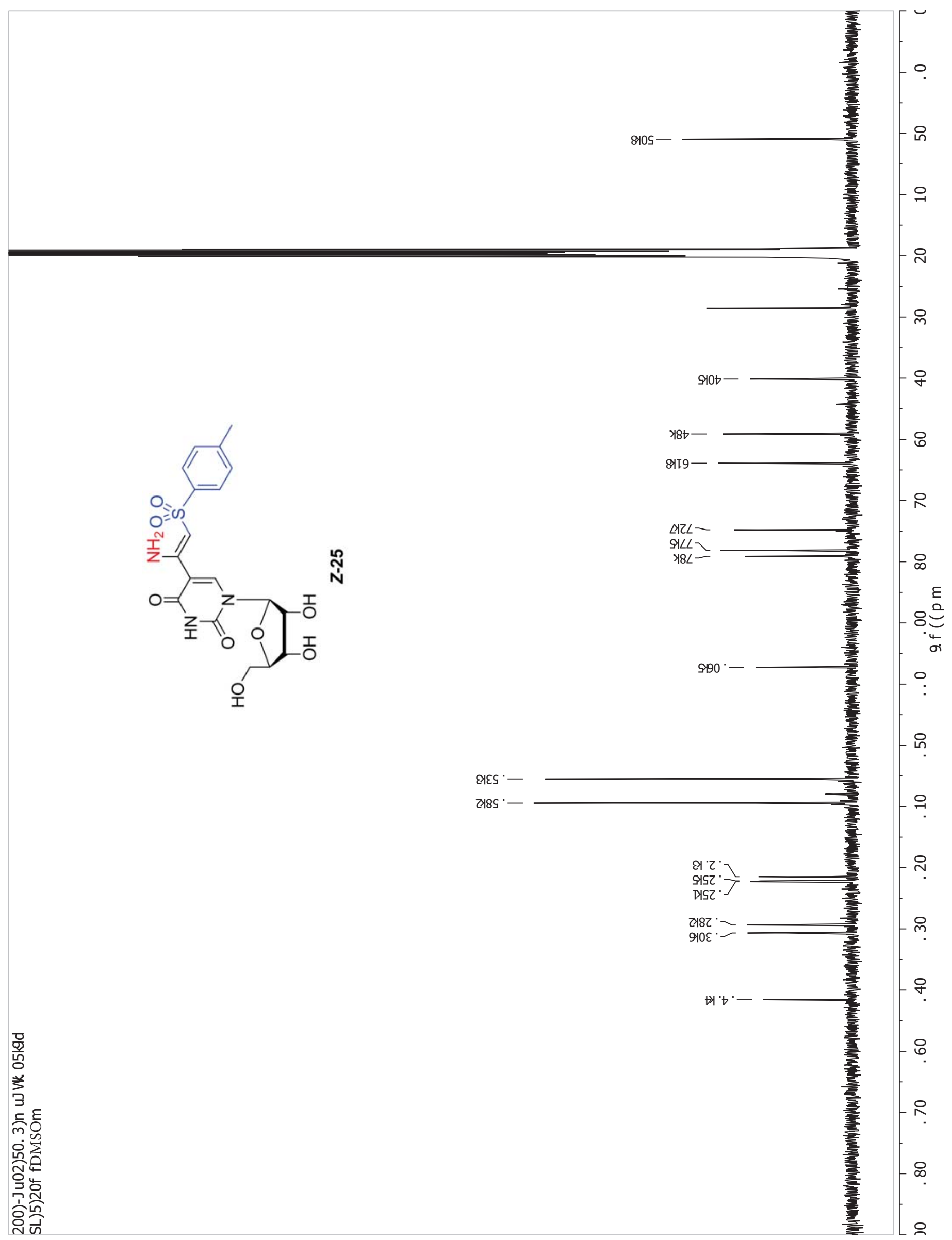


Page |S60

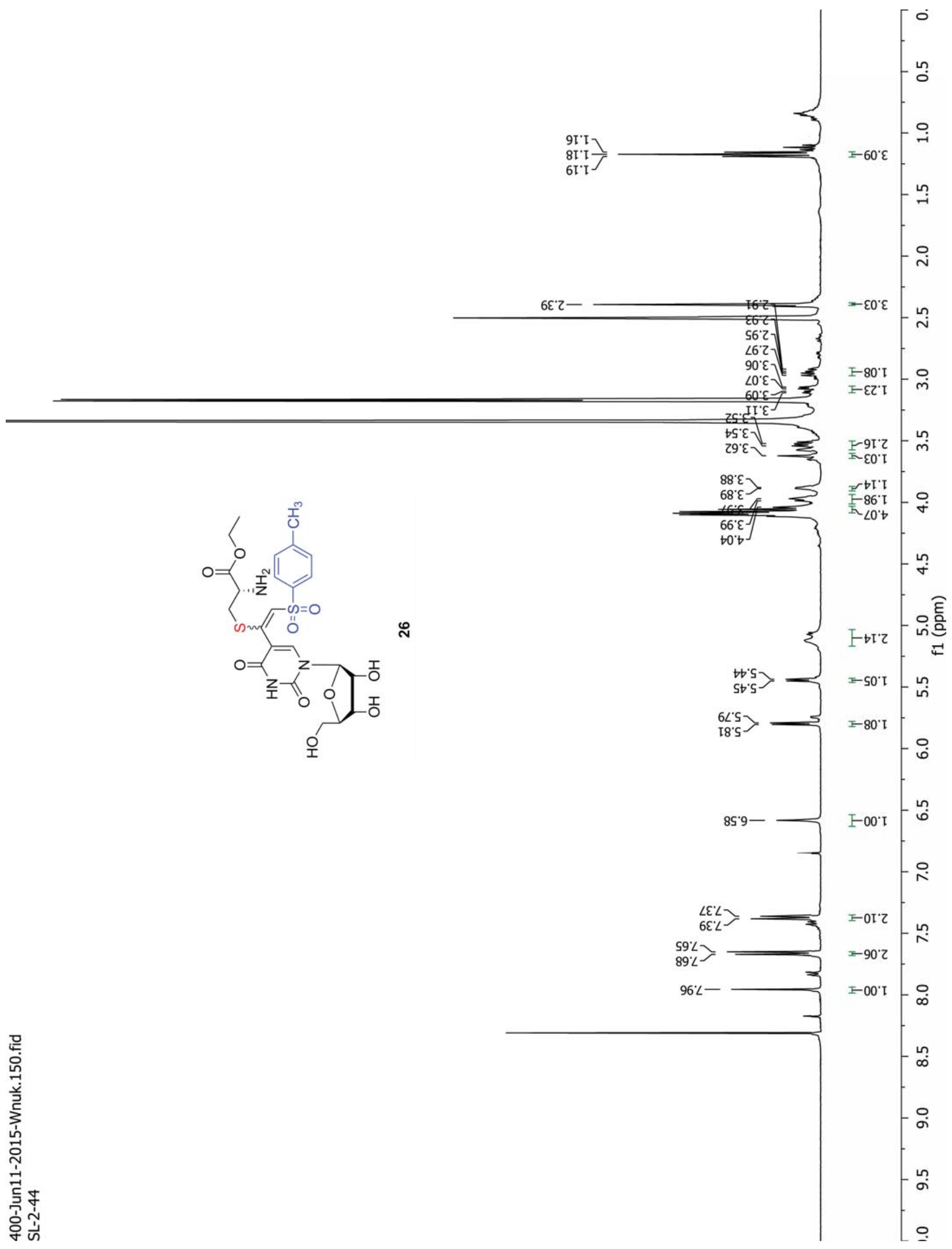


Page |S61

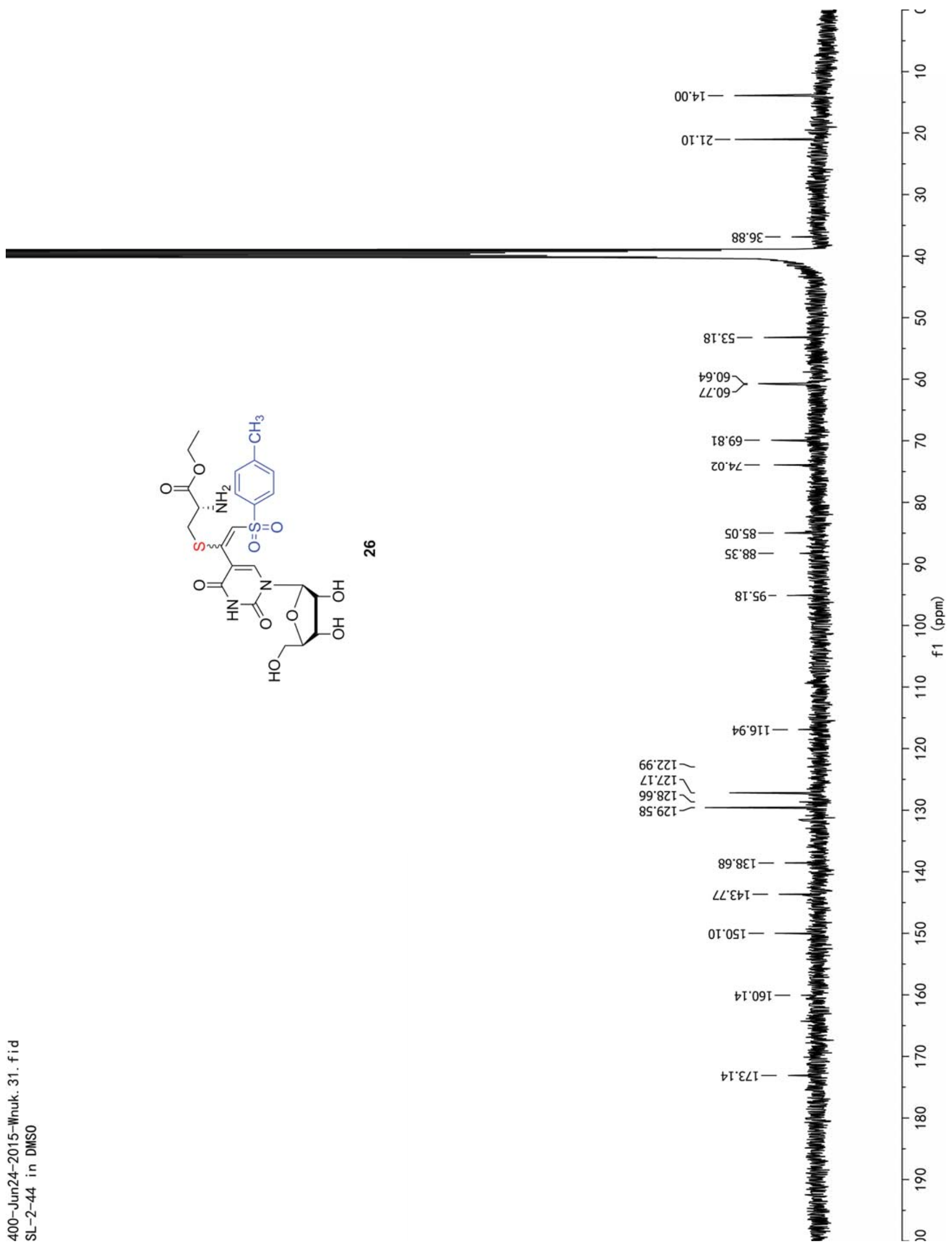


Page |S62

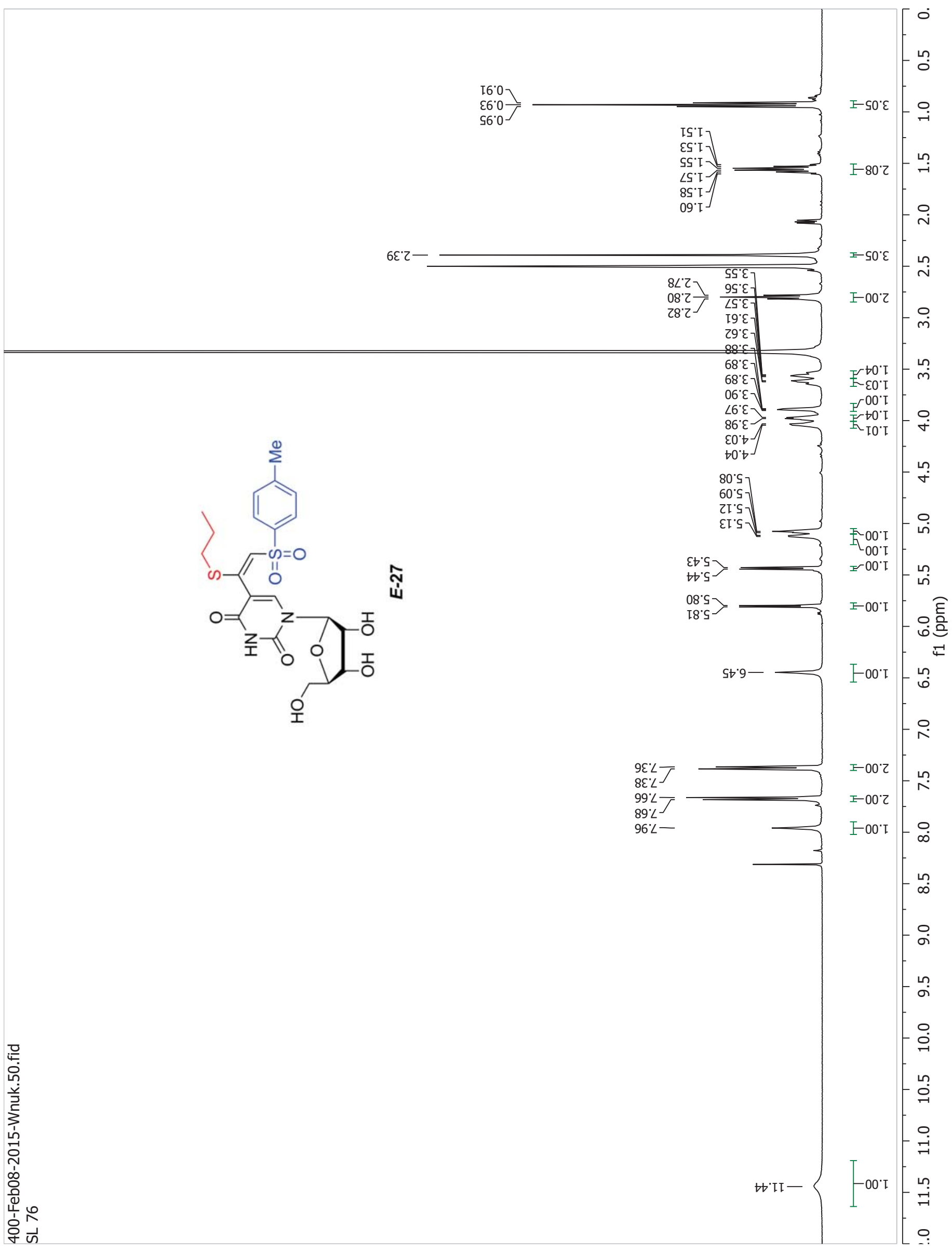


Page |S63

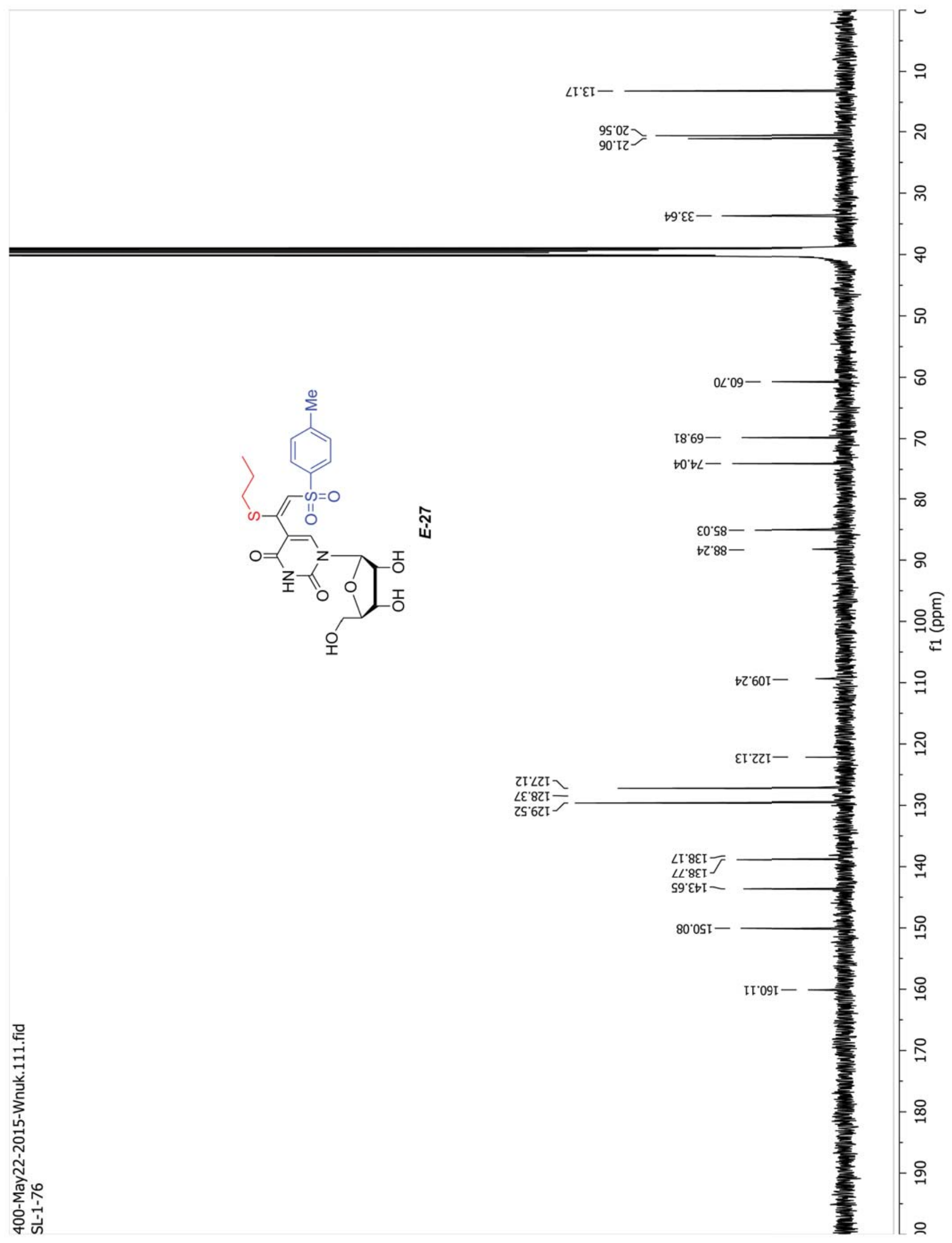


Page |S64

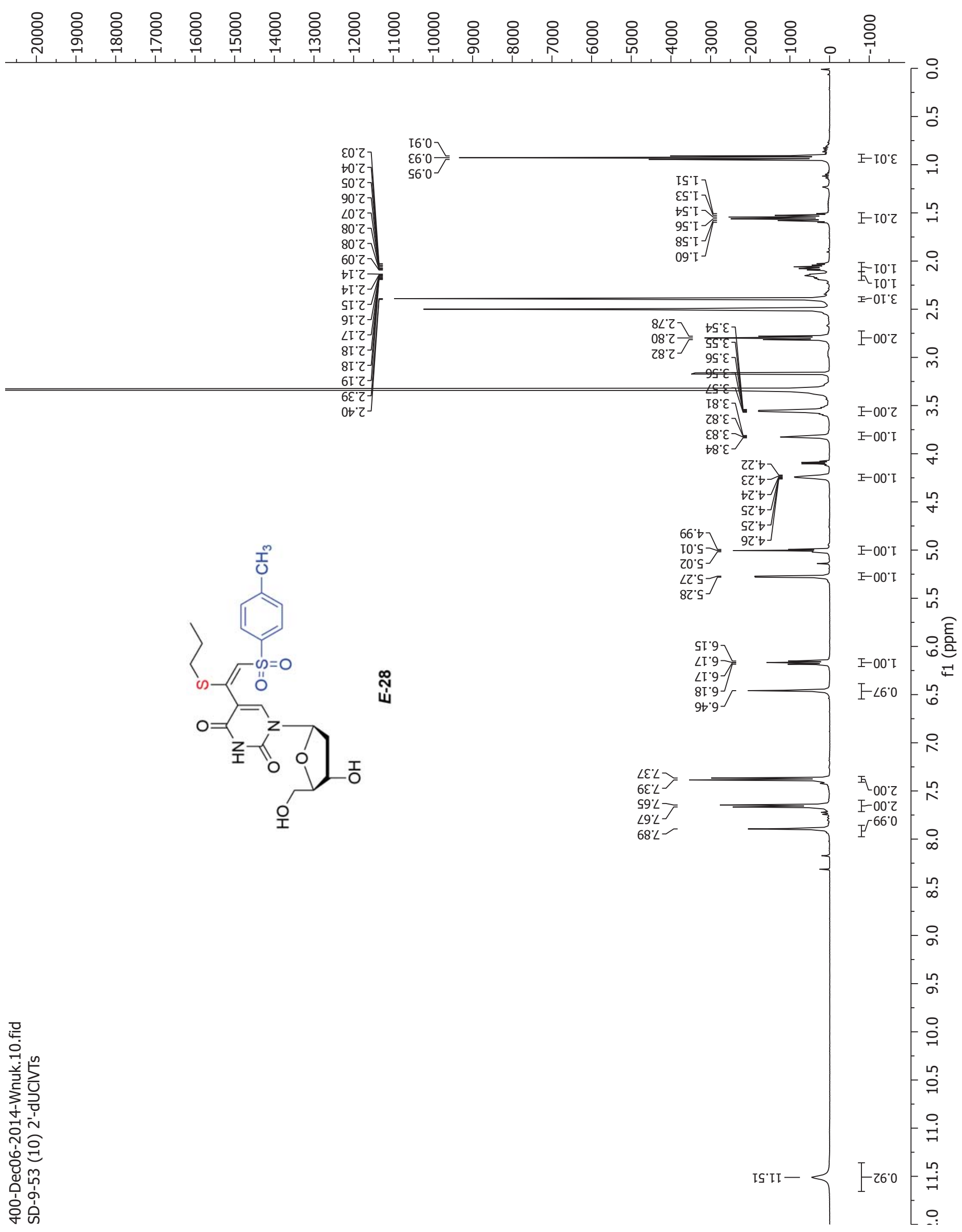


Page |S65

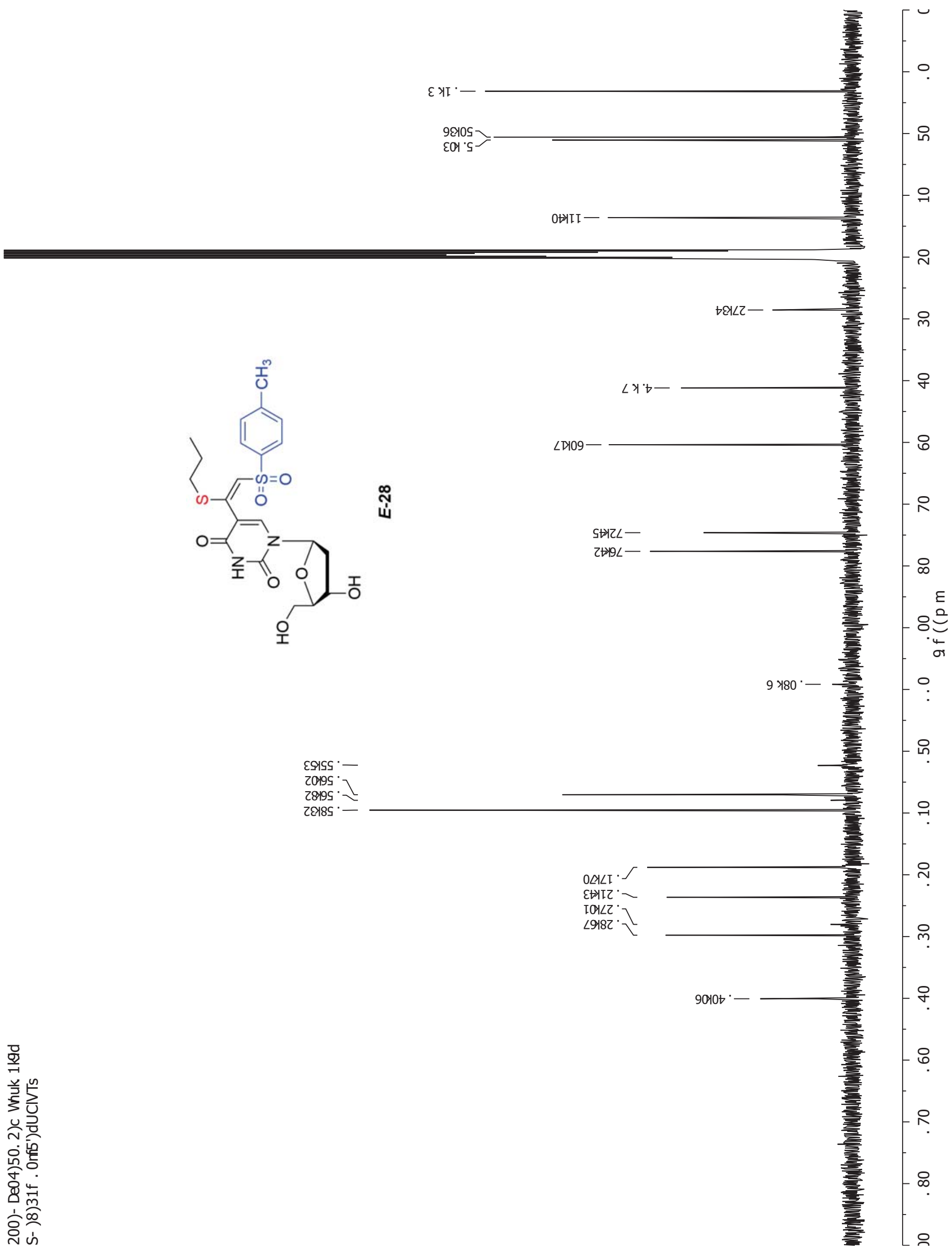


Page |S66

(mdd) If

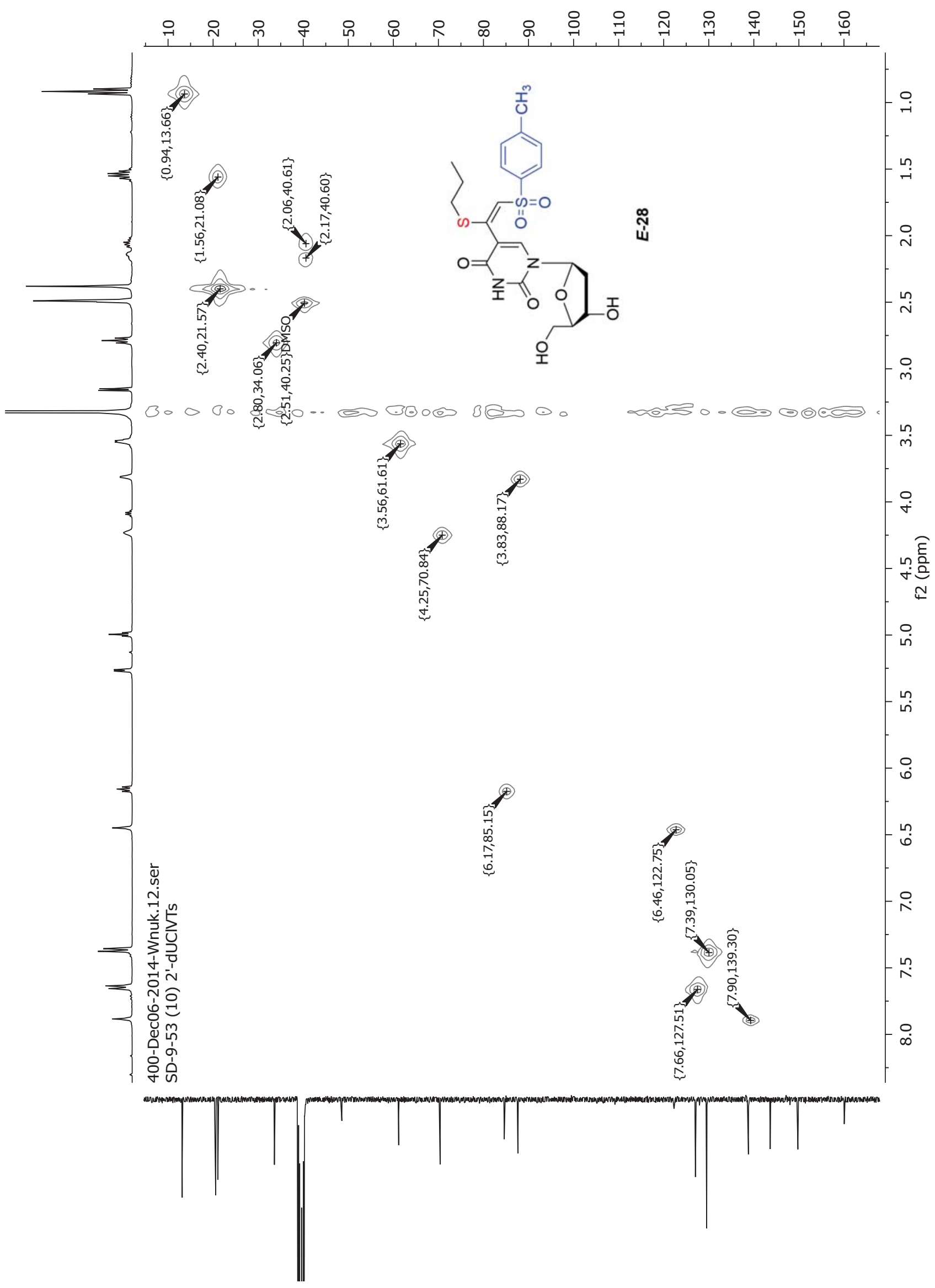


Page |S67

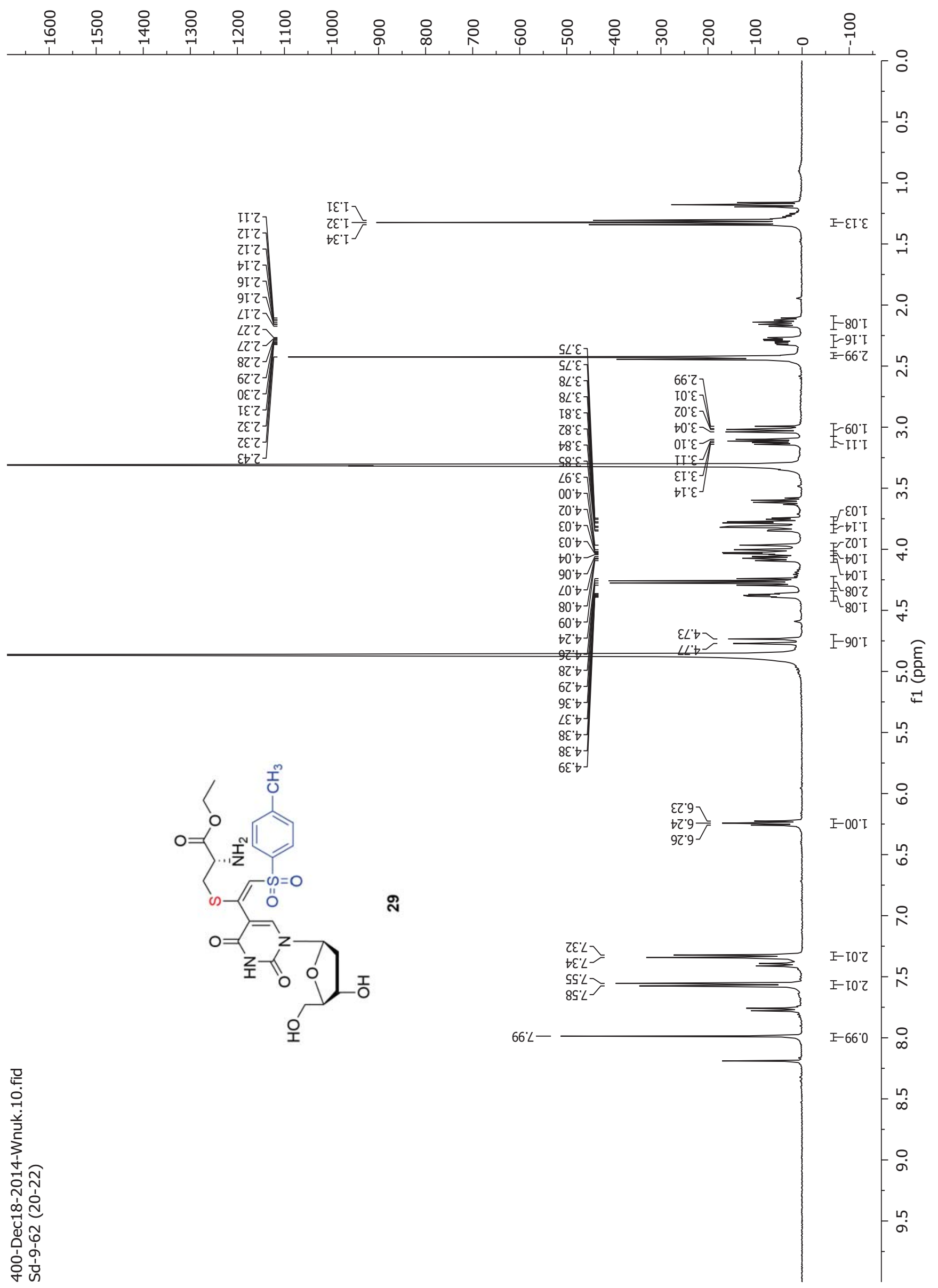


Page |S68

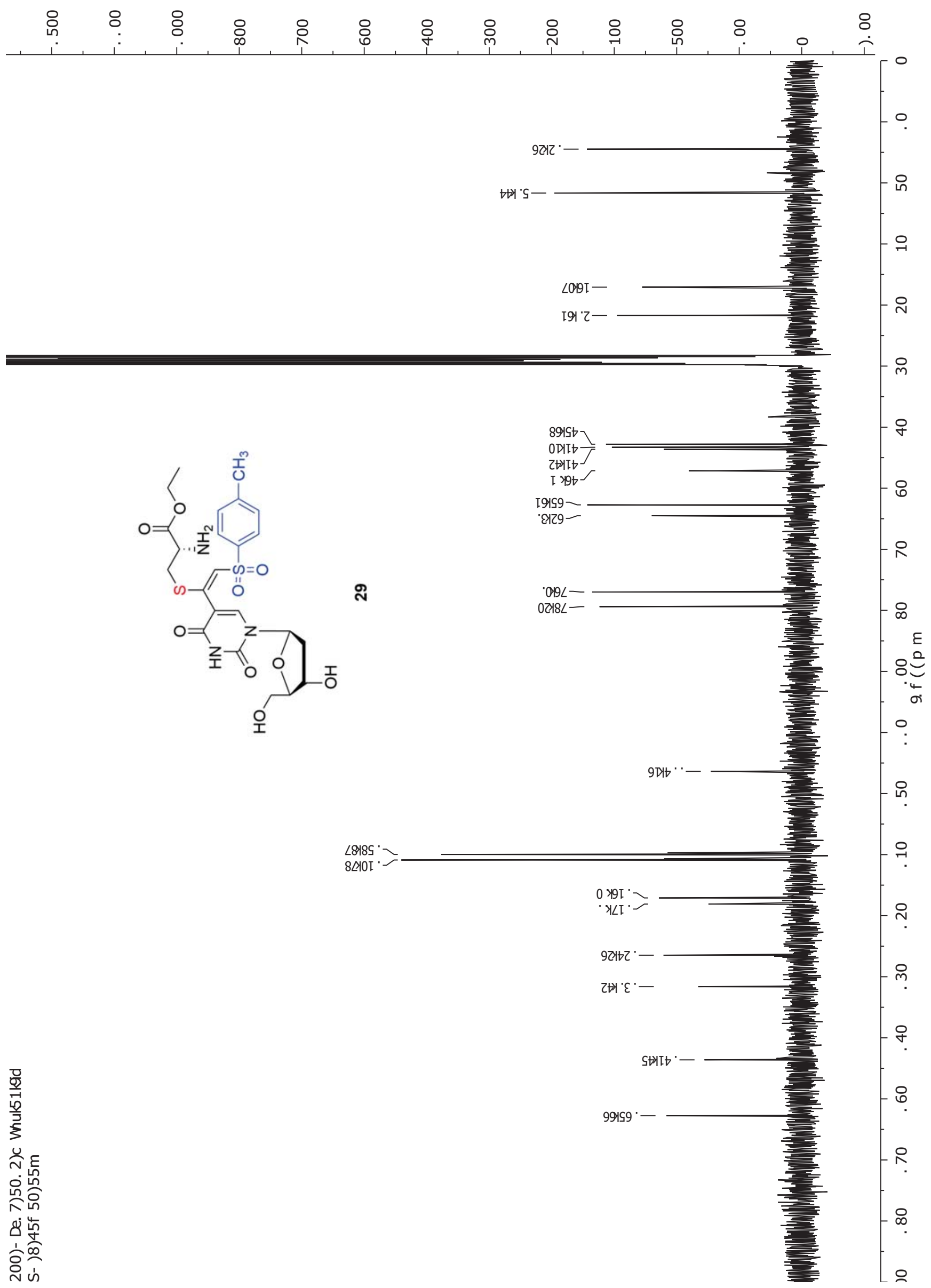

